\title{
IMPLEMENTATION AND VALIDATION OF THE $\zeta-f$ AND ASBM TURBULENCE MODELS
}

\author{
A Thesis \\ Presented to \\ the Faculty of California Polytechnic State University, \\ San Luis Obispo
}

\author{
In Partial Fulfillment \\ of the Requirements for the Degree of \\ Master of Science in Aerospace Engineering
}

by

Dustin Van Blaricom Quint

November 2011 
(C) 2011

Dustin Van Blaricom Quint

ALL RIGHTS RESERVED 
TITLE:

AUTHOR:

DATE SUBMITTED:

COMMITTEE CHAIR:

COMMITTEE MEMBER:

COMMITTEE MEMBER:

COMMITTEE MEMBER:
Implementation and validation of the $\zeta-f$ and ASBM turbulence models.

Dustin Van Blaricom Quint

November 2011

Dr. David Marshall

Dr. Kim Shollenberger

Dr. Jin Tso

Dr. William Durgin 


\section{ABSTRACT \\ Implementation and Validation of the $\zeta-f$ and ASBM Turbulence Models Dustin Van Blaricom Quint}

The use of Computational Fluid Dynamics (CFD) tools throughout the engineering industry has become standard. Simulations are used during nearly all steps throughout the life cycle of products including design, production, and testing. Due to their wide range of use, industrial CFD codes are becoming more flexible and easier to use. These commercial codes require robustness, reliability, and efficiency. Consequently, linear eddy viscosity models (LEVM) are used to model turbulence for an increasing number of flow types. LEVM such as $k-\varepsilon$ and $k-\omega$ provide modeling with little loss of computational efficiency and have proven to be robust. The LEVM that are most common in CFD tools, however, are not adequate for accurate prediction of complex flows. This includes flows with high streamline curvature, strong rotation and separation regions. Unfortunately, due to their ease of use in the commercial CFD tools, the models are used frequently for complex flows. Modifications have been made to LEVM such as $k-\varepsilon$ in order to improve modeling, but generally, the modifications have only improved modeling of less complex flows. More advanced LEVM models have been developed using elliptic relaxation equations to help resolve these issues.

The $v^{2}-f$ model was developed to better capture flow physics for complex flows while being applicable to general flows. It is generally considered one of the most accurate LEVMs. It does, however, have issues with stability and robustness. Several improvements have been proposed. One of the most notable is its reformulation into the 
$\zeta-f$ model which offers several improvements while maintaining accurate flow prediction. The model improvement is still limited by being a LEVM. While models, such as differential Reynolds stress models, do exist which are able to capture relevant flow physics in complex flows, modeling difficulties make them impractical for use in a commercial CFD code.

Algebraic Reynolds stress models have attempted to bridge this gap with varying levels of success. The models express the Reynolds stress tensor as a function of different higher level tensors. This is the same process used to derive non-linear eddyviscosity models which add extra high-order terms to the Boussinesq approximation. According to Kassinos and Reynolds, however, this technique is fundamentally flawed. These models fail to capture all relevant information about the turbulence structure. The Reynolds stresses capture information regarding the turbulent componentiality, i.e. velocity components of turbulence. The dimensionality, which carries information regarding the direction of turbulent eddies, is not modeled, however. Kassinos and Reynolds constructed a structure-based model which attempts to capture turbulent componentiality and dimensionality by expressing the Reynolds stress tensor as a function of one-point turbulence structure tensors. Their original model introduced hypothetical turbulence eddies which could be averaged and then used to relate the eddyaxis transport equation to the proper structure tensors. The ideas behind this model were adapted into several different models including the R-D model and the Q-model. These formulations were able to accurately capture the flow physics for many complex flow types especially those with mean rotation. These resulting models, however, were overly 
complicated for application in commercial CFD codes. These structure-based models later resulted in the development of the algebraic structure based model (ASBM).

The ASBM was developed in order to ensure computational efficiency while capturing relevant turbulence physics. The ASBM uses an algebraic model for the eddy statistics which is constructed from the local mean deformation and two turbulent scales. The original turbulent scales used were the turbulent kinetic energy and the large scale vorticity. Although the model was calibrated specifically for use with the turbulent kinetic energy and large scale vorticity transport equations, the algebraic model can be used in conjunction with any scalar transport equations as long as the field distribution of turbulent kinetic energy and turbulence time scale can be obtained. Based on its formulation, the ASBM, used in combination with any scalar transport equations, should be applicable to most commercial CFD codes.

The objective of this work was to implement the $\zeta_{-} f$ model and ASBM, coupled with $k-\varepsilon$ and $v^{2}-f$, in the commercial CFD solver FLUENT and validate its performance for canonical turbulent flows including a subsonic turbulent flat-plate, S3H4 2D hill, and backward-facing step. Each turbulent flow was evaluated using various turbulence models including Spalart-Allmaras, $k-\varepsilon, k-\omega, k-\omega-\mathrm{SST}, v^{2}-f, \zeta-f$ and two ASBM formulations and compared against experimental results. The $\zeta-f$ model produced improved results for both the flat plate and backward facing step as compared to all twoequation or less turbulence models and showed similar predictive capabilities to the $v^{2}-f$ model. It had difficulties predicting attached flow past the $\mathrm{S} 3 \mathrm{H} 42 \mathrm{D}$ hill just as the $v^{2}-f$ model. This, however, was expected due to its basis on the $v^{2}-f$ model. The model was also more stable than the $v^{2}-f$ model during calculation of the turbulent flat plate but 
showed no improvement in robustness for the more complex backward facing step. The semicoupled (linear eddy viscosity model based) $v^{2}$-f-ASBM's predictive capabilities were comparable to the two equation models for the turbulent flat plate case. It performed surprisingly well for the backward facing step and matched the experimental data within experimental uncertainty. The model did, however, have problems predicting the S3H4 2D hill just as the with the $v^{2}-f$ model.

Keywords: Turbulence Modeling, ASBM, Validation, $\zeta-f$, FLUENT 


\section{ACKNOWLEDGEMENTS}

This work was funded as a part of a NASA research announcement award under Contract \# NNL07AA55C with Craig Hange and Clif Horne as technical monitors.

This work is a part of a NASA research announcement to develop predictive capabilities for an $\mathrm{N}+2$ cruise efficient short take-off and landing (CESTOL) aircraft. The advanced model for extreme lift and improved aeroacoustics (AMELIA), shown below in Figure 1, is the basis for this effort and was designed under a current research program, led by California Polytechnic State University in San Luis Obispo, funded under NASA’s Subsonic Fixed Wing Program.

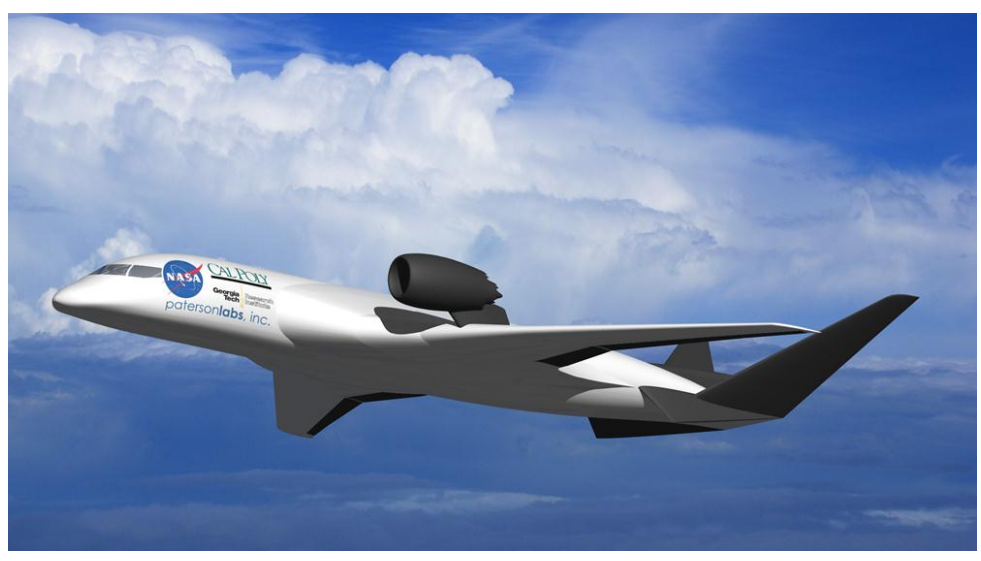

Figure 1. AMELIA, $\mathrm{N}+2$ generation CESTOL aircraft for use in Cal Poly's future wind tunnel test. 


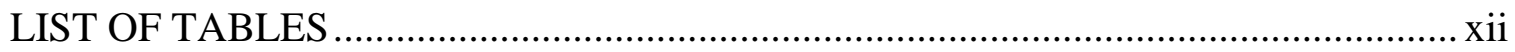

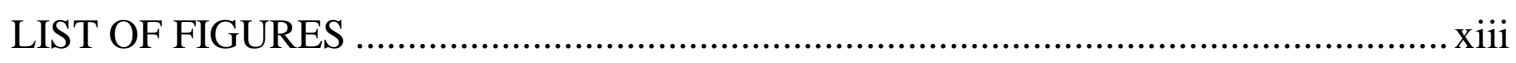

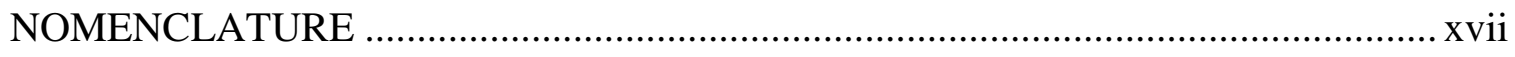

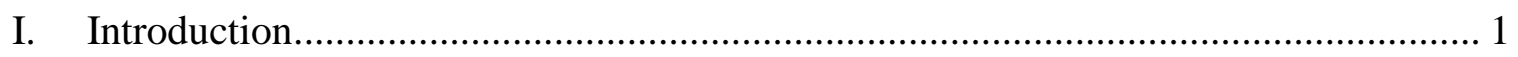

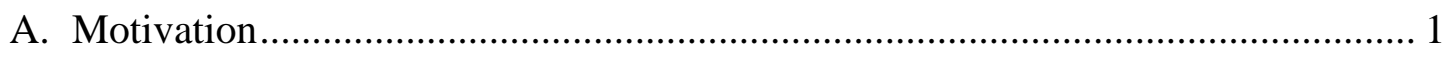

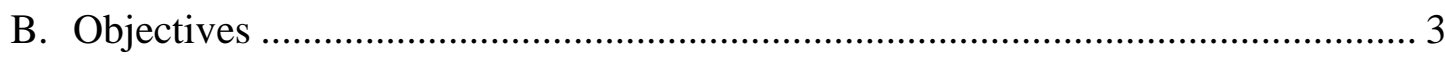

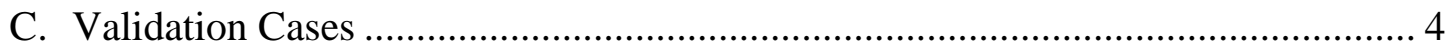

1. Turbulent Flat Plate.................................................................................... 4

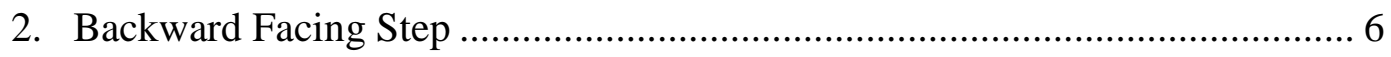

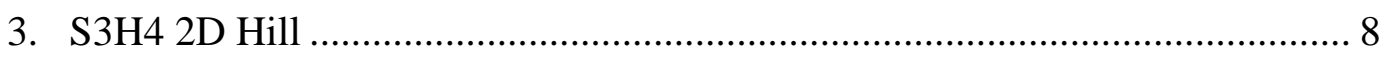

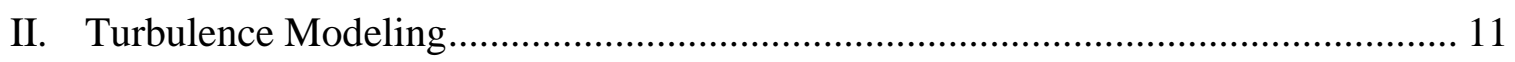

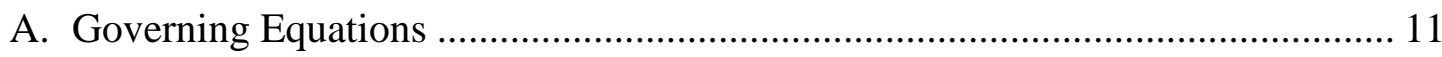

B. Common Turbulence Models ............................................................................ 15

1. Spalart-Allmaras Model........................................................................... 15

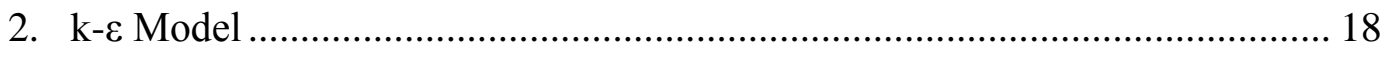

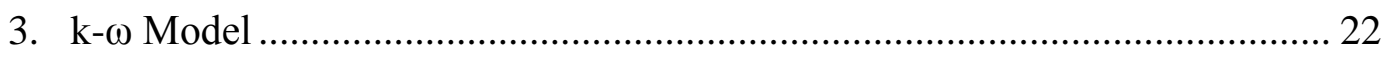

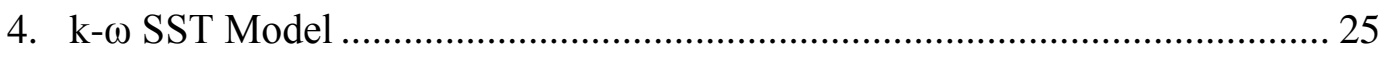

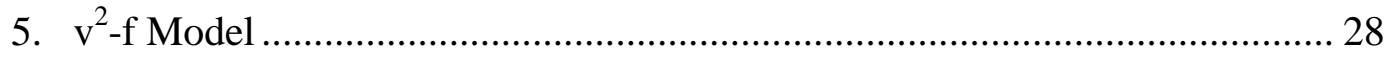

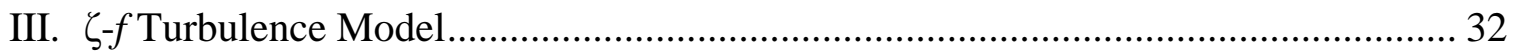

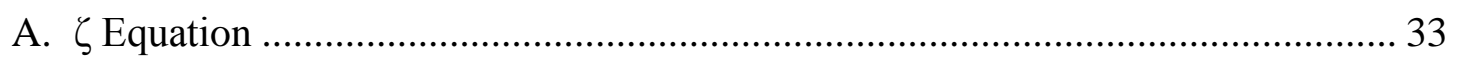

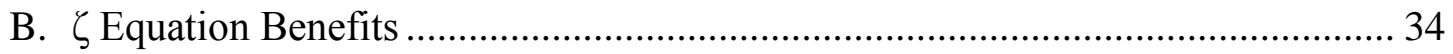


C. Elliptic Relaxation Function .......................................................................... 37

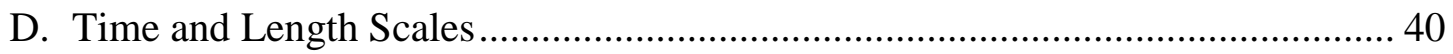

E. $\zeta-f$ with Zero Wall Boundary Condition .................................................................. 41

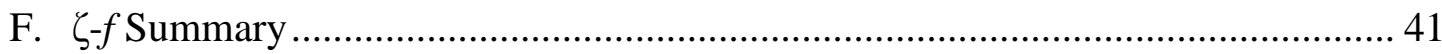

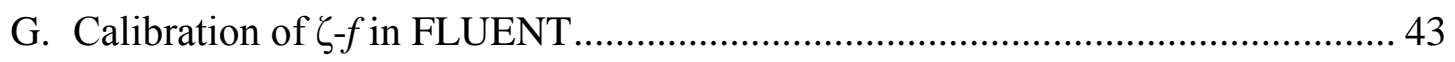

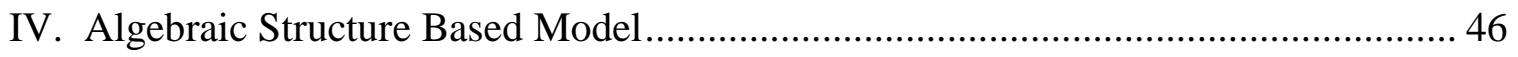

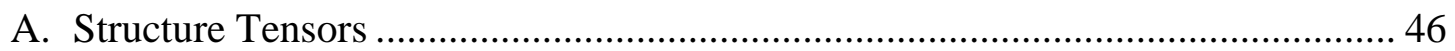

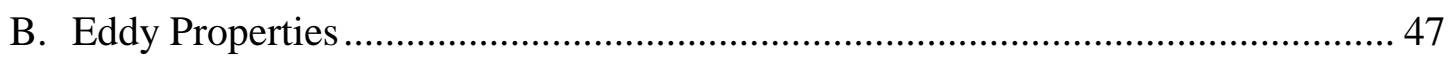

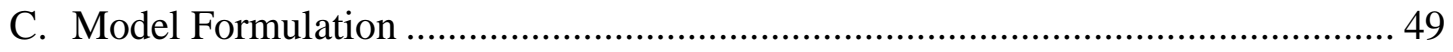

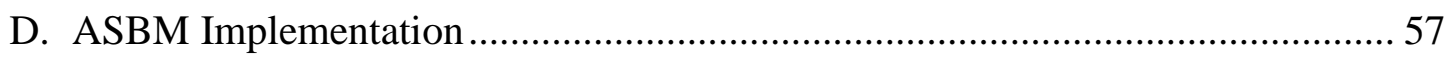

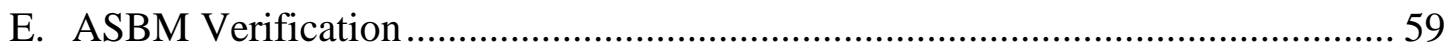

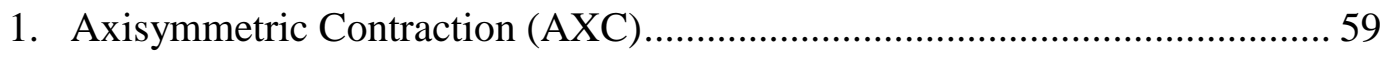

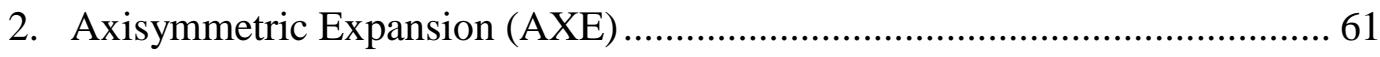

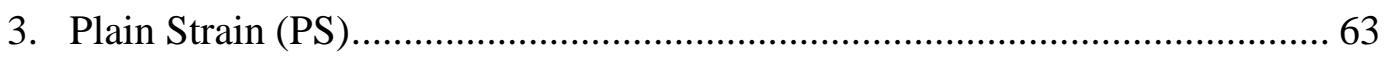

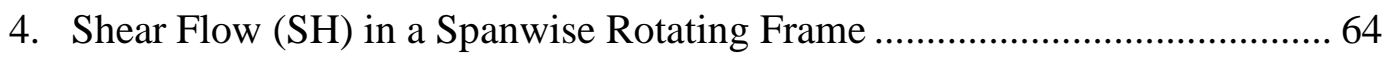

5. Shear Flow (SH) in a Streamwise Rotating Frame ........................................ 66

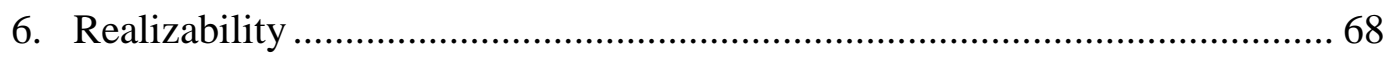

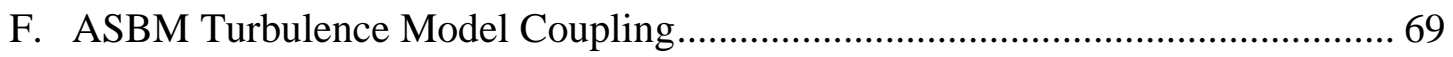

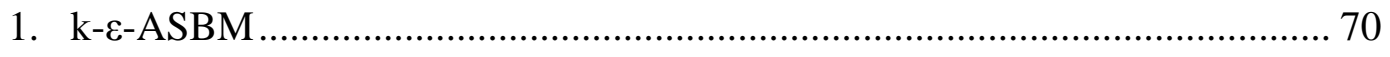

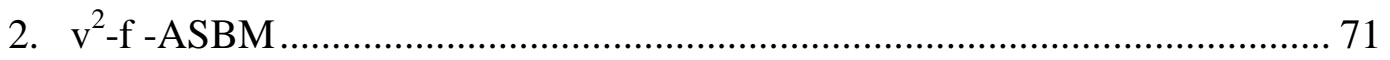

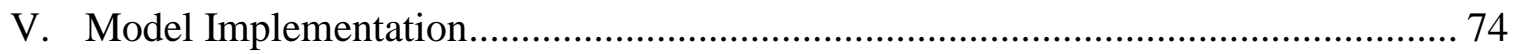

A. User-Defined Fuctions, Scalars, and Memory ………………………................ 74

B. User-Defined Function Architecture..................................................................... 75 


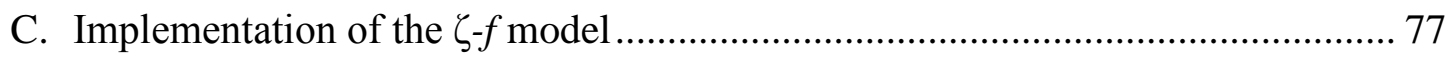

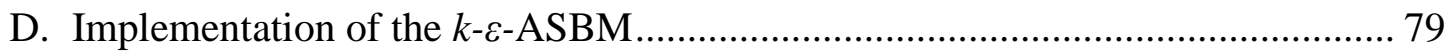

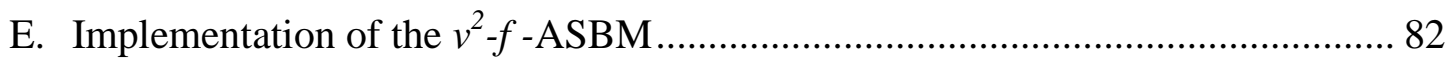

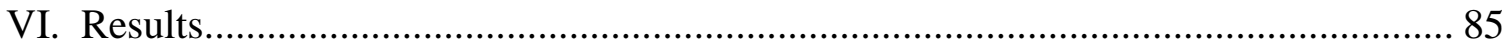

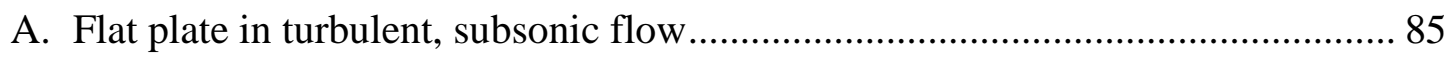

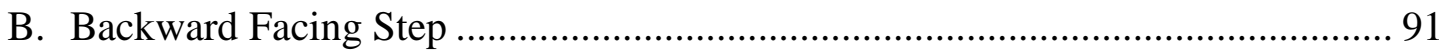

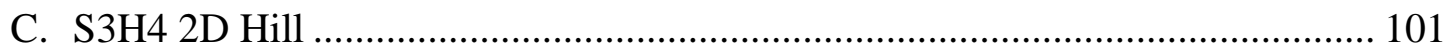

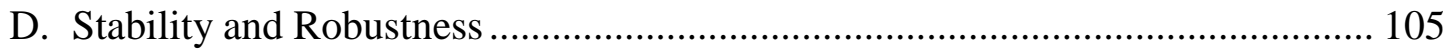

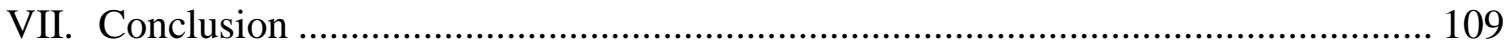

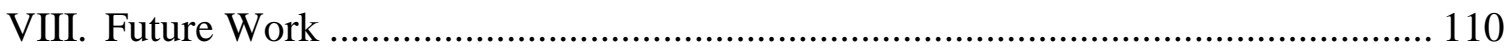

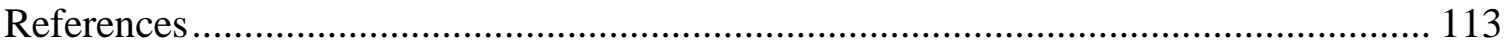




\section{LIST OF TABLES}

Table 1. Summary of $\zeta-f$ model constants determined by Popovac................................ 42

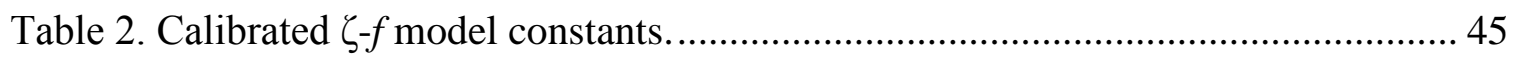

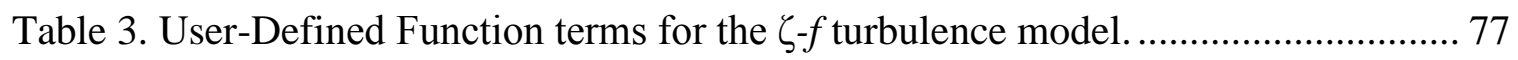

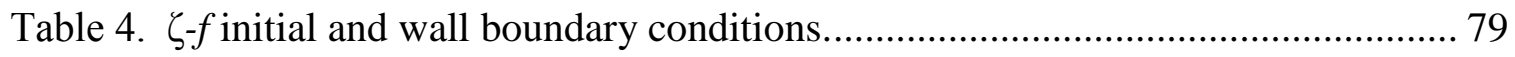

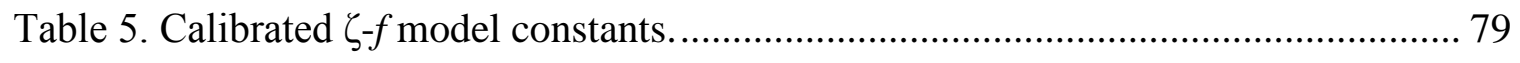

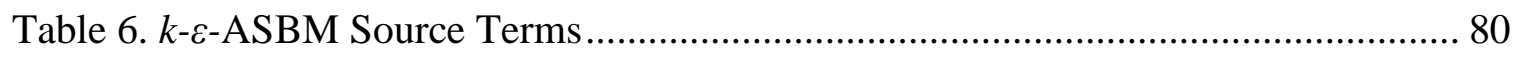

Table 7. Blockage parameter user-defined scalar definition. .................................... 80

Table 8. Velocity source for mean flow coupling................................................... 81

Table 9. User-Defined Function terms for the $v^{2}-f$-ASBM turbulence model................ 82

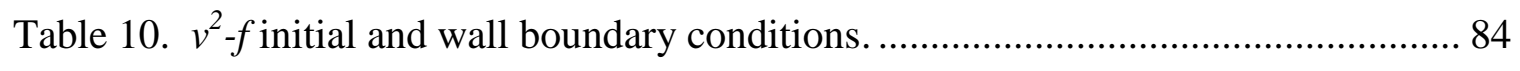

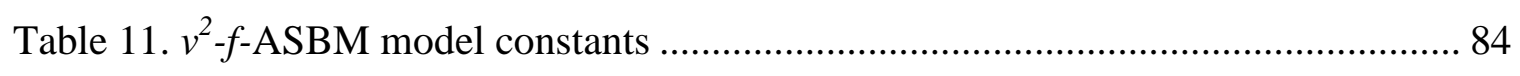

Table 12. Comparison of reattachment length to experimental data. ............................. 94 


\section{LIST OF FIGURES}

Figure 1. AMELIA, N+2 generation CESTOL aircraft for use in Cal Poly's future

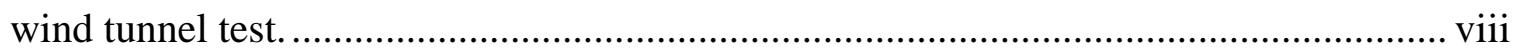

Figure 2. Non-dimensionalized Reynolds stress at $\mathrm{Re}_{\mathrm{x}}=4.2 \mathrm{e} 6$ by Klebanoff...................6

Figure 3. Backward facing step geometry and defining flow characteristics..................

Figure 4. Velocity profiles for a backward facing step by Jovic and Driver. ....................8

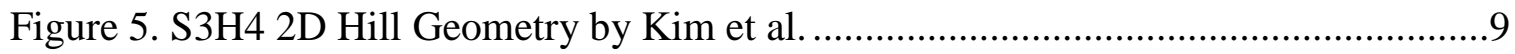

Figure 6. Velocity profiles over the S3H4 hill by Kim et al......................................

Figure 7. Calibration curves for skin friction coefficient. ............................................44

Figure 8. Calibration curves for boundary layer profile. ............................................45

Figure 9. Free shear flow ASBM results under AXC deformation with no frame rotation $\Omega^{f}=0$ (solid line), $\Omega^{f}=S$ (dashed line), $\Omega^{f}=2 S$ (dashed-dot line)

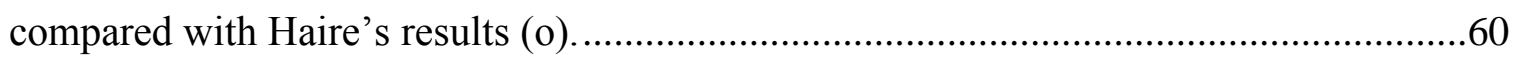

Figure 10. Wall bounded ASBM results under AXC deformation with no frame rotation $\Omega^{f}=0$ (solid line), and $\Omega^{f}=2 \Gamma$ (dashed-dot line) compared with Langer's

results (o).

Figure 11. Free shear flow ASBM results under AXE deformation with no frame rotation $\Omega^{f}=0$ (solid line), $\Omega^{f}=S$ (dashed line), $\Omega^{f}=2 S$ (dashed-dot line) compared with Haire's results (o).

Figure 12. Wall bounded ASBM results under AXE deformation with no frame rotation $\Omega^{f}=0$ (solid line), and $\Omega^{f}=2 \Gamma$ (dashed-dot line) compared with Langer's results (o) 
Figure 13. Free shear flow ASBM results under PS deformation with no frame rotation $\Omega^{f}=0$ (solid line), $\Omega^{f}=S$ (dashed line), $\Omega^{f}=2 S$ (dashed-dot line) compared with Haire's results (o)

Figure 14. Free shear flow ASBM results under $\mathrm{SH}$ with spanwise rotation deformation with no frame rotation $\Omega^{f}=0$ (a), $\Omega_{3}^{f} / S=0.1(\mathrm{~b}), \Omega_{3}^{f} / S=0.5$ (c), and $\Omega_{3}^{f} / S=1.0(\mathrm{~d})$ compared with Haire's results $(\mathrm{o})$.

Figure 15. Wall bounded ASBM results under $\mathrm{SH}$ with spanwise rotation deformation with no frame rotation $\eta_{f}=0$ (a), $\eta_{f}=0.5(\mathrm{~b}), \eta_{f}=1.0$ (c), $\eta_{f}=1.5$ (d), $\eta_{f}=2.0(\mathrm{e})$, and $\eta_{f}=-2.0(\mathrm{f})$ compared with Langer's results (o).

Figure 16. Wall bounded ASBM results under $\mathrm{SH}$ with streamwise rotation deformation with no frame rotation $\Omega_{23}^{f} / \Omega_{12}=0$ (solid line), and $\Omega_{23}^{f} / \Omega_{12}=-0.5$ (dashed-dot line) compared with Langer's results (o).

Figure 17. Anisotropy Invariant map for AXC (a), AXE (b), PS (c), and SH (d).

Figure 18. Architecture for user access to the FLUENT solver. .76

Figure 19. Skin fiction coefficient distribution for a turbulent flat plate in subsonic

flow.

Figure 20. Boundary layer velocity profiles of a turbulent flat plate produced by various turbulence models compared with experimental data. .87

Figure 21 . Boundary layer velocity profiles of a turbulent flat plate produced by $\zeta$ $f$ and $v^{2}-f$-ASBM compared with experimental data. .88

Figure 22. Reynolds stresses at $\operatorname{Re}_{\mathrm{x}}=4.2 \times 10^{6}$ using the Boussinesq approximation and ASBM compared against experimental data of Klebanoff. 
Figure 23. Reynolds stresses at $\operatorname{Re}_{\mathrm{x}}=4.2 \times 10^{6}$ using the $v^{2}-f$-ASBM compared

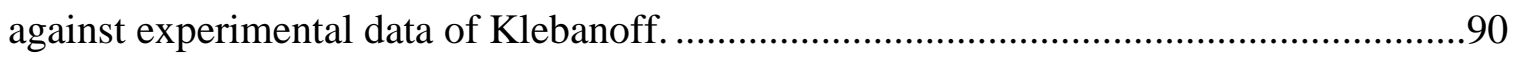

Figure 24. Separation region for the $k-\varepsilon$ model (a), and the $k-\omega$ model (b)...................92

Figure 25. Separation region for the $k$ - $\omega$-SST model (a), and the $v^{2}-f$ model (b)............93

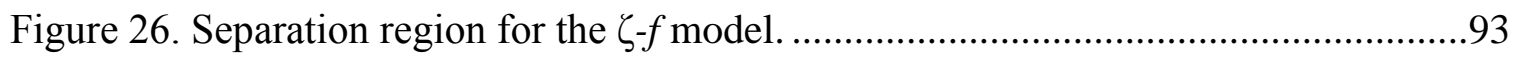

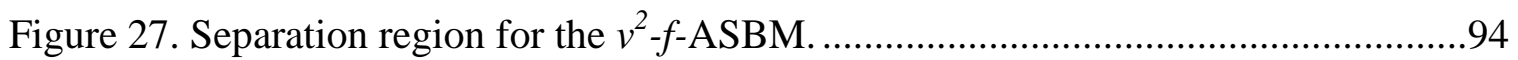

Figure 28. Streamwise velocity profiles at $x / h=-3,4,6,10,15$, and 19 for $v^{2}-f$ (solid line) compared against experimental data ( $\square)$.

Figure 29. Streamwise velocity profiles at $x / h=-3,4,6,10,15$, and 19 for $\zeta-f$ (solid line) compared against experimental data $(\square)$.

Figure 30. Streamwise velocity profiles at $x / h=-3,4,6,10,15$, and 19 for $v^{2}-f$ ASBM (solid line) compared against experimental data ( $\square)$.

Figure 31. Backward facing step Reynolds stresses determined by the Boussinesq approximation with the $v^{2}-f$ model (solid lines) compared against experimental data $(\square)$

Figure 32. Backward facing step Reynolds stresses determined by the ASBM with the $v^{2}-f$ model (solid lines) compared against experimental data ( $\square$ ).

Figure 33. Backward facing step Reynolds stresses determined by the $v^{2}-f$-ASBM model (solid lines) compared against experimental data ( $\square$ ).

Figure 34. S3H4 2D hill velocity profiles for various turbulence models (solid line) compared against experimental data ( $\square)$.

Figure 35. S3H4 2D hill velocity profiles for the $\zeta-f$ model (solid line) compared against experimental data $(\square)$ .104 
Figure 36. S3H4 2D hill velocity profiles for the $v^{2}-f$-ASBM (solid line) compared

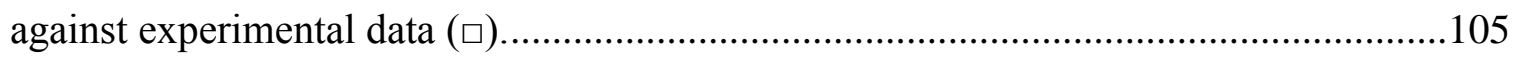

Figure 37. Residuals of $v^{2}-f$ model for turbulent flat plate. .......................................106

Figure 38. Residuals of $\zeta-f$ model for turbulent flat plate .........................................106

Figure 39. Boundary layer velocity profiles of a turbulent flat plate produced by $k$ -

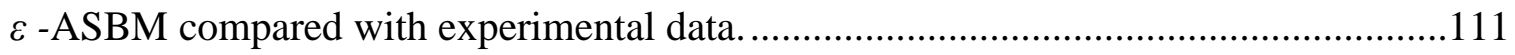

Figure 40. Reynolds stresses at $\operatorname{Re}_{\mathrm{x}}=4.2 \times 10^{6}$ using the fully coupled $k-\varepsilon-\mathrm{ASBM}$

compared against experimental data of Klebanoff. 


\section{NOMENCLATURE}

$$
\begin{aligned}
a & =\text { speed of sound } \\
a_{0} & =\text { "slow" constant } \\
a_{i j} & =\text { eddy axis tensor, anisotropy tensor } \\
a_{i j}{ }^{w} & =\text { wall blockage eddy axis tensor } \\
a_{i j}{ }^{*} & =\text { strained eddy axis tensor } \\
B_{i j} & =\text { blockage tensor } \\
b_{i j} & =\text { flattening tensor } \\
C & =\text { model constant } \\
C_{f} & =\text { skin friction coefficient } \\
D_{i j} & =\text { dimensionality structure tensor } \\
L_{1} & =\text { hill half length } \\
d & =\text { wall distance } \\
L_{i j} & =\text { turbulent kinetic energy } \\
f_{i j} & =\text { normalized dimensionality structure tensor } \\
F_{i j} & =\text { circulicity structure tensor } \\
F_{i j}{ }^{r} & =\text { eddy-flattening tensor } \\
f & =\text { hill height }
\end{aligned}
$$




$$
\begin{aligned}
& M_{t}=\text { turbulent Mach number } \\
& P=\text { production } \\
& p=\text { pressure } \\
& q^{2}=\text { twice the turbulent kinetic energy } \\
& R_{i j}=\text { Reynolds stress tensor, pressure rate of strain } \\
& \operatorname{Re}=\text { Reynolds number } \\
& r_{i j}=\text { normalized Reynolds stress tensor } \\
& S=\text { mean strain rate magnitude } \\
& S_{i j}=\text { mean strain rate tensor } \\
& S_{i j}{ }^{*}=\text { traceless strain rate tensor } \\
& T=\text { turbulent time scale } \\
& U=\text { velocity } \\
& u=\text { mean streamwise velocity } \\
& u^{\prime}=\text { fluctuating streamwise velocity } \\
& u^{+}=\text {dimensionless wall velocity } \\
& v=\text { mean wall normal velocity } \\
& v^{\prime}=\text { fluctuating wall normal velocity } \\
& v^{2}=\text { velocity scale } \\
& X=\text { cross diffusion of } \zeta-f \\
& x, y=\text { Cartesian coordinates } \\
& Y=\text { dissipation } \\
& y=\text { wall normal distance } \\
& y^{+}=\text {dimensionless wall distance }
\end{aligned}
$$




$$
\begin{aligned}
& \alpha=\text { damping function } \\
& \gamma_{k}=\text { eddy-helical vector } \\
& \delta=\text { boundary layer height } \\
& \delta_{i j}=\text { Kronecker delta } \\
& \varepsilon=\text { turbulence dissipation rate } \\
& \varepsilon_{i j k}=\text { Levi-Cevita symbol } \\
& \zeta=\text { normalized velocity scale } \\
& \kappa=\text { von Karman constant } \\
& \lambda_{\varepsilon}=\text { blending function } \\
& \mu_{t}=\text { turbulent viscosity } \\
& v=\text { kinematic viscosity } \\
& v^{*}=\text { wall-friction velocity } \\
& \tilde{v}=\text { turbulent eddy viscosity } \\
& \rho=\text { density } \\
& \sigma=\text { turbulent Prandtl number } \\
& \tau=\text { turbulence time scale } \\
& \tau_{w}=\text { wall shear stress } \\
& \Phi=\text { blocking parameter } \\
& \varphi=\text { eddy-jetal parameter } \\
& \chi=\text { eddy-flattening scalar } \\
& \psi=\text { arbitrary instantaneous flow variable } \\
& \Omega=\text { mean rotation rate magnitude } \\
& \Omega_{i}=\text { mean rotation vector }
\end{aligned}
$$


$\Omega_{i j}=$ mean rotation rate tensor

$\Omega_{i}^{f}=$ frame rotation vector

$\Omega_{k}{ }^{\mathrm{T}}=$ total rotation vector

$\omega=$ specific dissipation rate 


\section{Introduction}

\section{A. Motivation}

The computational methods for turbulent flows have been a perplexing problem for over a century. Its mystery is not only due to its lack of general analytic solution, but also the necessity to accurately capture its behavior. Turbulent flow is present in countless engineering applications which require simulation to further improve predictive performance and even initial design. Simulations are used during nearly all steps throughout the life cycle of products including design, production, and testing. Unfortunately, turbulence is very complex and varies greatly depending on application due to the presence of complex spatial and temporal interactions. While the motion of viscous fluids can be solved completely as described by the Navier-Stokes equations, simulation requires extensive computation resources which are out of reach for most applications. Since there are no general analytic solutions for turbulence and full computational solutions are too expensive, models have been developed based on mathematical principles and experimental results.

Modeling is achieved by first simplifying the multitude of both spatial and temporal scales present in turbulent flow. The complex flow is averaged in some way which greatly reduces the number of turbulent scales affecting the mean flow. The averaging of information essentially discards turbulent scale information and their effects are instead expressed through a new term, the Reynolds stress tensor. These Reynolds stresses define the turbulent interaction with the mean flow. Unfortunately, these Reynolds 
stresses are also unknown. The modeling of this tensor is thus the goal of turbulence modeling.

One accepted and widely used technique for modeling the Reynolds stress tensor was proposed over 100 years ago by Boussinesq. This approximation, known as the Boussinesq hypothesis, is based on the assumption that turbulent shearing stresses are related to the rate of mean strain through a scalar turbulent viscosity, commonly referred to as an eddy viscosity. Thus, the eddy viscosity, usually defined analogously with kinetic theory, must be modeled in order to solve the closure problem. Models utilizing this assumption, linear eddy viscosity models (LEVM), are most widely used for engineering purposes due to their simplicity and accuracy for simple flows. Since the assumption does not always hold true, however, LEVM are still limited.

Linear eddy viscosity turbulence model techniques began as seemingly simple relations based on kinetic theory, experimental results and convenience. These simple zero equation models, including mixing length models and Baldwin-Lomax, are applicable for simple flows which are similar to flows in which the models were tailored to represent. Accuracy, however, is sacrificed for simplicity. More computationally expensive models were developed basing the turbulent viscosity on the turbulent kinetic energy, a measurable quantity that is easily interpreted physically. A transport equation for the turbulent kinetic energy can be developed based on the Navier-Stokes equations, cementing its applicability. Multiple models have been developed with this transport equation. These include one equation models utilizing algebraic relations to model unknown terms, two equation models which introduce a second transport equation to model unknowns, and more. Generally, with increasing number of equations, the model 
becomes more versatile. One of the most versatile and well used LEVM is the $v^{2}-f$ model. It seems though, that with better predictive capabilities come sacrifices in robustness and ease of use. What is truly desired then is a model which offers robust predictive capabilities on a myriad of flows. Popovac has attempted this through the modification and reformulation of a normalized $v^{2}-f$ model.

While LEVMs are the most widely used in industry, constant sensitization to varying flow types, combined with the use of the Boussinesq hypothesis, are deterrents for use in increasingly complex flows. More complex models which abandon the linear eddy viscosity approach model each of the Reynolds stresses individually. These Reynolds stress models, while accounting for more complex flow phenomena, are computationally expensive requiring an additional five transport equations for two dimensional flow. The goal then is to develop a model which captures relevant flow characteristics, models the Reynolds stresses accurately, and remains relatively inexpensive computationally. Kassinos, Reynolds, and coworkers have been developing a model which will capture relevant flow characteristics while maintaining computational efficiency.

\section{B. Objectives}

In order to improve the predictive capabilities and robustness of CFD, new turbulence models are formulated and tested regularly. It is difficult, however, for newly developed models to become widespread due to their limited application to commercial CFD solvers. While predictive capability is important, usability and computational expense can play a large roll in determining a model's usefulness. The objective of this work is to implement the $\zeta-f$ model and ASBM in the commercial CFD solver FLUENT and validate its performance for canonical turbulent flows including a subsonic turbulent flat- 
plate, S3H4 2D hill, and backward-facing step. Each turbulent flow is evaluated using various turbulence models including Spalart-Allmaras, $k-\varepsilon, k-\omega, k-\omega-\mathrm{SST}, v^{2}-f, \zeta-f$ and two ASBM formulations and compared against experimental results.

\section{Validation Cases}

When implementing any new idea or process, it is important to ensure that implementation behaves as expected. Generally, it is best to segregate properties of the idea and test them separately to help decouple any complexities. For turbulence modeling, it is best to calculate the simplest possible generic flow with one dominating effect. It is also important to use widely accepted cases in order to provide the development community with a reference to compare against. The following cases are well documented in literature and have been used extensively as test cases. Each of the models implemented will be validated on these reference test cases to demonstrate the correct implementation of the turbulence model in FLUENT. The implemented models' accuracy will also be evaluated against experimental data and previously validated models.

\section{Turbulent Flat Plate}

One of the simplest as well as most well documented turbulent flows is the turbulent flat plate. The case has been extensively studied at high Reynolds numbers and many formulas have been proposed for the skin friction as well as the boundary layer profile. Accurate prediction of this case is the basis for accurate prediction on more complex geometries and flows. In the case of aerospace applications, the proper prediction of skin friction is especially important. The skin friction has been predicted experimentally but there is also accepted momentum integral analysis which accurately predicts the skin 
friction coefficient based on Reynolds number. The power law theory will be used as a comparison for skin friction coefficient on the flat plate. It is defined as

$$
C_{f}=\frac{0.027}{\operatorname{Re}_{x}^{1 / 7}}
$$

The self similar boundary layer on a turbulent flat plate has also been extensively studied. The near wall region has been separated into three distinct layers known as the linear sublayer, the buffer region, and the log region. The linear sublayer follows a linear relationship based on non-dimensionalized velocity and wall distance. The log region, of course, follows a logarithmic relation. The buffer region is a smooth connector of the two. The non-dimensionalized variables are defined below.

$$
\begin{gathered}
u^{+}=\frac{u}{v^{*}} \\
y^{+}=\frac{y v^{*}}{v}
\end{gathered}
$$

where $v^{*}$ is the wall-friction velocity defined as

$$
v^{*}=\sqrt{\frac{\tau_{w}}{\rho}}
$$

and $\tau_{w}$ is the wall shear stress. The three layers are then respectively defined by

$$
\begin{array}{cc}
u^{+}=y^{+} & \text {for } y^{+}<5 \\
\text { smooth connection } & \text { for } 5<y^{+}<30 \\
u^{+}=\frac{1}{\kappa} \ln \left(y^{+}\right)+B & \text { for } 30<y^{+}<350
\end{array}
$$

where $\kappa=0.41$ and $B=5.0$. The three layers have been defined in a composite formula which covers the entire wall related region. This formula, called Spaldings formula, is presented below. 


$$
y^{+}=u^{+}+e^{-\kappa B}\left[e^{\kappa u^{+}}-1-\kappa u^{+}-\frac{\left(\kappa u^{+}\right)^{2}}{2}-\frac{\left(\kappa u^{+}\right)^{3}}{6}\right]
$$

The boundary layer profile can also be compared against the experimental data of Wieghardt.

There has also been extensive study of the Reynolds stress profiles throughout the boundary layer. Klebanoff presented data for a flat plate at a Reynolds number of $\operatorname{Re}_{\mathrm{x}}=$ $4.2 \times 10^{6}$. This data will be used for comparison of the Reynolds stresses. Figure 2 below shows the Reynolds stress profiles within the boundary as determined by Klebanoff.

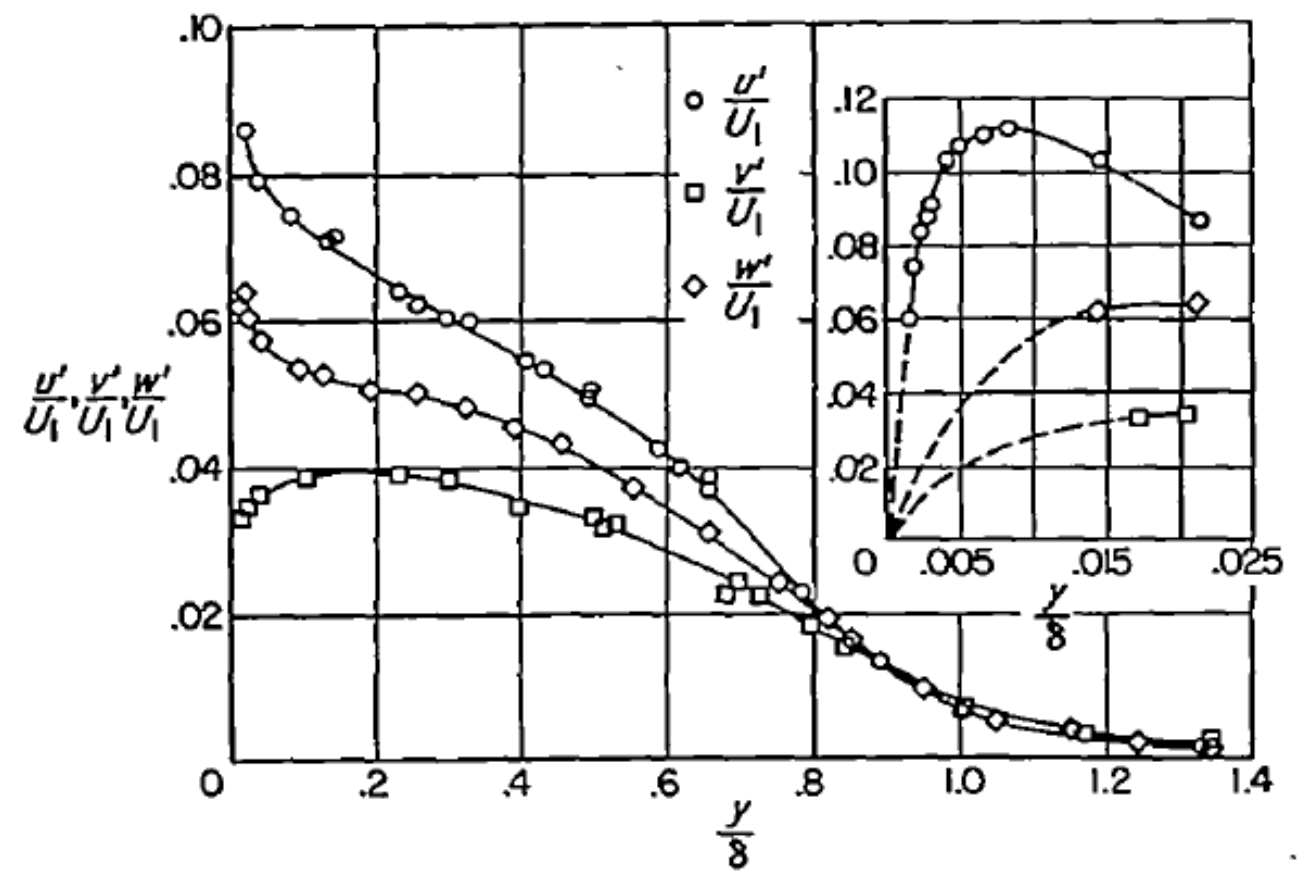

Figure 2. Non-dimensionalized Reynolds stress at $R_{\mathrm{x}}=4.2 \mathrm{e} 6$ by Klebanoff.

\section{Backward Facing Step}

The backward facing step is a canonical test case due to its relative simple geometry but difficult to capture flow physics. Its main purpose is to study the effects of separation and reattachment. While the geometry is simple, the case is actually very computationally expensive due to the required mesh refinement in the step region. The 
point of separation is defined by the geometry at the step. The reattachment position, however, is dependent on the Reynolds number based on step height, $\operatorname{Re}_{h}$. Generally, turbulence models have a difficult time accurately predicting the reattachment length.

The flow can be defined by different regions. Under the step a primary separation region is formed along with a secondary region in the corner. The secondary region is rarely captured in CFD. Between the primary bubble and the mean flow, a shear layer is formed which generates large amounts of turbulent shear stress. After the reattachment point, there is a zone considered the recovery zone which is also hard to predict with turbulence modeling. The recovery zone, however, will not be examined. The geometry and flow can be seen below in Figure 3 .

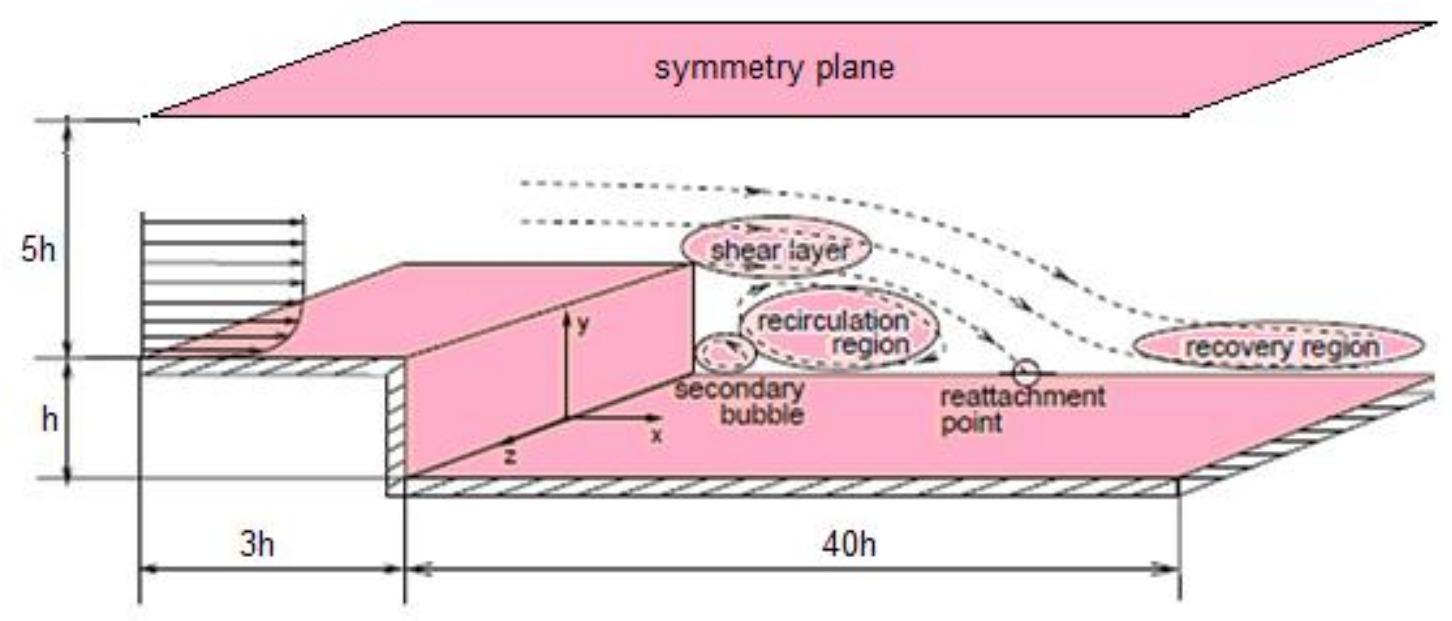

Figure 3. Backward facing step geometry and defining flow characteristics.

The backward facing step calculations were formed with an expansion ration of $H / h=$ 3 with a Reynolds number of $\operatorname{Re}_{h}=5000$ where $\operatorname{Re}_{h}$ is defined as

$$
\operatorname{Re}_{h}=\frac{U_{0} h}{v}
$$


where $h$ is the step height. Experimental data from Jovic and Driver is used to validate the implemented turbulence models. Velocity profiles from the experiment are shown below in Figure 4.

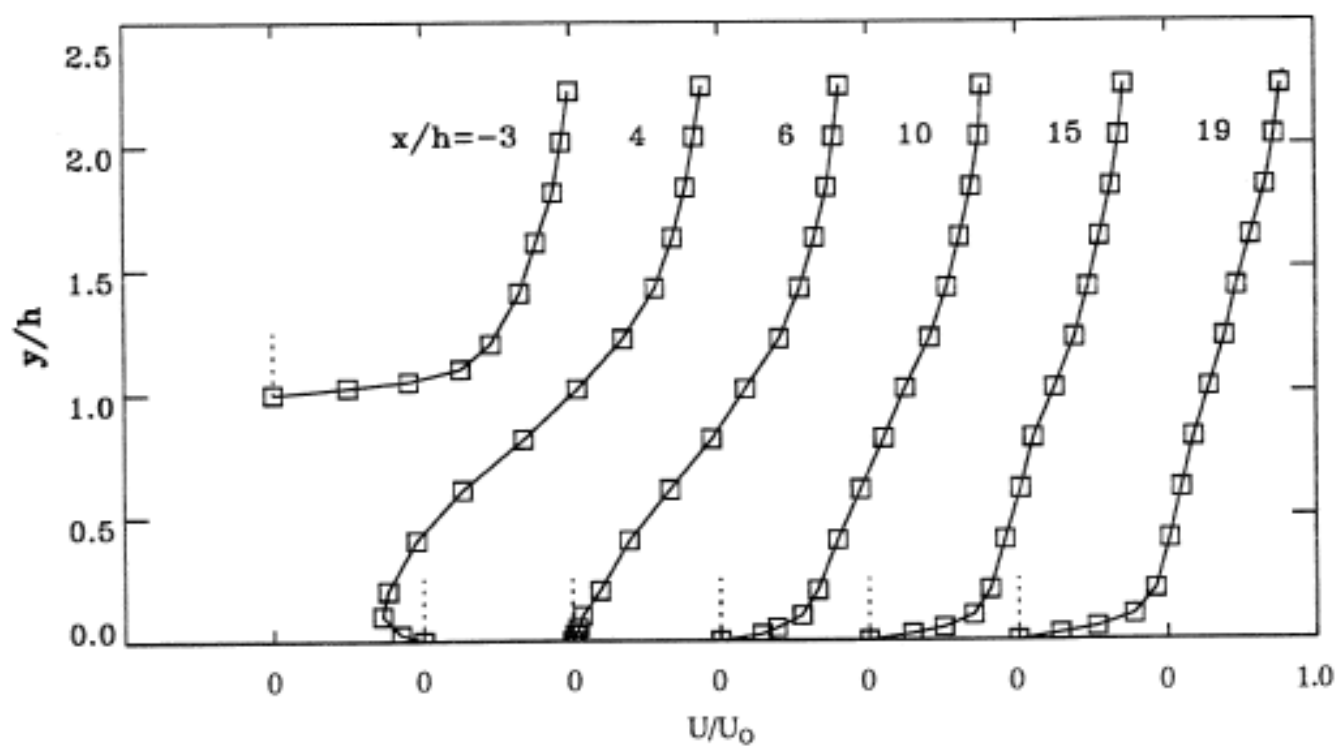

Figure 4. Velocity profiles for a backward facing step by Jovic and Driver.

\section{3. $\mathrm{S} 3 \mathrm{H} 42 \mathrm{D} \mathrm{Hill}$}

The S3H4 2D hill case helps show the effects of streamline curvature and the potential for separation helps display the sensitivity of turbulence models. The hill geometry is defined by Kim et al. where SxHy denotes the maximum slope of 0.x and a height of $y$. The hill geometry is defined as

$$
y=\frac{H}{2}\left\{1+\cos \left[\frac{\pi}{2}\left(\frac{x}{L_{1}}\right)\right]\right\}
$$

where the half length of the hill, $L_{l}$, is defined as

$$
L_{1}=\frac{H}{2 S}
$$

The hill geometry is displayed below in Figure 5. 


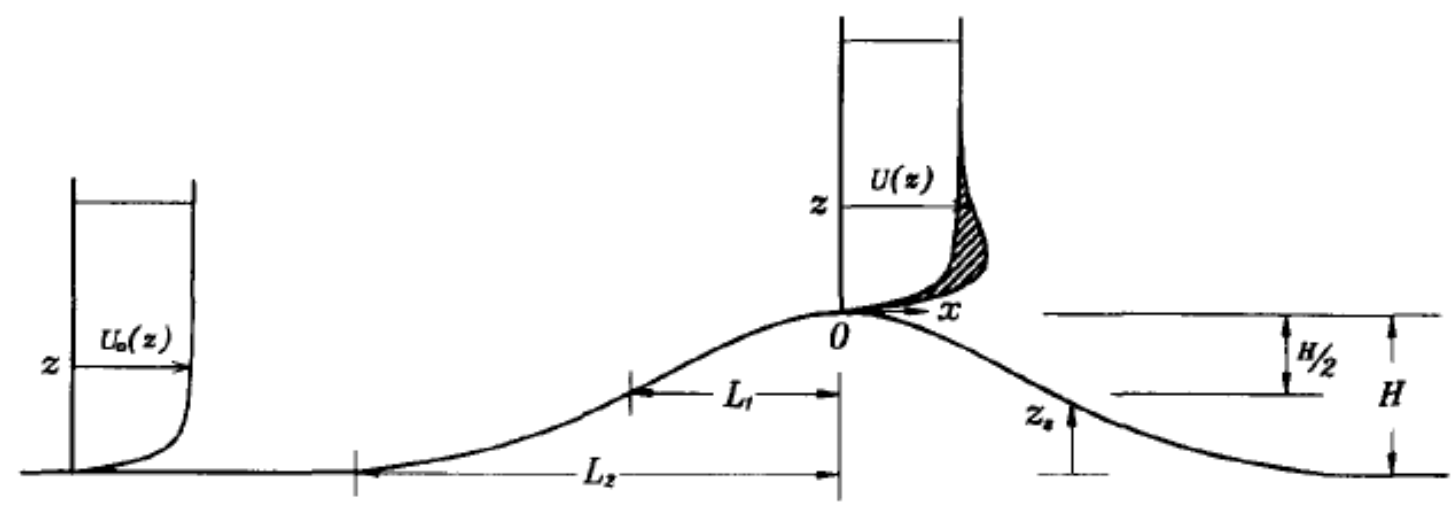

Figure 5. S3H4 2D Hill Geometry by Kim et al.

The $\mathrm{S} 3 \mathrm{H} 4$ hill is the last hill, as the slope and height increase, which does not produce a separation bubble in the region behind the hill. The hill displays high streamline curvature which generally results in equilibrium assumptions of turbulence models not being valid. Therefore, the prediction of flow near the hill can be difficult. Velocity profiles over the hill by Kim et al. are displayed below in Figure 6.

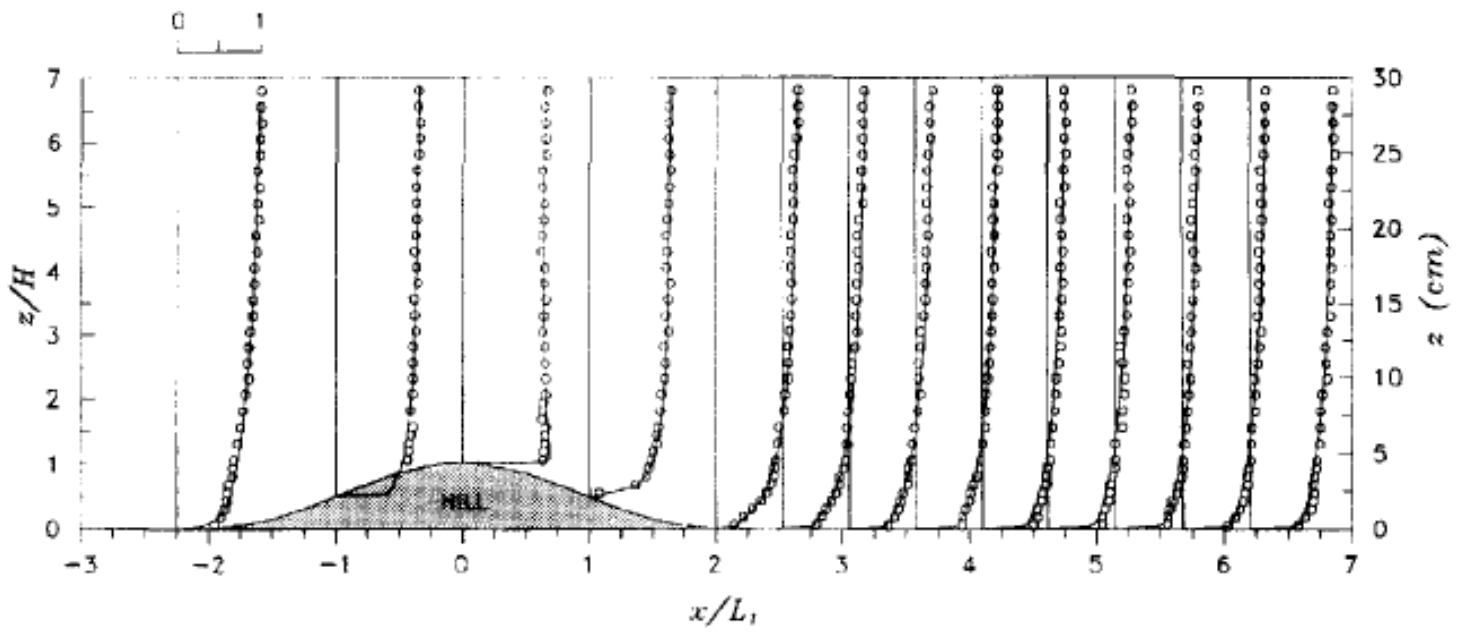

Figure 6. Velocity profiles over the $\mathrm{S3H} 4$ hill by Kim et al.

The experiment was conducted with a boundary layer height Reynolds number of 7 . The boundary layer height Reynolds number is defined as

$$
\operatorname{Re}_{\delta}=\frac{U_{0} \delta}{v}
$$


where $\delta$ is the boundary layer height. To mimic this in CFD, a power law profile was used as the inlet condition for velocity with the proper boundary layer height. 


\section{Turbulence Modeling}

In Computational Fluid Dynamics closure of the laminar Navier-Stokes equations is possible and relatively simple due to the generally large scales defining flow characteristics. This allows for computationally inexpensive meshes to accurately represent the flow. The scales of turbulent flow, however, are relatively small and therefore require extremely computationally expensive meshes to accurately capture flow phenomena. To give an example of the extreme refinement, scales as small as the Kolmogorov scales must be resolved in the mesh. This method is referred to as direct numerical simulation (DNS). DNS requires large amounts of resources, including money and time, to reach a solution as the number of computational operations is proportional to the Reynolds number of the simulation to the third power. Only the simplest geometries at lower Reynolds numbers can generally be solved using DNS. In order to accurately model more complex flows and geometries, only the larger scales are resolved and the small turbulent scales are instead modeled. This is achieved through the ReynoldsAveraged Navier-Stokes equations which introduces extra unknowns. These unknowns are then modeled through the use of turbulence modeling.

\section{A. Governing Equations}

The Reynolds-Averaged Navier-Stokes (RANS) equations are used to obtain flow solutions in the CFD solver FLUENT for turbulent flow. These equations are derived from the fundamental equations of fluid dynamics which include conservation of mass, conservation of momentum, and conservation of energy better known as the full Navier- 
Stokes equations. While the full Navier-Stokes equations present a method to solve the instantaneous flow variables, the RANS equations solve for the time-averaged or Favreaveraged quantities for incompressible and compressible flows respectively. The turbulent fluctuations are used to model turbulence in the flow. To begin the derivation of the RANS equations, each instantaneous flow variable, denoted arbitrarily as $\psi$, is split into its time-averaged and fluctuating values as follows

$$
\psi=\bar{\psi}+\psi^{\prime}
$$

where the bar denotes time-averaging and the prime denotes fluctuating values. The time-average is defined as

$$
\bar{\psi}=\frac{1}{T} \int_{t_{0}}^{t_{0}+T} \psi d t
$$

where $T$ is a large period of time compared to the relevant period of fluctuation.

This expression for the instantaneous flow variable can then be substituted into the full Navier-Stokes equations and simplified based on the basic integral relation. The time-averaged continuity equation can be simplified rather easily and can be written in Cartesian tensor form as:

$$
\frac{\partial \rho}{\partial t}+\frac{\partial}{\partial x_{i}}\left(\rho \bar{u}_{i}\right)=0
$$

The momentum equation, however, is not so simply formulated. It requires the manipulation of both the averaged quantities and fluctuating quantities. The timeaveraged momentum equation can be written in Cartesian tensor form as: 


$$
\begin{aligned}
& \overbrace{\frac{\partial}{\partial t}\left(\rho \bar{u}_{i}\right)}^{\text {unsteady }}+\overbrace{\frac{\partial}{\partial x_{i}}\left(\rho \bar{u}_{i} \bar{u}_{j}\right)}^{\text {convective }}= \\
& \quad-\frac{\partial \bar{p}}{\partial x_{i}}+\frac{\partial}{\partial x_{j}} \underbrace{\left[\mu\left(\frac{\partial \bar{u}_{i}}{\partial x_{j}}+\frac{\partial \bar{u}_{j}}{\partial x_{i}}-\frac{2}{3} \delta_{i j} \frac{\partial \bar{u}_{k}}{\partial x_{k}}\right)\right]}_{\text {laminar }}+\frac{\partial}{\partial x_{j}} \underbrace{\left(-\rho \overline{u_{i}^{\prime} u_{j}^{\prime}}\right)}_{\text {turbulent }}
\end{aligned}
$$

The turbulent inertia, labeled laminar and turbulent, behave as stresses where the laminar portion represents the Newtonian viscous stress and the turbulent portion represents an apparent turbulent stress tensor commonly denoted as the Reynolds stress tensor. Because this new term is never negligible in any turbulent flow, it is imperative to solve for this term. Unfortunately, this is the cause of analytical difficulties for turbulent flows. This tensor introduces nine new terms and six unknowns due to symmetry that need to be accounted for in order to obtain an accurate solution. There is, however, as of yet, no analytic solution for this tensor. It can only be defined by detailed knowledge of turbulent structure. Its components relate to both the fluid physical properties and local flow conditions. Empirical approaches have been attempted with varying levels of success. Most popularly, however, a scalar quantity, the eddy viscosity, is used to partially represent the Reynolds stresses. This technique is known as the Boussinesq approximation.

The Boussinesq approximation is used to compute the Reynolds stress tensor using an eddy viscosity and also the mean strain-rate tensor. This approximation, more accurately an assumption, is based on the fact that the turbulent inertia is represented by a combination of the Newtonian viscous stress and the Reynolds stress tensor. By representing the turbulent contribution to this inertia similarly to that of the laminar contribution, the effect of turbulence on the momentum flux follows the behavior of 
momentum flux on a molecular scale. The Boussinesq approximation for the Reynolds stress tensor is defined as:

$$
-\rho \overline{u_{i}^{\prime} u_{j}^{\prime}}=\mu_{t}\left(\frac{\partial \bar{u}_{i}}{\partial x_{j}}+\frac{\partial \bar{u}_{j}}{\partial x_{i}}\right)-\frac{2}{3}\left(\rho k+\mu_{t} \frac{\partial \bar{u}_{k}}{\partial x_{k}}\right) \delta_{i j}
$$

where the turbulent viscosity, $\mu_{t}$, is defined given a specific turbulence model Some form of this approximation is used in all linear eddy viscosity models (LEVM). Due to its simplicity and overall stability when executed within a solution scheme, the Boussinesq approximation along with various LEVM are used widely. While the Boussinesq approximation is based in physical understanding, it does have limits and these limits are exacerbated in complex flow regimes.

The Boussinesq approximation is fundamentally flawed in complex flow regimes due to the linearization of the relationship between the Reynolds stresses and the strain rate. Defined by a scalar quantity, it is unable to define or even represent complex turbulent structure. In 2D flow, this is exemplified by the dominance of $R_{12}$, the coupling of the fluctuating velocities in the $\mathrm{x}$ and $\mathrm{y}$ direction, in the boundary layer, which is not captured by the approximation. The Boussinesq approximation assumes that the eddy viscosity is isotropic. Expressed mathematically,

$$
\overline{\rho u^{\prime 2}}=\overline{\rho v^{\prime 2}}=\overline{\rho w^{\prime 2}}=\frac{2}{3} \bar{\rho} k
$$

Generally speaking, this component of turbulence structure is rarely accounted for and can actually be quite important to accurately capture flow. Thus more advanced approximations of the Reynolds stresses need to be used to better capture flow behavior. 


\section{B. Common Turbulence Models}

The most commonly used turbulence models in industry as well as in commercial CFD codes generally utilize the Boussinesq approximation. They are computationally inexpensive and very robust. While there are varying levels of accuracy in flow prediction depending on the model used, the models generally produce acceptable results. Some models, however, were developed for specific flow types and are used beyond their intended purpose. This is the major shortcoming of most turbulence models. The most common turbulence models are presented below which will also be used as comparison to the models presented in this thesis.

\section{Spalart-Allmaras Model}

The Spalart-Allmaras model was developed by Spalart and Allmaras in 1992 in order to fill the need for an accurate, extremely computationally inexpensive, and robust model. The model is a one equation model created based heavily on experimental results and motivated by flaws with existing turbulence models. First, the model was meant to replace the zero equation models such as the Baldwin-Lomax model by increasing robustness with little increase in computational expense. Second, it was designed to predict nearly as accurately as common two equation models, such as the $k-\varepsilon$ model, while avoiding the use of strong source terms which tend to delay convergence. Lastly, many two equation models require boundary layer meshes to be resolved to within the viscous sublayer which can be more computationally expensive. The Spalart-Allmaras model was developed to allow for less resolved meshes and thus fewer resources. It was also created specifically for aerospace applications including those subject to the adverse pressure gradients. 
The Spalart-Allmaras model utilizes one additional transport equation in order to model turbulent effects. The transport variable represents the undamped turbulent eddy viscosity and is denoted by $\tilde{v}$ where the tilde is not a Favre averaged quantity. The transport equation is defined as follows

$$
\begin{aligned}
& \frac{\partial(\rho \tilde{v})}{\partial t}+\frac{\partial\left(\rho \tilde{v} u_{i}\right)}{\partial x_{i}}= \\
& P_{\tilde{v}}-Y_{\tilde{v}} \\
&+\frac{1}{\sigma_{\tilde{v}}}\left[\frac{\partial}{\partial x_{j}}\left\{(\mu+\rho \tilde{v}) \frac{\partial}{\partial x_{j}}\right\}+C_{b 2} \rho\left(\frac{\partial \tilde{v}}{\partial x_{j}} \frac{\partial \tilde{v}}{\partial x_{j}}\right)\right]
\end{aligned}
$$

where $P_{\tilde{v}}$ is the production of turbulent viscosity, $Y_{\tilde{v}}$ is the destruction of turbulent viscosity, $\mu$ is the dynamic viscosity, and $\sigma_{\tilde{v}}$ and $C_{b 2}$ are model constants to be defined.

The turbulent viscosity, $\mu_{t}$, used in the Boussinesq approximation is computed using a viscous damping function as

$$
\mu_{t}=\rho \tilde{v} f_{v 1}
$$

where $f_{v l}$ is a viscous damping function defined as

$$
f_{v 1}=\frac{\left(\frac{\tilde{v}}{v}\right)^{3}}{\left(\frac{\tilde{v}}{v}\right)^{3}+C_{v 1}^{3}}
$$

with $v$ as the kinematic viscosity and $C_{v l}$ representing a calibrated constant.

The production of turbulent viscosity is defined as

$$
P_{\tilde{v}}=C_{b 1} \rho\left[\Omega+\frac{\tilde{v}}{\kappa^{2} d^{2}}\left(1-\frac{\frac{\tilde{v}}{v}}{1+\frac{\tilde{v}}{v} f_{v 1}}\right)\right] \tilde{v}
$$


where $d$ is the distance from the wall, $\Omega$ is a scalar measure of the deformation tensor, $C_{b 1}$ is a calibrated constant, and $\kappa$ is the von Karman constant commonly used to define the $\log$ region of boundary layer flow which is approximately equal to 0.41 . The original Spalart-Allmaras model defined $\Omega$ as the magnitude of mean rotation rate tensor as described below

$$
\Omega=\sqrt{2 \Omega_{i j} \Omega_{i j}}
$$

where $\Omega_{i j}$ is the mean rotation rate tensor,

$$
\Omega_{i j}=\frac{1}{2}\left(\frac{\partial u_{i}}{\partial x_{j}}-\frac{\partial u_{j}}{\partial x_{i}}\right)
$$

Spalart and Allmaras justified the use of the mean rotation rate tensor based on the claim that for wall bounded flows, turbulence is found only where vorticity is generated near walls. Other definitions of for the scalar measure of the deformation tensor have been proposed, however.

The destruction of turbulence term is modeled as

$$
Y_{\tilde{v}}=C_{w 1} \rho f_{w}\left(\frac{\tilde{v}}{d}\right)^{2}
$$

where $f_{w}$ is another viscous damping function defined as

$$
\begin{gathered}
f_{w}=g\left[\frac{1+C_{w 3}^{6}}{g^{6}+C_{w 3}^{6}}\right]^{1 / 6} \\
g=r+C_{w 2}\left(r^{6}-r\right) \\
r=\frac{\tilde{v}}{\left(\Omega+\frac{\tilde{v}}{\kappa^{2} d^{2}} f_{v 2}\right) \kappa^{2} d^{2}}
\end{gathered}
$$

and $C_{w 1}, C_{w 2}$, and $C_{w 3}$ are calibrated constants which can vary. 
As stated before, the model was created for aerospace applications with adverse pressure gradients and thus predicts very well for simple attached flows without separation regions. In more complex flows, however, especially those with separation, the model predicts poorly and can even become less robust than the common two equation models. This is also true in flows with high streamline curvature such as $2 \mathrm{D}$ hills and more advanced airfoil geometries. Wilcox also shows that the Spalart-Allmaras model predicts free shear regions poorly. He concluded that it should not be used for jetlike free shear layers although this will not be studied here. Also, the model is coupled to mean flow through the Boussinesq approximation which is unable to properly capture Reynolds stress anisotropy which is increasingly important in complex flows.

\section{2. $k-\varepsilon$ Model}

The $k-\varepsilon$ model is a two equation model with initial development stemming back to the origins of turbulence models. The standard $k-\varepsilon$ model was developed by Launder and Sharma and includes calibrated closure coefficients. References to the "standard" model refer to the specific set of constants determined by aforementioned authors. The model is a canonical model that is often criticized but still widely used. It utilizes transport equations for the turbulent kinetic energy, $k$, and the turbulence dissipation rate, $\varepsilon$. The turbulent kinetic energy equation is derived directly from contracting the differential equation of the Reynolds-stress tensor and is purely mathematical. The $\varepsilon$ transport equation, however, is derived through physical reasoning and matching the form of the turbulent kinetic energy due to the lack of experimental data related to the exact transport equation. Wilcox criticizes this method stating, "the relation between the modeled equation for $\varepsilon$ and the exact equation is so tenuous as not to need serious consideration.” 
Despite this criticism, the model generally predicts well. The transport equations are displayed below.

$$
\begin{gathered}
\mu_{t}=\rho C_{\mu} \frac{k^{2}}{\varepsilon} \\
\frac{\partial(\rho k)}{\partial t}+\frac{\partial\left(\rho k u_{i}\right)}{\partial x_{i}}=P_{k}-\rho \varepsilon-Y_{M}+\frac{\partial}{\partial x_{j}}\left[\left(\mu+\frac{\mu_{t}}{\sigma_{k}}\right) \frac{\partial k}{\partial x_{j}}\right] \\
\frac{\partial(\rho \varepsilon)}{\partial t}+\frac{\partial\left(\rho \varepsilon u_{i}\right)}{\partial x_{i}}=C_{1 \varepsilon} \frac{\varepsilon}{k}\left(P_{k}+C_{3 \varepsilon} G_{b}\right)-C_{2 \varepsilon} \rho \frac{\varepsilon^{2}}{k} \\
+\frac{\partial}{\partial x_{j}}\left[\left(\mu+\frac{\mu_{t}}{\sigma_{\varepsilon}}\right) \frac{\partial \varepsilon}{\partial x_{j}}\right]
\end{gathered}
$$

In the transport equations above, $\mu_{t}$ is the turbulent viscosity and $C_{1 \varepsilon}, C_{2 \varepsilon}$, and $C_{3 \varepsilon}$ are calibrated constants. Within the diffusive terms on the right side of the transport equations, $\sigma_{k}$ and $\sigma_{\varepsilon}$ are the turbulent Prandtl numbers for $k$ and $\varepsilon$ respectively. $P_{k}$ represents the production of turbulent kinetic energy and is modeled as

$$
P_{k}=\mu_{t} S^{2}
$$

where

$$
S=\sqrt{2 S_{i j} S_{i j}}
$$

and

$$
S_{i j}=\frac{1}{2}\left(\frac{\partial u_{i}}{\partial x_{j}}+\frac{\partial u_{j}}{\partial x_{i}}\right)
$$

$Y_{M}$ accounts for compressibility and is defined as

$$
Y_{M}=2 \rho \varepsilon M_{t}^{2}
$$

where 


$$
M_{t}=\sqrt{\frac{k}{a^{2}}}
$$

Lastly, the effects of buoyancy are represented by $G_{b}$. For all simulations in this work buoyancy effects were neglected, however. The constants were previously calibrated such that the model matches experimental data for both air and water.

While the model was calibrated to match experimental data, its performance on flows extending beyond that which it was calibrated for can be lacking. It is well known that the ad hoc formulation of the turbulence dissipation equation leads to poor prediction for wall bounded adverse pressure gradient flows. Again the Boussinesq approximation limits its applicability to complex flows where Reynolds stress anisotropy becomes more dominate. To extend the model to more flows, damping functions can be applied which of course improve prediction for certain problems but are not a general solution to the modeling problem. Bell concluded in a series of test cases that this model is not ideal for flows with separation and low Reynolds number effects. Additionally, the model cannot be integrated to the wall and requires the use of empirical wall functions to model boundary layer flows.

Wall functions can also have a large effect on the flow solutions and of course have a large effect on skin friction coefficient prediction, a very important solution result for aerospace applications. For this study, enhanced wall treatment, available in FLUENT, was used which is described below.

The enhanced wall treatment is a two-layer approach which is used to calculate both turbulent dissipation and turbulent viscosity in near-wall cells. The two-layer approach subdivides the entire domain into a wall affected region and a fully turbulent region. In this method, these regions are determined by the turbulent Reynolds number defined as 


$$
\operatorname{Re}_{y} \equiv \frac{\rho y \sqrt{k}}{\mu}
$$

where $y$ is the wall normal distance.

In the fully turbulent region where $\mathrm{Re}_{y}$ is greater than 200 , the standard $k-\varepsilon$ equations described above are implemented. In the wall affected region, however, where $\operatorname{Re}_{y}$ is less than 200, a one equation model is employed. The turbulent kinetic energy transport equation is retained but an algebraic model is used to predict $\varepsilon$. The turbulent viscosity in this region is defined as

$$
\mu_{t, 2 \mathrm{layer}}=\rho C_{\mu} \ell_{\mu} \sqrt{k}
$$

where the length scale is computed as

$$
\ell_{\mu}=y C_{\ell}^{*}\left(1-e^{-\mathrm{Re}_{y} / A_{\mu}}\right)
$$

The turbulent viscosity needs to blended, however, such that there are no discontinuities between the two regions. A blending function is used such that it is unity far from walls and zero near wall. The function is displayed below.

$$
\lambda_{\varepsilon}=\frac{1}{2}\left[1+\tanh \left(\frac{\operatorname{Re}_{y}-200}{A}\right)\right]
$$

where the constant $A$ is defined as

$$
A=\frac{\left|\Delta \operatorname{Re}_{y}\right|}{\tanh (0.98)}
$$

and $\Delta \operatorname{Re}_{y}$ has a value between 10 and 40.

Finally, the turbulent dissipation is computed from

$$
\varepsilon=\frac{k^{3 / 2}}{\ell_{\varepsilon}}
$$


and the length scale is

$$
\ell_{\varepsilon}=y C_{\ell}^{*}\left(1-e^{-\mathrm{Re}_{y} / A_{\varepsilon}}\right)
$$

The constants used in this wall function are shown below.

$$
C_{\ell}^{*}=\kappa C_{\mu}^{-3 / 4}, \quad A_{\mu}=70, \quad A_{\varepsilon}=2 C_{\ell}^{*}
$$

\section{3. $k-\omega$ Model}

The $k-\omega$ model was developed by Wilcox and is very popular. It was created in response to shortcomings of the previously described $k$ - $\varepsilon$ model. The model includes transport equations for the turbulent kinetic energy and the specific dissipation rate. The $k-\omega$ model also does not require wall functions but this is due to its empirical rather than mathematical basis. It has been modified largely on an ad-hoc basis in order to improve accuracy on a larger range of flows. This is achieved through the utilization of damping functions which are entirely non-physical. While this is advantageous for near wall regions, the model still has shortcomings which will be discussed later. Due to its nonphysical formulation, many different implementations exist.

The model is defined by scalar transport equations for the turbulence kinetic energy and the specific dissipation rate shown below.

$$
\begin{aligned}
& \frac{\partial(\rho k)}{\partial t}+\frac{\partial\left(\rho k u_{i}\right)}{\partial x_{i}}=P_{k}-Y_{k}+\frac{\partial}{\partial x_{j}}\left[\left(\mu+\frac{\mu_{t}}{\sigma_{k}}\right) \frac{\partial k}{\partial x_{j}}\right] \\
& \frac{\partial(\rho \omega)}{\partial t}+\frac{\partial\left(\rho \omega u_{i}\right)}{\partial x_{i}}=P_{\omega}-Y_{\omega}+\frac{\partial}{\partial x_{j}}\left[\left(\mu+\frac{\mu_{t}}{\sigma_{\omega}}\right) \frac{\partial \omega}{\partial x_{j}}\right]
\end{aligned}
$$

The $P_{k}$ term represents the production of turbulence and is identical to the previous definitions. The production term in the specific dissipation equation is modified slightly with a damping function, however. 


$$
\begin{gathered}
P_{k}=\mu_{t} S^{2} \\
P_{\omega}=\alpha \frac{\omega}{k} P_{k}
\end{gathered}
$$

where $\alpha$ is a damping function defined as

$$
\alpha=\frac{\alpha_{\infty}}{\alpha^{*}}\left(\frac{\alpha_{0}+\frac{\mathrm{Re}_{t}}{R_{\omega}}}{1+\frac{\mathrm{Re}_{t}}{R_{\omega}}}\right)
$$

and $R_{\omega}$ and $\alpha_{0}$ are calibrated constants and $\operatorname{Re}_{t}$ is a turbulent Reynolds number.

$$
\operatorname{Re}_{t}=\frac{\rho k}{\mu \omega}
$$

Another damping function, $\alpha^{*}$, partially defines the turbulent viscosity as well as the previously mentioned damping function.

$$
\alpha^{*}=\alpha_{\infty}^{*}\left(\frac{\alpha_{0}^{*}+\frac{\mathrm{Re}_{t}}{R_{k}}}{1+\frac{\mathrm{Re}_{t}}{R_{k}}}\right)
$$

The turbulent viscosity is then defined as

$$
\mu_{t}=\alpha^{*} \frac{\rho k}{\omega}
$$

The turbulence dissipation is modeled as

$$
Y_{k}=\rho \beta^{*} f_{\beta^{*}} k \omega
$$

where

$$
f_{\beta^{*}}=\left\{\begin{array}{cc}
1 & \chi_{k} \leq 0 \\
\frac{1+680 \chi_{k}^{2}}{1+400 \chi_{k}^{2}} & \chi_{k}>0
\end{array}\right.
$$




$$
\chi_{k}=\frac{1}{\omega^{3}} \frac{\partial k}{\partial x_{j}} \frac{\partial \omega}{\partial x_{j}}
$$

and $\beta^{*}$ is a function of calibrated constants, the turbulent Reynolds number and a compressibility correction.

$$
\begin{gathered}
\beta^{*}=\beta_{i}^{*}\left[1+\zeta^{*} F\left(M_{t}\right)\right] \\
\beta_{i}^{*}=\beta_{\infty}^{*}\left(\frac{4 / 15+\left(\operatorname{Re}_{t} / R_{\beta}\right)^{4}}{1+\left(\operatorname{Re}_{t} / R_{\beta}\right)^{4}}\right) \\
\zeta^{*}=1.5 \quad R_{\beta}=8 \quad \beta_{\infty}^{*}=0.09
\end{gathered}
$$

The dissipation of specific dissipation rate is modeled as

$$
Y_{\omega}=\rho \beta f_{\beta} \omega^{2}
$$

where

$$
\begin{gathered}
f_{\beta}=\frac{1+70 \chi_{\omega}}{1+80 \chi_{\omega}} \\
\chi_{\omega}=\left|\frac{\Omega_{i j} \Omega_{j k} S_{k i}}{\left(\beta_{\infty}^{*} \omega\right)^{3}}\right|
\end{gathered}
$$

and $\beta$ is a function of calibrated constants similar to $\beta^{*}$.

$$
\beta=\beta_{i}\left[1-\frac{\beta_{i}^{*}}{\beta_{i}} \zeta^{*} F\left(M_{t}\right)\right]
$$

Both dissipation terms account for compressibility in high Mach-number flows through the use of a compressibility function. This function is a simple adjustment given the turbulent Mach number.

$$
F\left(M_{t}\right)=\left\{\begin{array}{cc}
0 & M_{t} \leq M_{t 0} \\
M_{t}^{2}-M_{t 0}^{2} & M_{t}>M_{t 0}
\end{array}\right.
$$


where the turbulent Mach number is defined as

$$
\begin{aligned}
& M_{t} \equiv \sqrt{\frac{2 k}{a^{2}}} \\
& a=\sqrt{\gamma R T} \\
& M_{t 0}=0.25
\end{aligned}
$$

The calibrated constants as used throughout the model are displayed below

$$
\begin{aligned}
& \alpha_{\infty}^{*}=1 \quad \alpha_{\infty}=0.52 \quad \alpha_{0}=\frac{1}{9} \quad \beta_{\infty}^{*}=0.09 \quad \beta_{i}=0.072 \\
& R_{k}=6 \quad R_{\omega}=2.95 \quad \sigma_{k}=2.0 \quad \sigma_{\omega}=2.0
\end{aligned}
$$

As previously mentioned this model's major shortcomings stem from its ad-hoc formulation. The use of damping functions has lost popularity due to their non-physical formulation. While the model has been adjusted to accurately predict certain flows, it is less able to work as a general model due to its specific formulation. In studies by Bell, it was concluded that $k$ - $\omega$ does generally predict better than the previous two models but is still limited due to its experimental formulation. As with the previously mentioned turbulence models, this model also utilizes the Boussinesq approximation which fails to model Reynolds stress anisotropy.

\section{4. $k-\omega$ SST Model}

The $k-\omega$ SST model was developed by Menter in order to improve the free shear flow performance of the $k$ - $\omega$ model. In essence, the model works just as the $k-\varepsilon$ model far from the wall and like the $k-\omega$ model near the wall. This incorporates the best of both models to make a superior model. While the model is a blending, it takes the form of a $k$ $\omega$ model with refinement. The standard $k-\omega$ model and a transformed $k-\varepsilon$ model are multiplied by a blending function and added together. The blending function activates 
the $k-\omega$ model near wall and activates the transformed $k-\varepsilon$ model far from the wall. The SST model also utilizes a damped cross-diffusion derivative term. The transport equations are described below.

$$
\begin{gathered}
\frac{\partial(\rho k)}{\partial t}+\frac{\partial\left(\rho k u_{i}\right)}{\partial x_{i}}=\tilde{P}_{k}-Y_{k}+\frac{\partial}{\partial x_{j}}\left[\left(\mu+\frac{\mu_{t}}{\sigma_{k}}\right) \frac{\partial k}{\partial x_{j}}\right] \\
\frac{\partial(\rho \omega)}{\partial t}+\frac{\partial\left(\rho \omega u_{i}\right)}{\partial x_{i}}=P_{\omega}-Y_{\omega}+D_{\omega}+\frac{\partial}{\partial x_{j}}\left[\left(\mu+\frac{\mu_{t}}{\sigma_{\omega}}\right) \frac{\partial \omega}{\partial x_{j}}\right]
\end{gathered}
$$

In the above transport equation the production of turbulent kinetic energy is defined as

$$
\tilde{P}_{k}=\min \left(P_{k}, 10 \rho \beta^{*} k \omega\right)
$$

where

$$
P_{k}=\mu_{t} S^{2}
$$

and the turbulent viscosity is computed from

$$
\mu_{t}=\frac{\rho k}{\omega} \frac{1}{\max \left(\frac{1}{\alpha^{*}}, \frac{S F_{2}}{\alpha_{1} \omega}\right)}
$$

$S$ is the strain rate magnitude which was defined previously. The turbulent Prandtl numbers are defined as

$$
\begin{gathered}
\sigma_{k}=\frac{1}{F_{1} / \sigma_{k, 1}+\left(1-F_{1}\right) / \sigma_{k, 2}} \\
\sigma_{\omega}=\frac{1}{F_{1} / \sigma_{\omega, 1}+\left(1-F_{1}\right) / \sigma_{\omega, 2}}
\end{gathered}
$$

$\alpha^{*}$ is defined the same as in the $k$ - $\omega$ model. The blending functions are given by

$$
F_{1}=\tanh \left(\Phi_{1}^{4}\right)
$$




$$
\begin{gathered}
\Phi_{1}=\min \left[\max \left(\frac{\sqrt{k}}{0.09 \omega y}, \frac{500 \mu}{\rho y^{2} \omega}\right), \frac{4 \rho k}{\sigma_{\omega, 2} D_{\omega}^{+} y^{2}}\right] \\
D_{\omega}^{+}=\max \left[2 \rho \frac{1}{\sigma_{\omega, 2}} \frac{1}{\omega} \frac{\partial k}{\partial x_{j}} \frac{\partial \omega}{\partial x_{j}}, 10^{-10}\right] \\
F_{2}=\tanh \left(\Phi_{2}^{2}\right) \\
\Phi_{2}=\max \left[2 \frac{\sqrt{k}}{0.09 \omega y}, \frac{500 \mu}{\rho y^{2} \omega}\right]
\end{gathered}
$$

The production of specific dissipation is defined as

$$
P_{\omega}=\frac{\alpha}{v_{t}} P_{k}
$$

The dissipation terms for both the $k$ and the $\omega$ equations are defined below.

$$
\begin{gathered}
Y_{k}=\rho \beta^{*} k \omega \\
Y_{\omega}=\rho \beta_{i} \omega^{2}
\end{gathered}
$$

where

$$
\beta_{i}=F_{1} \beta_{i, 1}+\left(1-F_{1}\right) \beta_{i, 2}
$$

is defined by the blending function $F_{1} . \alpha_{\infty}$ is redefined as

$$
\alpha_{\infty}=F_{1} \alpha_{\infty, 1}+\left(1-F_{1}\right) \alpha_{\infty, 2}
$$

and

$$
\begin{gathered}
\alpha_{\infty, 1}=\frac{\beta_{i, 1}}{\beta_{\infty}^{*}}-\frac{\kappa^{2}}{\sigma_{\omega, 1} \sqrt{\beta_{\infty}^{*}}} \\
\alpha_{\infty, 2}=\frac{\beta_{i, 2}}{\beta_{\infty}^{*}}-\frac{\kappa^{2}}{\sigma_{\omega, 2} \sqrt{\beta_{\infty}^{*}}}
\end{gathered}
$$

Lastly, the cross diffusion term is written as 


$$
D_{\omega}=2\left(1-F_{1}\right) \rho \sigma_{\omega, 2} \frac{1}{\omega} \frac{\partial k}{\partial x_{j}} \frac{\partial \omega}{\partial x_{j}}
$$

The model constants are displayed below.

$$
\begin{gathered}
\sigma_{k, 1}=1.176, \quad \sigma_{\omega, 1}=2.0, \quad \sigma_{k, 2}=1.0, \quad \sigma_{\omega, 2}=1.168, \\
\alpha_{1}=0.31, \quad \beta_{i, 1}=0.075, \quad \beta_{i, 2}=0.0828
\end{gathered}
$$

Other model constants that aren't defined here are identical to those previously defined in the $k-\omega$ model.

The $k-\omega$-SST model is generally considered the best performing of the two-equation models. It is more accurate in complex flows including separation and comparable to other models in simple flows. It does, however, have its difficulties capturing wake flow accurately. Additionally, it is not coordinate invariant meaning that it is dependent on the distance from the wall. While this is not necessarily problematic computationally, it is less desirable ideologically as this makes the model less general. It also introduces ambiguities in definition of the wall distance.

\section{5. $v^{2}-f$ Model}

The $v^{2}-f$ model was initially developed by Durbin for flows in which near-wall turbulence is of great significance. This includes flows with separation, recirculation and heat transfer. This is a more advanced model which solves four additional transport equations. The transport equations are for the turbulent kinetic energy, the turbulent dissipation rate, the velocity scale, and the elliptic relaxation factor. The model is an extension of the $k-\varepsilon$ model which utilizes a new velocity scale and also the elliptic relaxation factor to capture global effects throughout the domain. The model can be integrated to the wall eliminating the need for wall functions or damping functions. The 
velocity scale allows the model to scale the damping effects of the turbulent transport near wall which the turbulent kinetic energy transport equation is incapable of doing.

The model stems from observation that the correct time scale is the ratio of $k$ and $\varepsilon$ but the turbulent kinetic energy is the incorrect velocity scale. This led to the introduction of the $v^{2}$ transport equation. In addition, it was observed that inviscid blocking of the velocity scale has an effect even far from the wall. Thus there are nonlocal effects that should be included in the determining local flow properties. An elliptic relaxation equation was employed to capture these non-local effects of wall blockage. Generally, the $v^{2}-f$ model has proven to be a very accurate model although it is more computationally expensive than the previously mentioned models.

The $v^{2}-f$ model utilizes similar transport equations for the turbulent kinetic energy and the turbulent dissipation as the $k-\varepsilon$ model. The four transport equations for the model are presented below.

$$
\begin{gathered}
\frac{\partial(\rho k)}{\partial t}+\frac{\partial\left(\rho k u_{i}\right)}{\partial x_{i}}=P_{k}-\rho \varepsilon+\frac{\partial}{\partial x_{j}}\left[\left(\mu+\frac{\mu_{t}}{\sigma_{k}}\right) \frac{\partial k}{\partial x_{j}}\right] \\
\frac{\partial(\rho \varepsilon)}{\partial t}+\frac{\partial\left(\rho \varepsilon u_{i}\right)}{\partial x_{i}}=\frac{C_{1 \varepsilon} P_{k}-C_{2 \varepsilon} \rho \varepsilon}{T}+\frac{\partial}{\partial x_{j}}\left[\left(\mu+\frac{\mu_{t}}{\sigma_{\varepsilon}}\right) \frac{\partial \varepsilon}{\partial x_{j}}\right] \\
\frac{\partial\left(\rho \overline{v^{2}}\right)}{\partial t}+\frac{\partial\left(\rho \bar{v}^{2} u_{i}\right)}{\partial x_{i}}=\rho k f-6 \rho \overline{v^{2}} \frac{\varepsilon}{k}+\frac{\partial}{\partial x_{j}}\left[\left(\mu+\frac{\mu_{t}}{\sigma_{k}}\right) \frac{\partial \overline{v^{2}}}{\partial x_{j}}\right] \\
L^{2} \frac{\partial^{2} f}{\partial x_{k} \partial x_{k}}-f=\frac{1}{T}\left(C_{1}-1\right)\left(\frac{\overline{v^{2}}}{k}-\frac{2}{3}\right)-C_{2} \frac{P_{k}}{\rho k}
\end{gathered}
$$

where $P_{k}$ is again the production of turbulence kinetic energy due to the mean flow velocity gradients. 


$$
P_{k}=\mu_{t} S^{2}
$$

$T$ is a measure of the turbulence time scale. Away from solid walls the estimate for the time scale, $k / \varepsilon$, is reasonable. Near wall, however, this estimate drops below the Kolmogorov scales. The turbulent time scale cannot become less than the Kolmogorov scale and thus $T$ is defined as follows,

$$
T=\max \left[\frac{k}{\varepsilon}, C_{T} \sqrt{\frac{\mu}{\rho \varepsilon}}\right]
$$

where the first term is a measure of the turbulent time scale and the second term is a measure of the Kolmogorov scale. The turbulence length scale is defined similarly again requiring that the scale is larger than that of the Kolmogorov scale. It is defined as follows.

$$
L=C_{L} \max \left[\frac{k^{3 / 2}}{\varepsilon}, C_{\eta}\left(\frac{\mu^{3}}{\rho^{3} \varepsilon}\right)^{1 / 4}\right]
$$

The turbulence viscosity is defined by the velocity scale $v^{2}$ and the turbulent time scale $T$.

$$
\mu_{t}=\rho C_{\mu} \overline{v^{2}} T
$$

Finally, the model constants have been calibrated based on previous work in order to best fit turbulent flat plate boundary layer results. These constants differ slightly than other published data but have been shown to produce accurate predictions. The result of this calibration is shown below.

$$
\begin{gathered}
C_{\mu}=0.2 \quad \sigma_{k}=1 \quad \sigma_{\varepsilon}=1.3 \quad C_{1}=1.6 \quad C_{2}=0.3 \\
C_{T}=6 \quad C_{L}=0.23 \quad C_{\eta}=60
\end{gathered}
$$


The $v^{2}-f$ model performs significantly better than the previously mentioned models at a relatively low price. It does, however, still have its flaws. This is mainly due to mesh sensitivity near wall and also robustness. Robustness is especially important for generalized flow solvers. Additionally, the segregated solver used in FLUENT make this model more susceptible to divergence. The Boussinesq approximation is also used to couple this model to mean flow. 


\section{III. ל-f Turbulence Model}

The $\zeta-f$ model was developed in order to overcome the shortcomings of the $v^{2}-f$ model developed by Durbin. While the Durbin model significantly improved the flow prediction as compared to industry standards such as the standard $k$ - $\varepsilon$ model, widespread use is not common due to its restrictive abilities in complex flows. This includes a lack of robustness in complex geometries, boundary conditions for $f$, the elliptic relaxation equation, and also, mesh sensitivity related to the $f$ boundary condition. Previously, attempts have been made to improve these issues especially related to stability and robustness.

Improvements to the $v^{2}-f$ model have come from multiple publications and addressed some of the previously mentioned problems. Durbin initially modified the definitions of the turbulent time and length scales to better represent appropriate flow behavior in differing conditions. Due to their large influence, this increased predictive capabilities of the model. The $f$ elliptic relaxation equation was also reformulated by Lien et al. in order to remove grid dependence and improve stability. It does, however, result in slightly worse model predictive behavior. Another notable modification was made by Davidson which forced $v^{2}$ to be less than $2 / 3 k$, the isotropic flow condition. This modification has been criticized due to its lack of elegance and the fact that the limitation is not always correct. It does, however, increase stability. While these modifications have slowly improved the model, the $\zeta-f$ model aims to reformulate the transport equations to increase robustness. 
Popovac and Hanjalic have developed the $\zeta-f$ model as a modification to the Durbin model which solves for the transport equation of the velocity scale ratio $\zeta=v^{2} / k$ as opposed to the velocity scale $v^{2}$. Previous work has shown that this transformation in the transport equation results in better numerical stability. This is partially due to reduced gradients throughout the flow field. Another modification, made to the $f$ equation, also seems to result in better prediction.

\section{A. ל Equation}

The $v^{2}$ equation was modified by the introduction of a new variable, the normalized wall-normal velocity scale $\zeta=v^{2} / k$. Popovac describes this turbulence variable as the ratio of the isotropic scalar time scale, $k / \varepsilon$, and the anisotropic lateral time scale, $v^{2} / \varepsilon$. The $\zeta$ transport equation is derived from the $v^{2}$ and $k$ equations as follows.

$$
\begin{aligned}
\frac{D \zeta}{D t}=\frac{D\left(\overline{v^{2}} / k\right)}{D t}= & \frac{1}{k^{2}}\left(k \frac{D \overline{v^{2}}}{D t}-\overline{v^{2}} \frac{D k}{D t}\right)=\frac{1}{k} \frac{D \overline{v^{2}}}{D t}-\frac{\overline{v^{2}}}{k^{2}} \frac{D k}{D t} \\
= & \frac{1}{k}\left\{k f-\varepsilon \frac{\overline{v^{2}}}{k}+\frac{\partial}{\partial x_{k}}\left[\left(v+v_{t}\right) \frac{\partial \overline{v^{2}}}{\partial x_{k}}\right]\right\}- \\
& \left.\frac{\overline{v^{2}}}{k^{2}}\left\{\frac{P_{k}}{\rho}-\varepsilon+\frac{\partial}{\partial x_{k}}\left[\left(v+v_{t}\right) \frac{\partial k}{\partial x_{k}}\right]\right\}\right\} \\
= & f-\frac{\partial\left(\overline{v^{2}} / k\right)}{k^{2}} \frac{P_{k}}{\rho}+\frac{\partial}{\partial x_{k}}\left[\left(v+v_{t}\right) \frac{\partial x_{k}}{\partial\left(v+v_{t}\right) \frac{\partial\left(\overline{v^{2}} / k\right)}{\partial x_{k}} \frac{\partial k}{\partial x_{k}}}\right.
\end{aligned}
$$

where $P_{k}$ is the production of turbulent kinetic energy and the cross diffusion term, denoted as $X$, results from the transformation of the transport equation for a turbulent quantity.

The cross diffusion term $X$ can be retained representing the pure transformation equation but in practice, its negation has little effect on results as long as model constants 
are adjusted accordingly. Its influence is limited to regions very close to the wall, particularly where $y^{+}<1$. If the term is retained, extra adjustment is needed in order to avoid a singularity near wall where $k$ approaches zero. The turbulent kinetic energy must be limited in order to avoid this case. Thus, for simplicity, reduced computational effort, and without loss of accuracy, the cross diffusion term is neglected.

The final equation is obtained by inserting the transformed quantity $\zeta=v^{2} / k$ and also omitting the cross diffusion $X$. Thus the $\zeta$ transport equation presented in standard source-sink-diffusion form is

$$
\frac{D \zeta}{D t}=f-\frac{\zeta}{k} \frac{P_{k}}{\rho}+\frac{\partial}{\partial x_{k}}\left[\left(v+\frac{v_{t}}{\sigma_{\zeta}}\right) \frac{\partial \zeta}{\partial x_{k}}\right]
$$

where $\sigma_{\zeta}$ is the turbulent Prandtl number for $\zeta$.

\section{B. $\zeta$ Equation Benefits}

While the transport equations for $\zeta$ and $v^{2}$ are identical mathematically as long as the cross diffusion term is included, Popovac describes the differences between the two which results in enhanced stability and robustness while using the $\zeta$ transport equation as opposed to the $v^{2}$ transport equation. The advantages are described below.

Firstly, the source term in the $\zeta$ equation are better defined throughout the flow field. The $v^{2}$ source term contains the turbulence dissipation $\varepsilon$ which is difficult to reproduce accurately due to the modeled transport equation especially in the near wall region. The non-zero value at the wall accentuates this flow. The source term for the $\zeta$ equation, however, represents the dissipation as function of the turbulent production, $P_{k}$. Capturing this quantity accurately is much easier as long as the turbulent stresses and the mean velocity gradients are represented properly. The value of the turbulent production is also zero at the wall, contrasting the $v^{2}$ source term greatly. 
Another benefit of the $\zeta$ equation can be seen by examining the equilibrium balance of the source and diffusion terms in near wall conditions. In the $v^{2}$ equation, both of the source terms as well as the diffusion term are proportional to $y^{2}$ and therefore must all balance as $y$ approaches zero near the wall. This requires the three terms to balance properly in order to reach a steady state flow condition. Contrastingly, the sink or dissipation source term in the $\zeta$ equation, as discussed earlier, approaches zero at a much higher rate than the both the production source and the diffusion term. Popovac explains that this is increasingly important when segregated solvers, such as FLUENT, are used in which coupling of the variables is delayed to outer iterations.

Yet another benefit is a less stiff boundary condition for the elliptic relaxation equation. If the $\zeta$ transport equation is balanced as $y$ approaches zero (at the wall), it can be determined that the boundary condition for $f$ is

$$
f_{w}=\frac{-2 v \zeta}{y^{2}} \text { as } y \rightarrow 0
$$

Also, under equilibrium circumstances, $\zeta$ is proportional to $y^{2}$ and thus the wall boundary condition is nearly constant. For the $v^{2}$ equation, however, the boundary condition is a function of $y^{4}$. Similar to $f_{w}$ for $\zeta, v^{2}$ is proportional to $y^{4}$ and thus the boundary condition is also nearly constant along the wall. Therefore, no benefit is seen in that respect. The dependence on $y^{2}$ as opposed to $y^{4}$, however, does impose a less stiff condition leading to less grid dependence and therefore a more robust model. Interestingly, this boundary condition has the same form as the turbulent dissipation, $\varepsilon$, wall boundary condition. Noticing this, it is important to recognize that the elliptic relaxation function $f$ can be transformed to obtain a zero wall boundary condition. The transformation is presented below. 


$$
\tilde{f}=f+\left(\frac{\partial \zeta^{1 / 2}}{\partial x_{n}}\right)^{2}
$$

where the second term is an alternate representation for $2 v \zeta / y^{2}$. Popovac claims that this term is a more accurate prediction to the polynomial expansion of $\zeta$ at the wall. This transformation results in the wall boundary condition $\tilde{f}_{w}=0$. This transformation will be applied later for the $f$ transport equation to obtain a more robust model.

This benefit is somewhat lost, however, due to a similar reformulation of the elliptic relaxation equation used in the $v^{2}-f$ model. The reformulation by Lien et al. produces a zero wall boundary condition for $f$ as well. Popovac does, however, claim that the modification results in a less accurate $v^{2}-f$ model.

The last benefit of the $\zeta$ transport equation stems from its mathematical limiting values. For the normalized variable $\zeta$, the values are bounded while for the nonnormalized $v^{2}$, there are no upper bounds. This is evident by applying the Schwarz inequality, $\overline{u_{i} u_{i}}>0$, as well as the definition of the turbulent kinetic energy, $k=\overline{u_{i} u_{i}} / 2$. Using the two aforementioned equations, it can be shown that

$$
\begin{gathered}
\frac{\overline{u u}}{k}+\frac{\overline{v v}}{k}+\frac{\overline{w w}}{k}=2 \\
\text { for } \frac{\overline{u u}}{k} \rightarrow 0 \text { and } \frac{\overline{w w}}{k} \rightarrow 0 \\
0 \leq \frac{\overline{v v}}{k}=\zeta \leq 2
\end{gathered}
$$

These mathematical constraints on $\zeta, 0 \leq \zeta \leq 2$, are even less constraining than the physical constraints. In reality, the normal Reynolds stress components do not reach zero and therefore the equalities on either side of $\zeta$ are not represented in practice. These 
bounds are especially useful for implementation and debugging. With bounded values, gradients are also more easily predicted in a numerical scheme.

In addition to the previously stated bounds, $\zeta$ is also a good measure of anisotropy within the flow field. For isotropic flow, $\overline{u u}=\overline{v v}=\overline{w w}$, applying the Schwarz equality as well as the definition of the turbulent kinetic energy again, $\zeta$ can be written as

$$
\begin{aligned}
& 3 \frac{\overline{v v}}{k}=2 \\
& \zeta=2 / 3
\end{aligned}
$$

This isotropic value is of course ubiquitous with the isotropic state for $v^{2}, v^{2}=2 k / 3$, and provides no numerical benefit. Visually, however, it is easy to see when the flow region departs from the isotropic state, and it is also more apparent what the level of anisotropy is within the region. When departing from $\zeta=2 / 3$ (the isotropic flow condition) towards zero, the wall normal Reynolds stress component approaches zero. When departing towards $\zeta=2$, the wall normal component of the Reynolds stress dominates over the other two turbulent stress components.

\section{Elliptic Relaxation Function}

Durbin initially proposed an elliptic relaxation model in order to model near wall effects. Examining the Reynolds stress model and the importance of properly modeling the pressure fluctuations, individual pressure related terms (the wall reflection, slow and rapid term) are modeled individually. To model these terms, Durbin proposed solving an elliptic equation of the Helmholtz type shown below.

$$
L^{2} \nabla^{2} f_{i j}-f_{i j}=-\frac{R_{i j}+\varepsilon a_{i j}}{k}
$$


where $L$ is the length scale, $f_{i j}$ is an intermediate variable which is related to the pressure rate of strain $R_{i j}$ normally as $R_{i j}=k f_{i j}$. This is introduced in order to enforce proper wall behavior and thus removes the need to model the individual wall reflection portion of the pressure rate of strain. This method thus only requires the modeling of the two remaining pressure effects, the slow and rapid term represented as $R_{i j}=R_{i j, 1}+R_{i j, 2}$ respectively. The rapid part, $R_{i j, 2}$, responds immediately to the change of the mean flow deformation. The slow part, however, $R_{i j, l}$, represents the interaction of fluctuating velocities. These two terms have been modeled in multiple ways.

In anisotropic flow without mean velocity gradients, the pressure rate of strain reduces solely to the slow part and the flow generally returns to an isotropic state. Thus, it was proposed that the slow part could be modeled as a return to isotropy (RI). This model simulates the effect of the pressure fluctuation diminishing turbulence anisotropy.

$$
R_{i j, 1}^{R I}=-C_{1} \varepsilon a_{i j}=-C_{1} \varepsilon\left(\frac{\overline{u_{i} u_{i}}}{k}-\frac{2}{3} \delta_{i j}\right)
$$

The model is a linear model with respect to the anisotropy tensor $a_{i j}$. Speziale et al, however, proposed that the return to isotropy is a non-linear process and thus proposed a non-linear relationship as defined below.

$$
R_{i j, 1}^{S S G}=-\varepsilon\left[C_{1} a_{i j}+C_{1}^{\prime}\left(a_{i k} a_{j k}-\frac{1}{3} \delta_{i j} a_{m n} a_{m n}\right)\right]
$$

where SSG represents the model creators Speziale, Sarkar and Gatski.

The rapid part is modeled through analogy to the slow part and was proposed by Naot et al.

$$
R_{i j, 2}^{I P}=-C_{2}\left(P_{i j}-\frac{2}{3} \delta_{i j} P\right)
$$


where $P_{i j}$ represents the production tensor and $P$ is the production defined as $P_{k} / \rho$. This linear model is commonly referred to as the isotropisation of production (IP) which claims that the rapid part goes against the effects of production in increasing anisotropy.

Again, Speziale et al., proposed an alternate model, coined the quasi-linear form for the rapid part.

$$
\begin{aligned}
R_{i j, 2}^{S S G}= & -C_{2}^{\prime} P a_{i j}+C_{3} k S_{i j}+ \\
& C_{4} k\left(a_{i k} S_{i j}+a_{j k} S_{i k}-\frac{2}{3} \delta_{i j} a_{k l} S_{k l}\right)+ \\
& C_{5}\left(a_{i k} \Omega_{j k}+a_{j k} \Omega_{i k}\right)
\end{aligned}
$$

In the standard $v^{2}-f$ model developed by Durbin, the linear IP model is used for the rapid part. The elliptic relaxation function used in the $\zeta-f$ model has been modified to include the quasi-linear SSG model for the rapid part of the pressure strain term. According to Popovac, this allows the model to better capture stress anisotropy in wall boundary layers. If Eq. 106 is applied to the wall normal stress component, $R_{22,2}$, and assuming that $P_{22}=0$, with the previously defined $\zeta$ equation without the cross diffusion term, the following elliptic relaxation equation is obtained.

$$
L^{2} \frac{\partial^{2} f}{\partial x_{i} \partial x_{i}}-f=\frac{1}{T}\left(C_{1}-1+C_{2}^{\prime} \frac{P}{\varepsilon}\right)\left(\zeta-\frac{2}{3}\right)-\left(\frac{C_{4}}{3}-C_{5}\right) \frac{P}{k}
$$

where it is important to remember that $P$ is the production without density. It is also important to note that the last term can be neglected as the constants determined from the SSG model $\left(C_{4}=0.625\right.$ and $\left.C_{5}=0.2\right)$ produce a coefficient of approximately 0.008 .

Thus after the discussed modifications are made, the $\zeta$ transport equation, $f$ transport equation and also turbulent viscosity are defined as follows.

$$
v_{t}=C_{\mu} \zeta k T
$$




$$
\begin{gathered}
\frac{D \zeta}{D t}=f-\frac{\zeta}{k} \frac{P_{k}}{\rho}+\frac{\partial}{\partial x_{k}}\left[\left(v+\frac{v_{t}}{\sigma_{\zeta}}\right) \frac{\partial \zeta}{\partial x_{k}}\right] \\
L^{2} \frac{\partial^{2} f}{\partial x_{i} \partial x_{i}}-f=\frac{1}{T}\left(C_{1}-1+C_{2}^{\prime} \frac{P_{k}}{\rho \varepsilon}\right)\left(\zeta-\frac{2}{3}\right)
\end{gathered}
$$

where $L$ and $T$ are the length and time scales respectively which are to be defined and the model constants will be summarized in a later section.

\section{Time and Length Scales}

The time and length scales are increasingly important for an elliptic relaxation turbulence model. This is due to the large dependence on accurately capturing the elliptic relaxation function which in turn depends largely on the time and length scales. From a numerical perspective, avoiding singularities is imperative and thus the scales must be limited. This is also true from a physical perspective where turbulent scales cannot drop below Kolmogorov scales. On the upper end, realizability constraints limit both scales. The time and length scales respectively are presented below.

$$
\begin{gathered}
T=\max \left[\min \left(\frac{k}{\varepsilon}, \frac{C_{T}}{\sqrt{6} C_{\mu} S \zeta}\right), C_{\tau}\left(\frac{v}{\varepsilon}\right)^{1 / 2}\right] \\
L=C_{L} \max \left[\min \left(\frac{k^{3 / 2}}{\varepsilon}, \frac{k^{1 / 2}}{\sqrt{6} C_{\mu} S \zeta}\right), C_{\eta}\left(\frac{v^{3}}{\varepsilon}\right)^{1 / 4}\right]
\end{gathered}
$$

where the constants will be summarized later and the strain rate magnitude $S$ is defined as

$$
S=\sqrt{2 S_{i j} S_{i j}}
$$




\section{E. $\zeta_{-} f$ with Zero Wall Boundary Condition}

The $f$ elliptic relaxation equation has been modified further by Popovac in order to obtain a zero wall boundary condition and increase robustness. The transformation, which was discussed previously, is of the same form as the wall boundary condition for the turbulent dissipation.

$$
\tilde{f}=f+\left(\frac{\partial \zeta^{1 / 2}}{\partial x_{n}}\right)^{2}
$$

From this point, the tilde over the transformed $f$ will be omitted. This transformation of the $f$ transport equation not only affects the transport equation being directly transformed but also the $\zeta$ transport equation. The equations can also be adjusted to account for the variability of density. The adjust equations are presented below.

$$
\begin{gathered}
\frac{\partial(\rho \zeta)}{\partial t}+\frac{\partial\left(\rho u_{i} \zeta\right)}{\partial x_{i}}=\rho f-\frac{\zeta}{k}\left[P_{k}+\rho \varepsilon\right]+\frac{\partial}{\partial x_{j}}\left[\left(\mu+\frac{\mu_{t}}{\sigma_{\zeta}}\right) \frac{\partial \zeta}{\partial x_{j}}\right] \\
L^{2} \frac{\partial^{2} f}{\partial x_{i} \partial x_{i}}-f=\frac{1}{T}\left[\left(C_{1}-1+C_{2} \frac{P_{k}}{\rho \varepsilon}\right)\left(\zeta-\frac{2}{3}\right)+\zeta\right]
\end{gathered}
$$

where the constants are to be summarized and the wall boundary condition for $f$ is $f_{w}=0$.

\section{F. ל-f Summary}

The $\zeta-f$ model can now be summarized completely including the two additional transport equations for the turbulent kinetic energy, $k$, and the turbulent dissipation, $\varepsilon$. Slight modifications to the $\varepsilon$ transport equation have also been made which are similar to that of the $v^{2}-f$ model. The final model equations are displayed below.

$$
\mu_{t}=\rho C_{\mu} k \zeta T
$$




$$
\begin{gathered}
\frac{\partial(\rho k)}{\partial t}+\frac{\partial\left(\rho u_{i} k\right)}{\partial x_{i}}=P_{k}-\rho \varepsilon+\frac{\partial}{\partial x_{j}}\left[\left(\mu+\frac{\mu_{t}}{\sigma_{k}}\right) \frac{\partial k}{\partial x_{j}}\right] \\
\frac{\partial(\rho \varepsilon)}{\partial t}+\frac{\partial\left(\rho u_{i} \varepsilon\right)}{\partial x_{i}}=\frac{C_{\varepsilon 1}^{\prime} P_{k}-C_{\varepsilon 2} \rho \varepsilon}{T}+\frac{\partial}{\partial x_{j}}\left[\left(\mu+\frac{\mu_{t}}{\sigma_{\varepsilon}}\right) \frac{\partial \varepsilon}{\partial x_{j}}\right] \\
\frac{\partial(\rho \zeta)}{\partial t}+\frac{\partial\left(\rho u_{i} \zeta\right)}{\partial x_{i}}=\rho f-\frac{\zeta}{k}\left[P_{k}+\rho \varepsilon\right]+\frac{\partial}{\partial x_{j}}\left[\left(\mu+\frac{\mu_{t}}{\sigma_{\zeta}}\right) \frac{\partial \zeta}{\partial x_{j}}\right] \\
L^{2} \frac{\partial^{2} f}{\partial x_{i} \partial x_{i}}-f=\frac{1}{T}\left[\left(C_{1}-1+C_{2} \frac{P_{k}}{\rho \varepsilon}\right)\left(\zeta-\frac{2}{3}\right)+\zeta\right]
\end{gathered}
$$

where $T$ and $L$ are defined as

$$
\begin{gathered}
T=\max \left[\min \left(\frac{k}{\varepsilon}, \frac{C_{T}}{\sqrt{6} C_{\mu} S \zeta}\right), C_{\tau}\left(\frac{v}{\varepsilon}\right)^{1 / 2}\right] \\
L=C_{L} \max \left[\min \left(\frac{k^{3 / 2}}{\varepsilon}, \frac{k^{1 / 2}}{\sqrt{6} C_{\mu} S \zeta}\right), C_{\eta}\left(\frac{v^{3}}{\varepsilon}\right)^{1 / 4}\right]
\end{gathered}
$$

The model constants defined by Popovac are presented below in Table 1.

Table 1. Summary of $\zeta-f$ model constants determined by Popovac

\begin{tabular}{|c|c|c|c|c|c|c|c|c|c|c|c|c|}
\hline $\boldsymbol{\mu}_{\boldsymbol{t}}$ & $\boldsymbol{k}$ & \multicolumn{4}{|c|}{$\boldsymbol{\varepsilon}$} & $\zeta$ & \multicolumn{2}{c|}{$\boldsymbol{f}$} & \multicolumn{2}{|c|}{$\boldsymbol{T}$} & \multicolumn{2}{c|}{$\boldsymbol{L}$} \\
\hline$C_{\mu}$ & $\sigma_{k}$ & $C_{\varepsilon 1}^{\prime}$ & $C_{\varepsilon 1}$ & $C_{\varepsilon 2}$ & $\sigma_{\varepsilon}$ & $\sigma_{\zeta}$ & $C_{1}$ & $C_{2}$ & $C_{T}$ & $C_{\tau}$ & $C_{L}$ & $C_{\eta}$ \\
\hline 0.22 & 1.0 & $C_{\varepsilon 1}(1+0.012) / \zeta$ & 1.4 & 1.9 & 1.3 & 1.2 & 1.4 & 0.65 & 0.6 & 6.0 & 0.36 & 50 \\
\hline
\end{tabular}

These constants will be recalibrated for the current implementation in FLUENT.

Lastly, the boundary conditions at the wall for each of the transport equations are summarized below. 


$$
\begin{gathered}
k_{w}=0 \\
\varepsilon_{w}=\frac{2 v k_{p}}{y_{p}^{2}} \\
\zeta_{w}=0 \\
f_{w}=0
\end{gathered}
$$

where $v$ is the kinematic molecular viscosity and subscripted "p" indicates that those are the values at the cell center in the wall adjacent cell. The value $y_{p}$ is the wall normal distance from the cell center to the wall face center.

\section{G. Calibration of $\zeta-f$ in FLUENT}

The constants that are used within the $v^{2}-f$ model have often been altered since the models first introduction. The $\zeta-f$ model by Popovac utilizes the same constants which have been altered from the $v^{2}-f$ model. The constants used in the $\zeta-f$ model by Popovac were calibrated for its implementation. Similarly, for the implementation in FLUENT, the model constants have been calibrated to better predict turbulent flat plate flow.

The calibration consists of parametrically varying constants which have the most influence on the skin friction coefficient and the boundary layer profile. The goal, of course, is to match the previously discussed power law for the skin friction coefficient and Spaldings formula and Wieghardt data for the boundary layer profile. It was determined that $\sigma_{k}, \sigma_{\varepsilon}, \sigma_{\zeta}, C^{\prime}{ }_{\varepsilon 1}, C_{\varepsilon 1}, C_{\varepsilon 2}, C_{1}, C_{T}$, and $C_{\tau}$ have little basis for change as they have been determined and verified previously. These coefficients have the same value as the Popovac implementation. The length scale coefficients, however, were modified before in order to adjust for the exclusion of the cross diffusion term. Therefore, these coefficients are reexamined for this implementation. Additionally, $C_{\mu}=0.22$ was not modified because its value has been determined based on extensive study by Durbin and 
others. The effect of varying the remaining constants is displayed below in Figure 7 for the skin friction coefficient and Figure 8 for the boundary layer profile. The black colored lines indicate the original model constants as defined by Popovac.
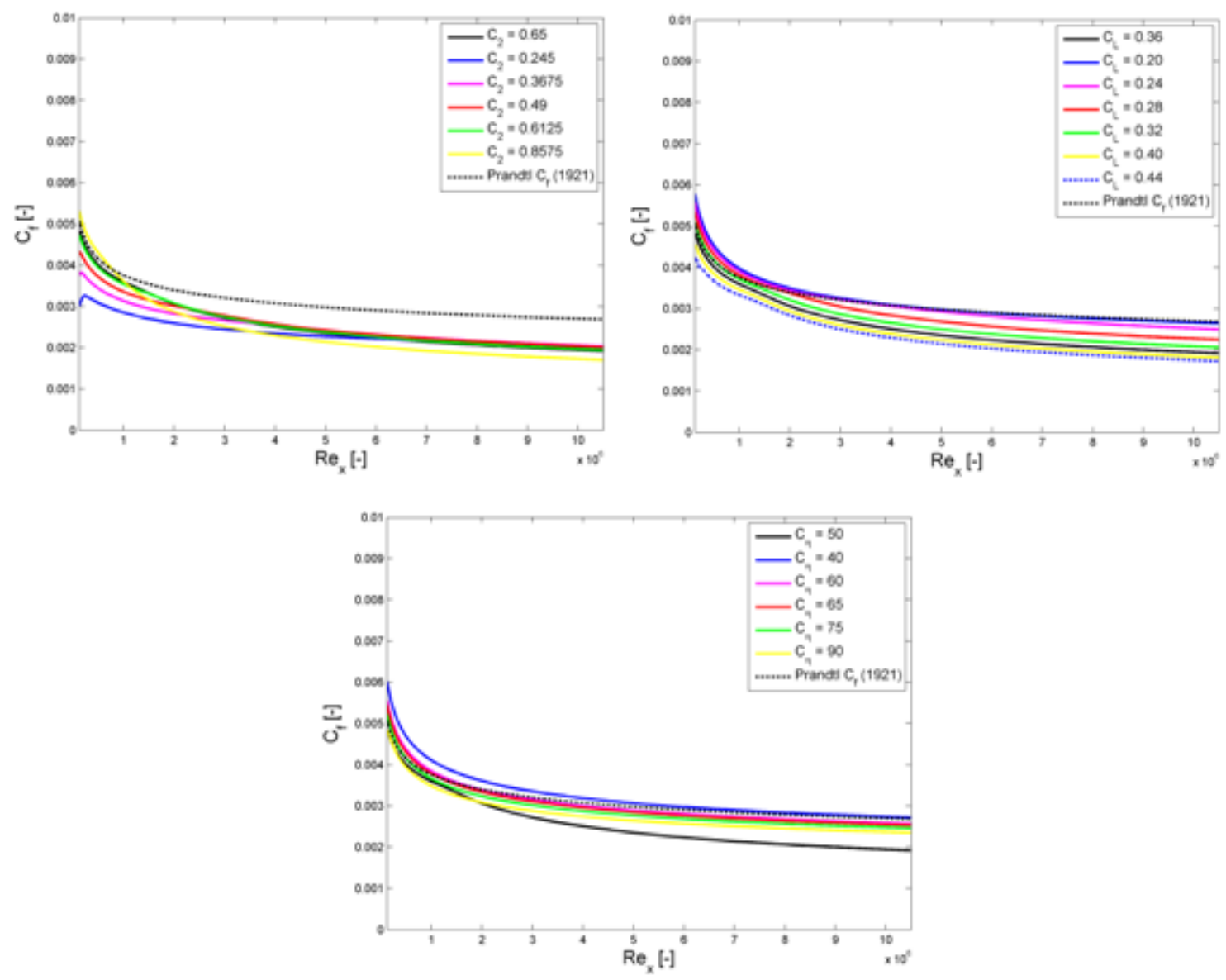

Figure 7. Calibration curves for skin friction coefficient. 

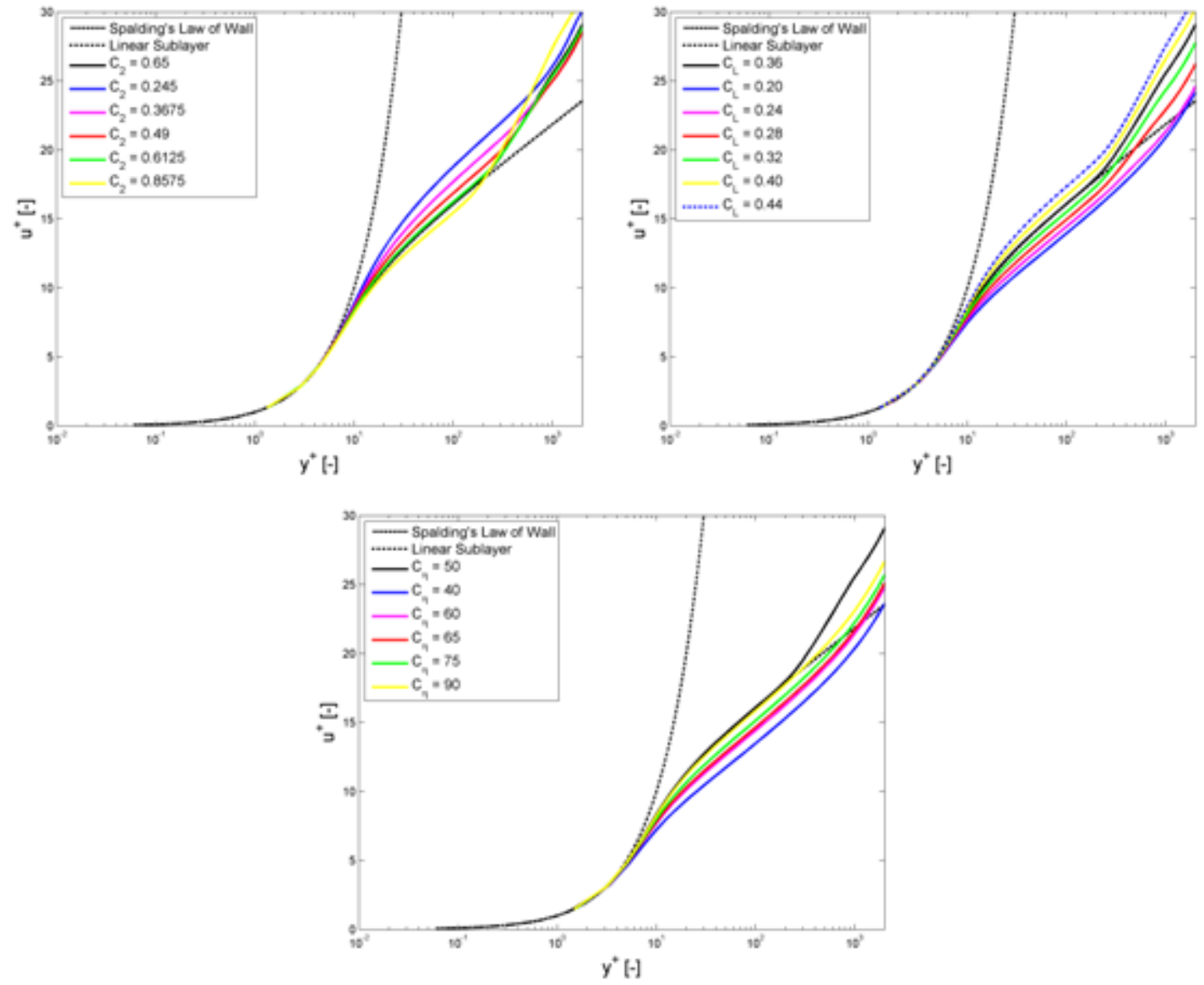

Figure 8. Calibration curves for boundary layer profile.

The calibration was done to try balance the accuracy of the boundary layer profile as well as the skin friction coefficient. The results of the calibration are displayed below in Table 2. Only two constants from the length scale definition were modified while the rest retained their values used in the Popovac model.

Table 2. Calibrated $\zeta-f$ model constants.

\begin{tabular}{|c|c|c|c|c|c|c|c|c|c|c|c|c|}
\hline $\boldsymbol{\mu}_{\boldsymbol{t}}$ & $\boldsymbol{k}$ & \multicolumn{4}{|c|}{$\boldsymbol{\varepsilon}$} & $\zeta$ & \multicolumn{2}{c|}{$\boldsymbol{f}$} & \multicolumn{2}{|c|}{$\boldsymbol{T}$} & \multicolumn{2}{c|}{$\boldsymbol{L}$} \\
\hline$C_{\mu}$ & $\sigma_{k}$ & $C_{\varepsilon 1}^{\prime}$ & $C_{\varepsilon 1}$ & $C_{\varepsilon 2}$ & $\sigma_{\varepsilon}$ & $\sigma_{\zeta}$ & $C_{1}$ & $C_{2}$ & $C_{T}$ & $C_{\tau}$ & $C_{L}$ & $C_{\eta}$ \\
\hline 0.22 & 1.0 & $C_{\varepsilon 1}(1+0.012) / \zeta$ & 1.4 & 1.9 & 1.3 & 1.2 & 1.4 & 0.65 & 0.6 & 6.0 & 0.28 & 65 \\
\hline
\end{tabular}




\section{Algebraic Structure Based Model}

The ASBM was formulated from the eddy axis concepts developed by Kassinos and Reynolds. The eddy axis formulation computes the structure tensors from a statistical average of hypothetical turbulent eddies. The eddies are characterized by its axis vector, vortical and jetal fluctuating velocity components, and a measure of cross-sectional axisymmetry. These quantities are represented by the eddy axis tensor $a_{i j}$, the eddy-jetal parameter $\varphi$, the eddy-helical vector $\gamma_{k}$, and the eddy-flattening scalar $\chi$ and eddy-

flattening tensor $F_{i j}{ }^{r}$ respectively. The eddy-axis tensor is the energy weighted average of normalized eddy axes. The eddy-jetal parameter is the fraction of energy contained in the jetal mode while the eddy-helical vector is the correlation of the vortical and jetal motions due to rotation. The eddy-flattening scalar and tensor measure the magnitude and direction of axisymmetric energy distribution. The ASBM is an algebraic model of these quantities where the Reynolds stress tensor is calculated from these values.

\section{A. Structure Tensors}

The turbulence tensors are $R_{i j}$, the Reynolds stress tensor, $D_{i j}$, the dimensionality structure tensor, $F_{i j}$, the circulicity structure tensor. $D_{i j}$ and $F_{i j}$ contain turbulent structure information not present in the Reynolds stress. For the case of homogenous turbulence, the contractions of the tensors are all twice the turbulent kinetic energy, or $R_{i i}=D_{i i}=F_{i i}$ $=q^{2}=2 k$. Normalized structure tensors can then be defined as

$$
r_{i j} \equiv R_{i j} / q^{2}, d_{i j} \equiv D_{i j} / q^{2}, f_{i j} \equiv F_{i j} / q^{2}
$$


It can also be shown that in homogeneous turbulence, only two structure tensors are linearly independent. Therefore,

$$
r_{i j}+d_{i j}+f_{i j}=\delta_{i j}
$$

This relationship further affirms that modeling with only one of these structures, as is done in LEVMs, would be difficult.

\section{B. Eddy Properties}

In order to properly define turbulent eddies, each eddy is thought of as separate particles from the fluid. This visualization is similar to the Particle Representation Method (PRM) also develop by Kassinos and Reynolds. Each of the particles in the eddy share a eddy-axis direction vector, $\vec{a}$, while each has its own velocity vector, $\vec{V}$, and a gradient vector, $\vec{n}$, perpendicular to both $\vec{a}$ and $\vec{V}$. There is a also a stream function vector, $\vec{s}$, perpendicular to $\vec{V}$ and $\vec{n}$ forming a mutually orthogonal vector triad. As previously mentioned, the eddy can also be flattened. This flattening accounts for asymmetry in the eddy and can be thought of as a axisymmetric distribution of an eddy's kinetic energy being either partially or fully flattened into elliptic or sheet form respectively. This flattening is defined by a flattening direction, $\vec{b}$, and also a flattening intensity.

These conceptual eddies are the basis of the ASBM. Within a turbulent field there are many of these conceptual eddies which define field. If the ensemble of eddies is averaged throughout the field, the result would be a representation of the entire field. This ensemble-average, denoted below by angle brackets, is used to represent both the eddy-axis tensor and the eddy-flattening tensor. 
The eddy-axis tensor is the energy weighted average direction cosine tensor of the eddy axes.

$$
a_{i j}=\frac{1}{q^{2}}\left\langle V^{2} a_{i} a_{j}\right\rangle
$$

where $q^{2}$ is again equal to twice the turbulent kinetic energy. The eddy axis tensor is determined by the mean deformation and generally aligns with the direction of positive mean strain. Both mean and frame rotation only rotate the eddy axis tensor kinematically and not dynamically.

The eddy flattening tensor, similarly defined as the eddy-axis tensor, is the ensemble average direction cosine tensor of the flattening vector,

$$
b_{i j}=\frac{1}{q^{2}}\left\langle V^{2} b_{i} b_{j}\right\rangle
$$

In order to more easily represent the eddies, four parameters are defined that represent the previously mentioned eddy properties. These parameters are the eddy jetting parameter, $\varphi$, the eddy helix vector, $\gamma$, the eddy flattening parameter, $\chi$, and $\beta$ which is indicative of the degree of correlation between the fluctuating velocities in different directions. The eddy jetting parameter represents the energy present in the jetal mode of the eddy. Consequently, $(1-\varphi)$ represents the energy present in the vortical mode of the eddy. When the deformations representing the eddy are irrotational, the eddy jetting parameter is zero and thus the eddies are purely vortical. In this case, the eddy-axis tensor also coincides with the circulicity tensor, $f_{i j}$, previously defined. Rotation, however, creates eddies with energy in the jetal mode. For shear in a fixed frame, the eddy jetal parameter thus approaches one. 
The eddy helix vector represents the correlation between the jetal and vortical modes of motion of the eddy. If the eddy helix vector components are equal to zero, it corresponds to purely vortical or purely jetal turbulence. Typically it is aligned with the total vorticity vector. The shear stress level in the turbulence field is determined mainly from the helix vector.

The eddy flattening parameter represents any asymmetry within the eddy. A nonaxisymmetric eddy is thus flattened. Irrotational deformation in a fixed frame creates eddies that are not flattened while the presence of rotation flattens eddies into planes perpendicular to the rotation. Ultimately, these three parameters along with the eddy-axis tensor and eddy flattening tensor are what are modeled to represent the turbulent field.

\section{Model Formulation}

The structure tensors are related to the parameters of the hypothetical eddy field presented by the eddy axis concept as shown below

$$
\begin{aligned}
& r_{i j}=\frac{\overline{u_{i}^{\prime} u_{j}^{\prime}}}{2 k}=(1-\phi) \frac{1}{2}\left(\delta_{i j}-a_{i j}\right)+\phi a_{i j}+ \\
&(1-\phi) \chi\left[\frac{1}{2}\left(1-a_{n m} b_{m n}\right) \delta_{i j}-\frac{1}{2}\left(1+a_{n m} b_{m n}\right) a_{i j}-b_{i j}+a_{i n} b_{n j}+a_{j n} b_{n i}\right]+ \\
&\left(\frac{-\gamma \Omega_{k}^{\mathrm{T}}}{\Omega^{\mathrm{T}}}\right)\left(\varepsilon_{i p r} a_{p j}+\varepsilon_{j p r} a_{p i}\right) \times\left\{\frac{1}{2}\left[1-\chi\left(1-a_{n m} b_{m n}\right)\right] \delta_{k r}+\chi b_{k r}-\chi a_{k n} b_{n r}\right\} \\
& d_{i j}= \frac{1}{2}\left(\delta_{i j}-a_{i j}\right)+ \\
& \chi\left[-\frac{1}{2}\left(1-a_{n m} b_{m n}\right) \delta_{i j}+\frac{1}{2}\left(1+a_{n m} b_{m n}\right) a_{i j}+b_{i j}-\left(a_{i n} b_{n j}+a_{j n} b_{n i}\right)\right] \\
& f_{i j}=\delta_{i j}-r_{i j}-d_{i j}
\end{aligned}
$$


where $a_{i j}$ is the homogenous eddy axis tensor. It is important to note that under irrotational mean deformation, eddies are purely vortical and thus $\phi$ is zero. Similarly, shear produces jetal eddies implying flows with rapid distortion have values of $\phi$ closer to one. The strained eddy axis tensor is defined as

$$
a_{i j}^{*}=\frac{1}{3} \delta_{i j}+\frac{\left(S_{i k}^{*} a_{k j}^{*}+S_{j k}^{*} a_{k i}^{*}-\frac{2}{3} S_{m n}^{*} a_{n m}^{*} \delta_{i j}\right) \tau}{a_{0}+2 \sqrt{\tau^{2} S_{k p}^{*} S_{k q}^{*} a_{p q}^{*}}}
$$

where $S_{i j}^{*}=S_{i j}-S_{k k} \delta_{i j} / 3$ is the traceless strain rate tensor. The time scale of turbulence, $\tau$, is calculated based on the chosen scalar transport equations used with the ASBM. The "slow" constant, $a_{0}$ is equal to 1.6. The homogeneous eddy axis tensor is then obtained by applying a rotation transformation to the strained eddy-axis tensor as shown below

$$
a_{i j}=H_{i k} H_{j l} a_{k l}^{*} \quad H_{i j}=\delta_{i j}+h_{1} \frac{\Omega_{i j}}{\sqrt{\Omega_{p q} \Omega_{p q}}}+h_{2} \frac{\Omega_{i k} \Omega_{k j}}{\Omega_{p q} \Omega_{p q}}
$$

where $\Omega_{p q}$ is the mean rotation rate tensor. The orthonormality conditions for $H$, $H_{k i} H_{k j}=\delta_{i j}$, requires that

$$
h_{1}=\sqrt{2 h_{2}-h_{2}^{2} / 2}
$$

where $h_{2}$ is calculated based on rapid distortion theory for combined homogeneous plane strain and rotation.

$$
h_{2}=\left\{\begin{array}{ll}
2-2 \sqrt{\frac{1}{2}(1+\sqrt{1-r})} & \text { if } r \leq 1 \\
2-2 \sqrt{\frac{1}{2}(1-\sqrt{1-1 / r})} & \text { if } r \geq 1
\end{array} \quad r=\frac{a_{p q} \Omega_{q r} S_{r p}^{*}}{S_{k n}^{*} S_{n m}^{*} a_{m k}}\right.
$$

The flattening tensor, $b_{i j}$, is given by

$$
b_{i j}=\frac{\left(\Omega_{i}+C_{b} \Omega_{i}^{f}\right)\left(\Omega_{j}+C_{b} \Omega_{j}^{f}\right)}{\left(\Omega_{k}+C_{b} \Omega_{k}^{f}\right)\left(\Omega_{k}+C_{b} \Omega_{k}^{f}\right)}, \quad C_{b}=-1.0
$$


where $\Omega_{i}$ is defined as the mean rotation vector, and $\Omega_{i}^{f}$ is defined as the frame rotation rate vector.

The structure parameters which partially define the normalized Reynolds stress tensor and the normalized dimensionality tensor, $\phi, \chi, \gamma$, are modeled within a threedimensional space defined by the ratio of mean rotation to mean strain, $\eta_{m}$, the ratio of frame rotation to mean strain, $\eta_{f}$, and the mean strain, $a^{2}$. The mean parameters are defined as follows,

$$
\begin{gathered}
\eta_{m} \equiv \sqrt{\frac{\hat{\Omega}_{m}^{2}}{\hat{S}^{2}}} \\
\eta_{f}=\eta_{m}-\operatorname{sign}(X) \sqrt{\frac{\hat{\Omega}_{T}^{2}}{\hat{S}^{2}}} \\
a^{2} \equiv a_{p q} a_{p q}
\end{gathered}
$$

where $\hat{\Omega}_{m}^{2}$ represents the magnitude of rotation, $\hat{\Omega}_{T}^{2}$ represents the magnitude of the total rotation, and $\hat{S}^{2}$ represents the magnitude of strain. Definitions of these magnitude representations are shown below.

$$
\begin{gathered}
\hat{\Omega}_{m}^{2} \equiv-a_{i j} \Omega_{i k} \Omega_{k j} \\
\hat{\Omega}_{T}^{2} \equiv-a_{i j} \Omega_{i k}^{T} \Omega_{k j}^{T} \\
\hat{S}^{2} \equiv-a_{i j} S_{i k} S_{k j} \\
X \equiv a_{i j} \Omega_{i k}^{T} S_{k j}
\end{gathered}
$$

The parameters are defined in a generic $a^{2}$ plane, along the $\eta_{m}$ and $\eta_{f}$ directions. They were then sensitized to the anisotropy of the turbulence along the $a^{2}$ direction. During formulation, modeling was meant to match 2D RDT solutions. Two main RDT 
solution lines were utilized in order to provide regions on the generic $a^{2}$ plane to interpolate and extrapolate from. These two states are shear and plane strain represented by $\eta_{m}=1$ and $\eta_{m}=0$ respectively. The interpolation or extrapolation depends on the flow deformation location in the generic plane in terms of $\eta_{m}$ and $\eta_{f}$. The equations for the known states at $\eta_{m}=1$ and $\eta_{m}=0$ are described below where subscripts of "1" denote the shear state and subscripts of " 0 " denote the plane strain state.

The modeling equations for $\eta_{m}=1$ are displayed below.

If $\eta_{f}<0$,

$$
\phi_{1}=\frac{\eta_{f}-1}{3 \eta_{f}-1}, \quad \beta_{1}=\frac{1}{1-b_{0} \frac{\eta_{f}}{\left(1-a^{2}\right)}\left(1+\sqrt{\left(a^{2}-\frac{1}{3}\right)}\right)}, \quad \chi_{1}=\frac{1}{2} \beta_{1}
$$

If $0<\eta_{f}<1$,

$$
\phi_{1}=\left(1-\eta_{f}\right), \quad \beta_{1}=1, \quad \chi_{1}=\frac{1}{2}+\frac{1}{2}\left(1-\frac{\left(1-\eta_{f}\right)^{2}}{1+b_{1} \eta_{f} /\left(1-a^{2}\right)}\right)
$$

If $\eta_{f}>1$,

$$
\phi_{1}=\frac{\eta_{f}-1}{3 \eta_{f}-1}, \quad \beta_{1}=\frac{1}{1+b_{2} \frac{\left(\eta_{f}-1\right)}{\left(1-a^{2}\right)} \eta_{f} \sqrt{a^{2}-\frac{1}{3}}}, \quad \chi_{1}=1-\frac{\left(1-\beta_{1}\right)\left(\eta_{f}-1\right)}{\left(1-a^{2}\right)+\left(\eta_{f}-1\right)}
$$

where

$$
b_{0}=1.0 \quad b_{1}=100 \quad b_{2}=0.8
$$

The modeling equations for $\eta_{m}=0$ are displayed below.

If $\eta_{f} \leq \sqrt{3} / 4$,

$$
\phi_{0}=0.145\left[\frac{\left(2 \eta_{f}\right)^{2}}{3 / 4}-\left(\frac{\left(2 \eta_{f}\right)^{2}}{3 / 4}\right)^{9}\right]
$$




$$
\begin{gathered}
\beta_{0}=1 \\
\chi_{0}=-\left[0.342 \frac{\left(2 \eta_{f}\right)^{2}}{3 / 4}+(1-0.342)\left(\frac{\left(2 \eta_{f}\right)^{2}}{3 / 4}\right)^{6}\right]
\end{gathered}
$$

Else, if $\eta_{f}>\sqrt{3} / 4$,

$$
\begin{gathered}
\beta_{0}=\left[1+b_{3} \frac{\left(\eta_{f}-\sqrt{3} / 4\right)}{\left(1-a^{2}\right)} \eta_{f} \sqrt{a^{2}-\frac{1}{3}}\right]^{-1} \\
\chi_{0}=-\beta_{0} \\
\phi_{0}=\left(1-\beta_{0}\right) / 3
\end{gathered}
$$

where $b_{3}=1.0$.

For values between $\eta_{m}=1$ and $\eta_{m}=0$, an interpolation is used as specified previously. The region between the shear and plain strain states represents a trapezoidal region where turbulence grows. In order to simplify interpolation, this trapezoidal region is transformed into a rectangular region with the following transformation.

$$
\eta_{*} \equiv-\eta_{m}+\left[4 / \sqrt{3}+(2-4 / \sqrt{3}) \eta_{m}\right] \eta_{f}
$$

After this transformation, the structure parameters are interpolated along lines of constant $\eta_{*}$. The functions for edges of this interpolation utilize an adjusted $\eta_{*}$ to ensure their limits are maintained. The adjusted $\eta_{*}$ for the $\eta_{m}=1$ and $\eta_{m}=0$ lines are defined as follows.

$$
\begin{gathered}
\eta_{1^{*}}=\frac{\left(1+\eta_{*}\right)}{2} \\
\eta_{0^{*}}=\sqrt{3 \eta^{2} / 16}
\end{gathered}
$$

The interpolation is then performed based on these new quantities. 


$$
\begin{aligned}
& \phi^{*}=\phi_{0}\left(\eta_{0^{*}}\right)+\left[\phi_{1}\left(\eta_{1^{*}}\right)-\phi_{0}\left(\eta_{0^{*}}\right)\right] \eta_{m}^{2} \\
& \beta^{*}=\beta_{0}\left(\eta_{0^{*}}\right)+\left[\beta_{1}\left(\eta_{1^{*}}\right)-\beta_{0}\left(\eta_{0^{*}}\right)\right] \eta_{m}^{2} \\
& \chi^{*}=\chi_{0}\left(\eta_{0^{*}}\right)+\left[\chi_{1}\left(\eta_{1^{*}}\right)-\chi_{0}\left(\eta_{0^{*}}\right)\right] \eta_{m}^{4}
\end{aligned}
$$

where the structure parameters are functions of these interpolation quantities.

The extrapolation for the region where $\eta_{m}>1$ requires the decay of turbulence. While there is no exact steady state solution in this region, a limiting state is still reached. The algebraic model utilizes this limiting state and decouples the effect on turbulence that the large rotation causes. Thus the structure parameters are driven down closer to zero. The structure parameters are extrapolated as follows.

$$
\begin{gathered}
\phi^{*}=1 / 3+\frac{\left(\phi_{1}\left(\eta_{f}\right)-1 / 3\right)}{\left.1+\left(\eta_{m}-1\right) /\left(1-a^{2}\right)\right)} \\
\beta^{*}=\frac{\left(\beta_{1}\left(\eta_{f}\right)-1 / 3\right)}{\left.1+\left(\eta_{m}-1\right) /\left(1-a^{2}\right)\right)} \\
\chi^{*}=\frac{\left(\chi_{1}\left(\eta_{f}\right)-1 / 3\right)}{\left.1+\left(\eta_{m}-1\right) /\left(1-a^{2}\right)\right)}
\end{gathered}
$$

where the structure parameters defined at $\eta_{m}=1$ are functions of $\eta_{f}$.

Now these interpolated or extrapolated quantities need to be sensitized to the actual $a^{2}$ plane in which they lie. Recall that before the structure scalars were defined for a generic $a^{2}$ plane. While $\beta$ and $\chi$ have been sensitized to $a^{2}$ in some form, $\phi$ requires a more elaborate transformation.

$$
\phi=\phi^{*}\left(\frac{\left(\eta_{m}-\eta_{f}\right)^{2}}{\left(\eta_{m}-\eta_{f}\right)^{2}+\left(1-a^{2}\right)^{2}}\right)\left(\frac{\left|\eta_{m}-\eta_{f}\right| \sqrt{\frac{3}{2}\left(a^{2}-\frac{1}{3}\right)}}{\left|\eta_{m}-\eta_{f}\right| \sqrt{\frac{3}{2}\left(a^{2}-\frac{1}{3}\right)}+p_{0}\left(1-a^{2}\right)}\right)
$$




$$
\begin{gathered}
\beta=\beta^{*} \\
\chi=\chi^{*}\left[\frac{3}{2}\left(a^{2}-\frac{1}{3}\right)\right]^{p_{1}}
\end{gathered}
$$

where

$$
p_{0}=1.3 \quad p_{1}=1.0
$$

The helix vector, $\gamma_{k}$, is then defined by the structure parameters. It is aligned with the total rotation vector as shown below.

$$
\gamma_{k}=\gamma \frac{\Omega_{k}^{T}}{\sqrt{\Omega_{k}^{T} \Omega_{k}^{T}}}
$$

where $\gamma$ is modeled based on 2D RDT solutions.

$$
\gamma=\beta \sqrt{\frac{2 \phi(1-\phi)}{1+\chi}}
$$

Near wall, however, the model must be adjusted. The act of driving velocity down toward zero by the act of viscous forces reorients the velocity vectors into planes parallel to the wall. The velocity component normal to the wall is driven down much faster than the velocity component tangential to the wall. Thus, it is postulated that eddy orientation will be parallel to the wall in near wall conditions. The wall blocking is modeled through a modified projection operator which is then applied to the tensor that is blocked. The projection is based on the proximity to a wall. A new homogeneous eddy axis tensor, $a_{i j}{ }^{h}$, is computed based on the previous and the effective wall blockage. The free stream $a_{i j}$ is partially projected onto planes parallel to the wall to formulate new wall blocked eddy axis tensor.

$$
a_{i j}=H_{i k} H_{j l} a_{k l}^{h}, \quad H_{i k}=\frac{1}{D_{a}}\left(\delta_{i k}-B_{i k}\right), \quad D_{a}^{2}=1-\left(2-B_{k k}\right) a_{m n}^{h} B_{n m}
$$


where the scalar $D_{a}{ }^{2}$ is formulated so that the trace of $a_{i j}$ remains unity. In the formulation of $D_{a}{ }^{2}$, the trace of the blockage tensor was used as opposed to the second derivative in the wall normal direction for simplicity.

The blockage tensor $B_{i j}$ gives the strength and direction of the projection onto the wall. The blockage tensor is calculated as follows

$$
B_{i j}=\frac{\Phi,,_{i} \Phi,_{j}}{\Phi,,_{k} \Phi_{,_{k}}} \Phi \text { if } \Phi,_{k} \Phi,_{k}>0
$$

where the blocking parameter, $\Phi$, is 1 and $\Phi_{,_{\mathrm{n}}}=0$ solid boundaries where $x_{n}$ is the wallnormal direction. If $\Phi_{, k} \Phi_{, k}$ is zero then the blockage tensor is defined as the zero matrix. The blockage parameter is calculated using a modified Helmoltz equation displayed below.

$$
\begin{gathered}
L^{2} \frac{\partial^{2} \Phi}{\partial x_{k} \partial x_{k}}=\Phi \\
\Phi=1 \quad \text { at solid boundaries } \\
\Phi,_{n} \equiv \frac{\partial \Phi}{\partial x_{n}}=0 \quad \text { at open boundaries }
\end{gathered}
$$

The definition of $L$ was inspired by Durbin and is related to turbulent length scales and Kolmogorov length scales. Multiple definitions fitting this criterion have been attempted but the most stable was proposed by Radhakrishnan,

$$
L=C_{L} \max \left(\frac{k^{3 / 2}}{\varepsilon}, C_{v} \sqrt[4]{\frac{v^{3}}{\varepsilon}}\right)
$$

where $C_{L}=0.80$ and $C_{v}=0.17$. The latter constant, however, has a large effect on the boundary layer profile produced by the model and may be adjusted accordingly. 
The structure parameters are also affected by wall blockage and must be adjusted to reflect the reorientation of eddies. The blocked parameters are defined as functions of their unblocked or homogeneous, denoted by a superscript "h", quantities and the trace of the blockage tensor. Again, the trace of the blockage tensor is used as opposed to its second derivative in the wall-normal direction for simplicity.

$$
\begin{gathered}
\phi=1+\left(\phi^{h}-1\right)\left(1-B_{k k}\right)^{2} \\
\gamma=\gamma^{h}\left(1-B_{k k}\right)
\end{gathered}
$$

The eddy flattening parameter, $\chi$, is unaffected by wall blockage.

\section{ASBM Implementation}

The Algebraic Structure Based Model was implemented in C using a NewtonRaphson method with line search as well as step limiters for both the strained and homogeneous eddy-axis tensor. The tensors to be solved are symmetric and thus are treated as a vector with length six as opposed to nine. The basic Newton-Raphson method is summarized below.

$$
\begin{gathered}
\mathrm{F}(\mathrm{x})=0 \\
\mathrm{x}_{\text {new }}=\mathrm{x}_{\text {old }}+\delta \mathrm{x}
\end{gathered}
$$

where

$$
\delta \mathrm{x}=-\mathrm{J}^{-1} \cdot \mathrm{F}
$$

Here $\mathrm{J}$ is the Jacobian matrix which is computed analytically. The Newton step, $\delta \mathrm{x}$, should decrease the magnitude of $\mathrm{F}$ as it is the descent direction. Thus, try to minimize

$$
f=\frac{1}{2} \mathrm{~F} \cdot \mathrm{F}
$$


It is possible, however, for the step size to be too large resulting in the next guess being further from the solution than the last. This minimization can find local minima as opposed to the global minimum. Instead, a better strategy is to impose the following.

$$
\nabla f \cdot \delta \mathrm{x}=-\mathrm{F} \cdot \mathrm{F}<0
$$

The solution strategy is to calculate the Newton step and attempt the full step. If the full step does not decrease $f$ and satisfy the inequality, then backtracking along the Newton direction is required until an acceptable step size is proposed. In essence,

$$
\mathrm{x}_{\text {new }}=\mathrm{x}_{\text {old }}+\lambda(\delta \mathrm{x}) \quad 0<\lambda \leq 1
$$

Here $\lambda$ is the backtracking fraction which is determined to decrease $f$.

An additionally step size limiter is imposed by the physical limits of the eddy-axis tensor. The eddy-axis tensor requires

$$
\frac{1}{3} \leq a_{i j} a_{j i} \leq 1
$$

This imposes a limit on $\lambda$ based on $\mathrm{x}_{\text {old }}$ and the Newton step. This limit can be expressed quadratic with respect to $\lambda$ as follows,

$$
\begin{gathered}
a \lambda^{2}+b \lambda+c \leq 1 \\
a=\delta \mathrm{x}_{i} \delta \mathrm{x}_{i} \\
b=2 \mathrm{x}_{\text {old }, i} \delta \mathrm{x}_{i} \\
c=\mathrm{x}_{\text {old }, i} \mathrm{x}_{\mathrm{old}, i}
\end{gathered}
$$

keeping in mind that the full length vector must be used (vector length nine). Thus the limit is defined as

$$
\lambda \leq \frac{-b+\sqrt{b^{2}-4 a(c-1)}}{2 a}
$$

This is then imposed as the maximum step size which in practice is almost always much larger than 1. The first step size taken is thus the smaller of the two. 


\section{E. ASBM Verification}

The ASBM was verified against the previous work of Haire and Langer. Verification of the model described in the sections previous is compared against the work of Langer. Haire developed a model based on the same eddy-axis tensor for free shear flows which has been implemented as well. Verification against his work strengthens the verification of the eddy-axis tensor solutions.

Homogeneous solutions were computed for the following flow types: Axisymmetric Contraction (AXC), Axisymmetric Expansion (AXE), Plane Strain (PS), and Shear (SH). The solutions were computed with and without frame rotation. The time scale was also varied to show the models response. In the case of comparison against Haire's results, the non-dimensionalized tensors computed by the model are compared against the strain rate magnitude multiplied by the time scale where the strain rate magnitude is defined as

$$
S=\sqrt{S_{i j} S_{j i}}
$$

Note that this definition varies from that being used in other sections of this paper. For comparisons against Langer's results, they are compared against a dummy variable $\Gamma$ which can be considered as the largest magnitude of the strain rate tensor. The results of the implemented models as well as the previous works are displayed.

\section{Axisymmetric Contraction (AXC)}

The mean deformation for axisymmetric contraction is given by

$$
\begin{aligned}
S_{i j}=\left(\begin{array}{ccc}
1 & 0 & 0 \\
0 & -1 / 2 & 0 \\
0 & 0 & -1 / 2
\end{array}\right), \Omega_{i j} & =\left(\begin{array}{lll}
0 & 0 & 0 \\
0 & 0 & 0 \\
0 & 0 & 0
\end{array}\right), \Omega_{i j}^{f}=\left(\begin{array}{ccc}
0 & 0 & 0 \\
0 & 0 & \Omega^{f} \\
0 & -\Omega^{f} & 0
\end{array}\right) \\
S & =\sqrt{\frac{3}{2}}, \Gamma=1
\end{aligned}
$$


In the Haire model, the value of the slow constant is set to 2. Different levels of frame rotation are introduced as well where $\Omega^{f}=S$ and $\Omega^{f}=2 S$. Figure 9 below displays the comparison of the current implementation and Haire's results. Figure 9 also shows the comparison including frame rotation. The difference in results is negligible.

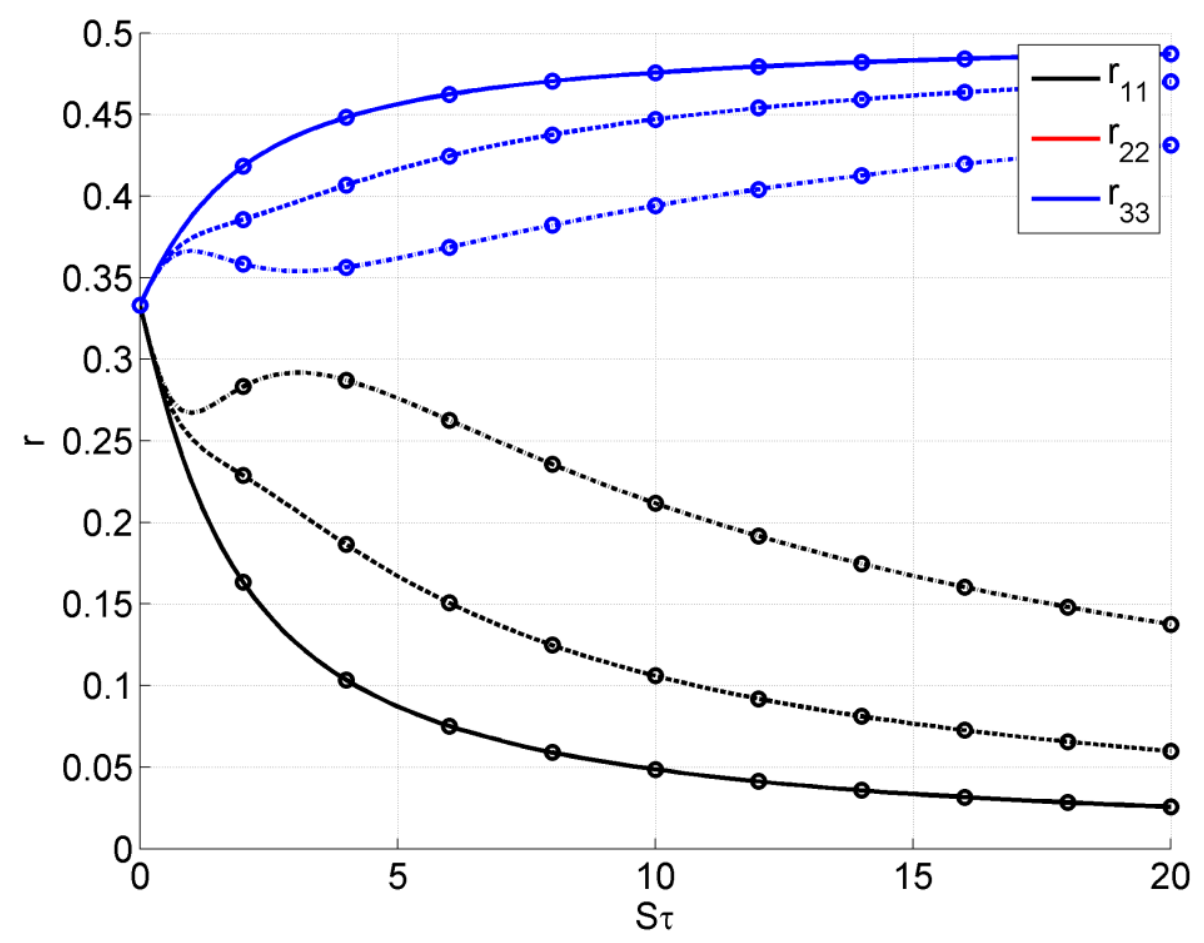

Figure 9. Free shear flow ASBM results under AXC deformation with no frame rotation $\boldsymbol{\Omega}^{f}=0$ (solid line), $\Omega^{f}=S$ (dashed line), $\Omega^{f}=2 S$ (dashed-dot line) compared with Haire's results (o).

The Langer implementation compares the value with the slow constant kept at 1.6. When frame rotation is included, the magnitutde is $\Omega^{f}=2 \Gamma$. Figure 10 displays $b_{i j}=r_{i j}-$ $\delta_{i j} / 3$ compared against the non-dimensionalized strain magnitude. The results are nearly identical. 


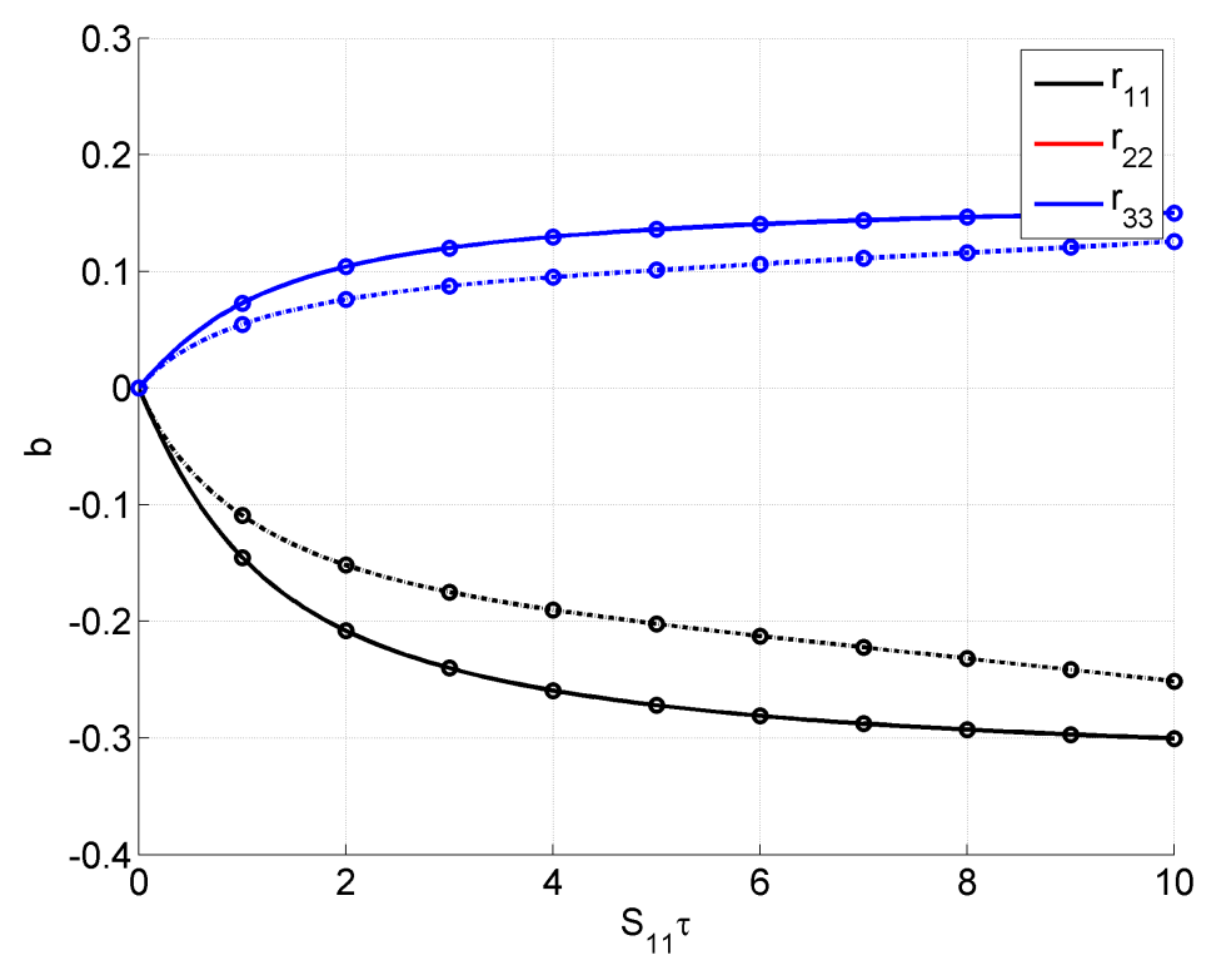

Figure 10. Wall bounded ASBM results under AXC deformation with no frame rotation $\Omega^{f}=0$ (solid line), and $\Omega^{f}=2 \Gamma$ (dashed-dot line) compared with Langer's results (o).

2. Axisymmetric Expansion (AXE)

The mean deformation for axisymmetric expansion is given by

$$
\begin{aligned}
S_{i j}=\left(\begin{array}{ccc}
-1 & 0 & 0 \\
0 & 1 / 2 & 0 \\
0 & 0 & 1 / 2
\end{array}\right), \Omega_{i j} & =\left(\begin{array}{ccc}
0 & 0 & 0 \\
0 & 0 & 0 \\
0 & 0 & 0
\end{array}\right), \Omega_{i j}^{f}=\left(\begin{array}{ccc}
0 & 0 & 0 \\
0 & 0 & \Omega^{f} \\
0 & -\Omega^{f} & 0
\end{array}\right) \\
S & =\sqrt{\frac{3}{2}}, \Gamma=1
\end{aligned}
$$

In the Haire model, the value of the slow constant is set to 2. Different levels of frame rotation are introduced as well where $\Omega^{f}=S$ and $\Omega^{f}=2 S$. Figure 11 below displays the comparison of the current implementation and Haire's results. Figure 11 also shows the comparison including frame rotation. The difference in results is negligible. 


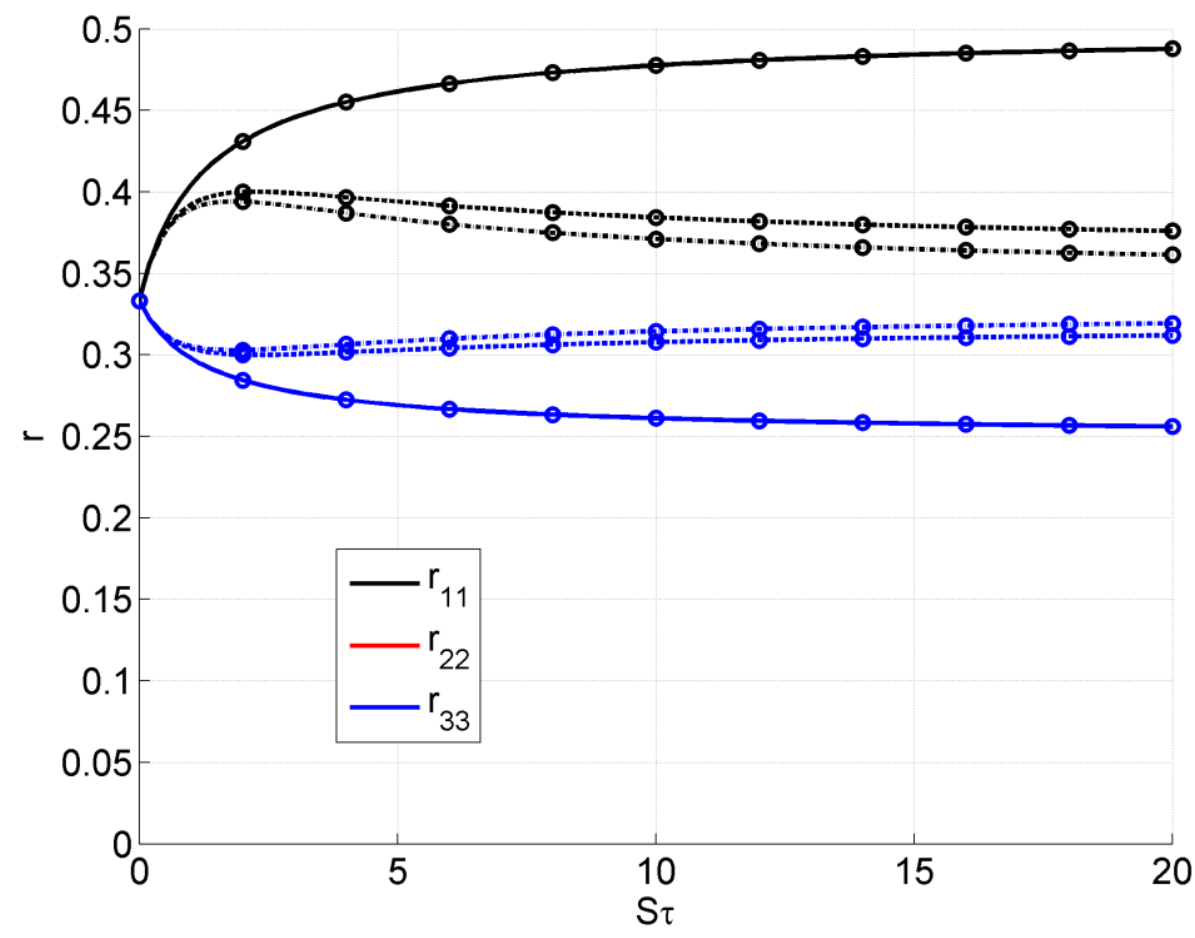

Figure 11. Free shear flow ASBM results under AXE deformation with no frame rotation $\Omega^{f}=0$ (solid line), $\Omega^{f}=S$ (dashed line), $\Omega^{f}=2 S$ (dashed-dot line) compared with Haire's results (o).

The Langer implementation compares the value with the slow constant kept at 1.6. When frame rotation is included, the magnitutde is $\Omega^{f}=2 \Gamma$. Figure 12 displays $b_{i j}=r_{i j}-$ $\delta_{i j} / 3$ compared against the non-dimensionalized strain magnitude. 


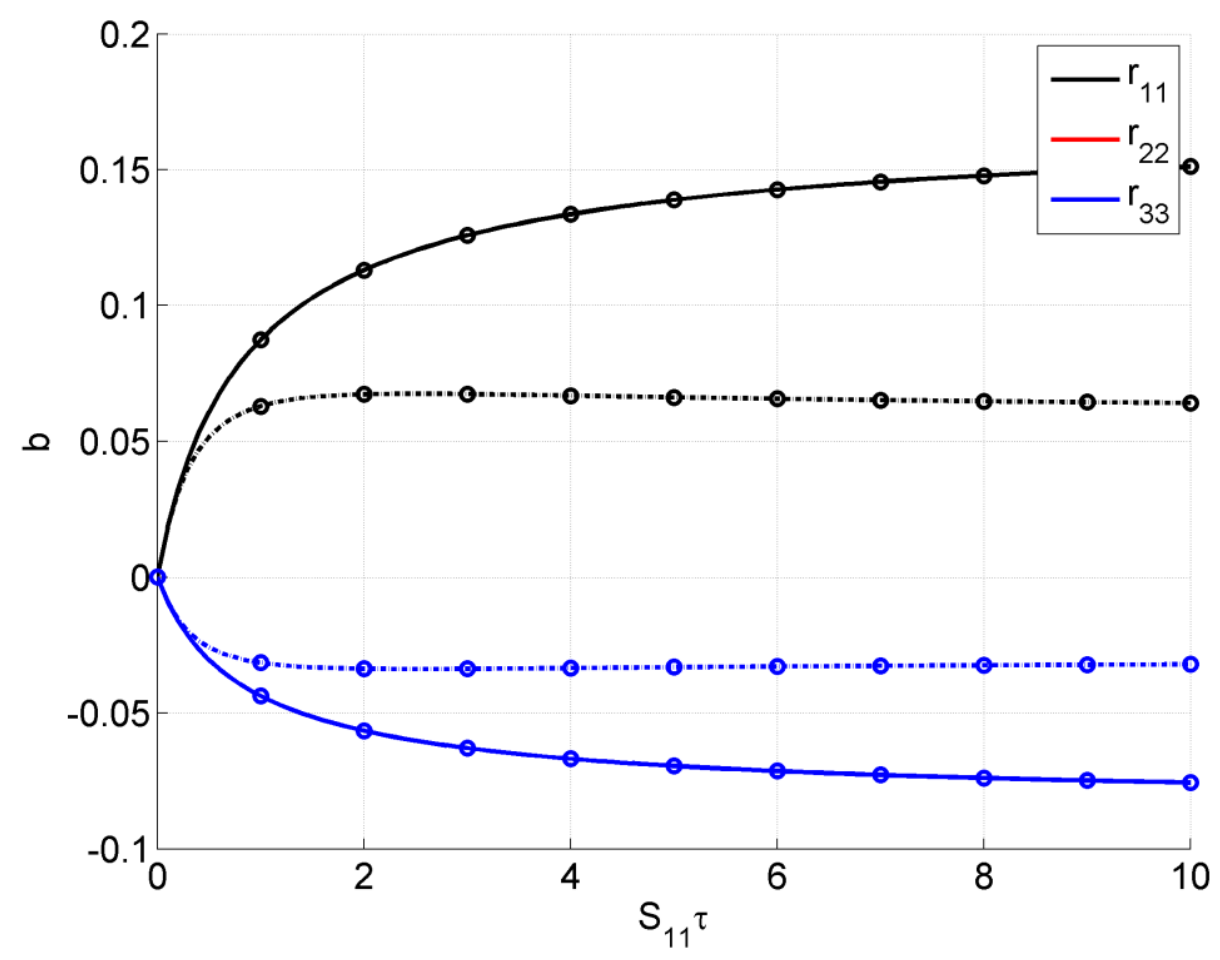

Figure 12. Wall bounded ASBM results under AXE deformation with no frame rotation $\boldsymbol{\Omega}^{f}=\mathbf{0}$ (solid line), and $\Omega^{f}=2 \Gamma$ (dashed-dot line) compared with Langer's results (o).

\section{Plain Strain $(P S)$}

The mean deformation for plain strain is given by

$$
\begin{gathered}
S_{i j}=\left(\begin{array}{ccc}
1 & 0 & 0 \\
0 & -1 & 0 \\
0 & 0 & 0
\end{array}\right), \Omega_{i j}=\left(\begin{array}{ccc}
0 & 0 & 0 \\
0 & 0 & 0 \\
0 & 0 & 0
\end{array}\right), \Omega_{i j}^{f}=\left(\begin{array}{ccc}
0 & 0 & 0 \\
0 & 0 & \Omega^{f} \\
0 & -\Omega^{f} & 0
\end{array}\right) \\
S=\sqrt{2}, \Gamma=1
\end{gathered}
$$

In the Haire model, the value of the slow constant is set to 2. Different levels of frame rotation are introduced as well where $\Omega^{f}=S$ and $\Omega^{f}=2 S$. Figure 13 below displays the comparison of the current implementation and Haire's results. Figure 13 also shows the comparison including frame rotation. The difference in results is negligible once again. 


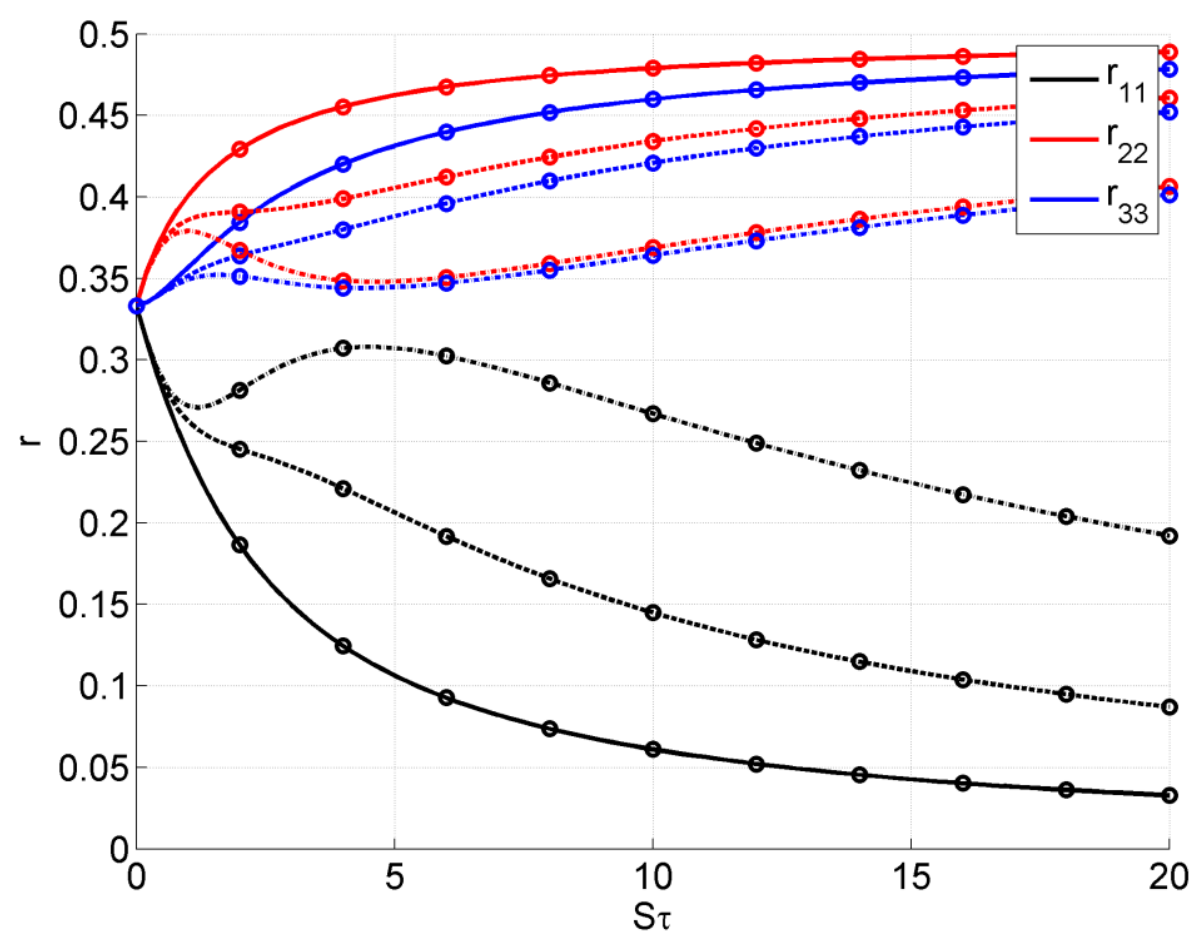

Figure 13. Free shear flow ASBM results under PS deformation with no frame rotation $\Omega^{f}=0$ (solid line), $\Omega^{f}=S$ (dashed line), $\Omega^{f}=2 S$ (dashed-dot line) compared with Haire's results (o).

The Langer implementation does not evaluate this homogeneous flow.

\section{Shear Flow (SH) in a Spanwise Rotating Frame}

The mean deformation for shear flow in a spanwise rotating frame is given by

$$
\begin{gathered}
S_{i j}=\left(\begin{array}{lll}
0 & 1 & 0 \\
1 & 0 & 0 \\
0 & 0 & 0
\end{array}\right), \Omega_{i j}=\left(\begin{array}{ccc}
0 & 1 & 0 \\
-1 & 0 & 0 \\
0 & 0 & 0
\end{array}\right), \Omega_{i j}^{f}=\left(\begin{array}{ccc}
0 & -\Omega^{f} & 0 \\
\Omega^{f} & 0 & 0 \\
0 & 0 & 0
\end{array}\right) \\
S=\frac{1}{\sqrt{2}}, \Gamma=1
\end{gathered}
$$

In the Haire model, the value of the slow constant is varied set to 2 . Different levels of frame rotation are introduced as well varying from a small rotation rate of $\Omega_{3}^{f} / S=0.1$, to a larger rotation rate of $\Omega_{3}^{f} / S=0.5$, and an even larger still rotation rate of $\Omega_{3}^{f} / S=1.0$. Figure 14 below displays the comparison of the current implementation and Haire's 
results. Figure 14 also shows the comparison including frame rotation. The difference in results is negligible.
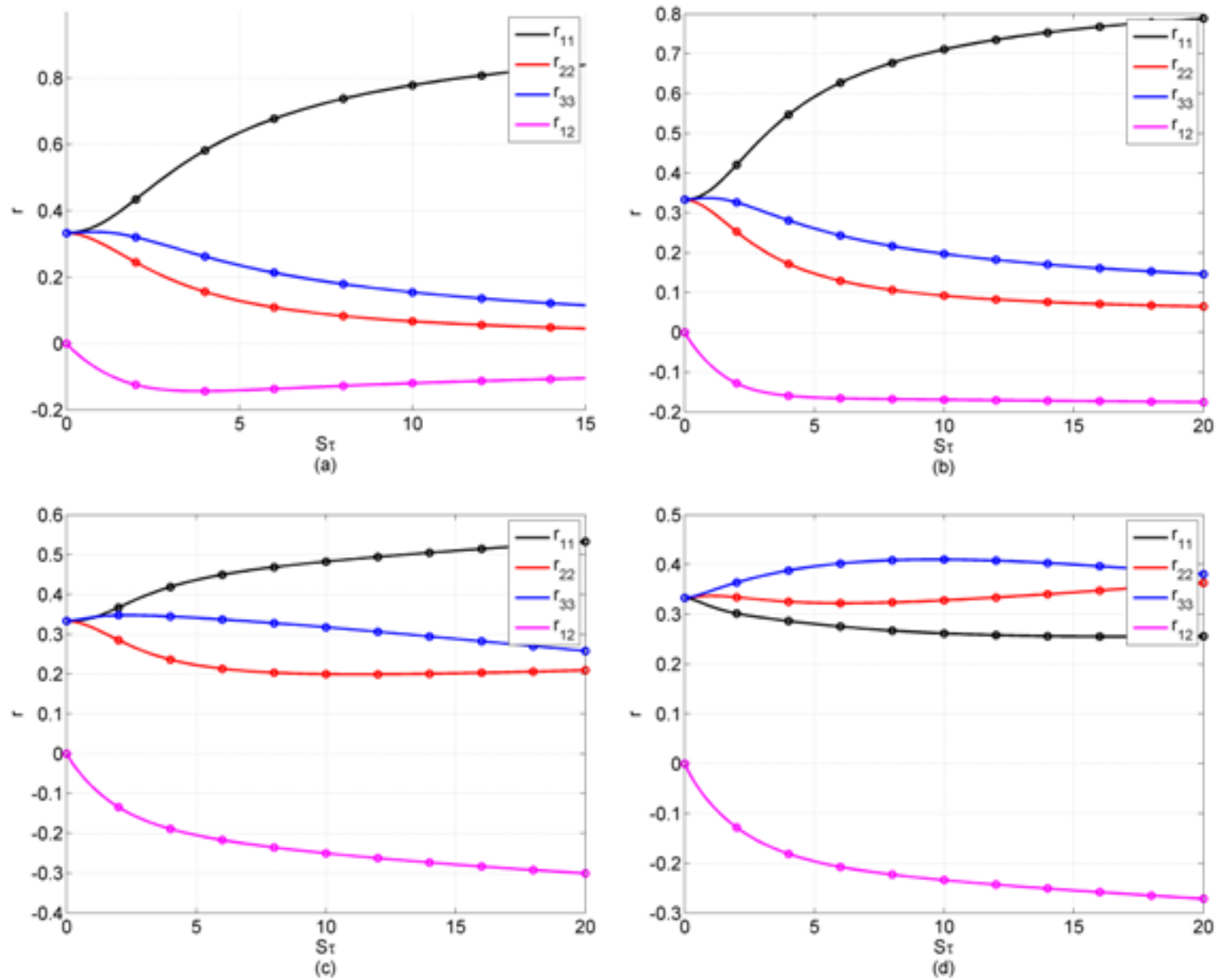

Figure 14. Free shear flow ASBM results under SH with spanwise rotation deformation with no frame rotation $\Omega^{f}=0(\mathrm{a}), \Omega_{3}^{f} / S=0.1(\mathrm{~b}), \Omega_{3}^{f} / S=0.5(\mathrm{c})$, and $\Omega_{3}^{f} / S=1.0(\mathrm{~d})$ compared with Haire's results $(0)$.

The Langer implementation compares the value with the slow constant kept at 1.6. Various frame rotation magnitudes were examined since specific effort was placed on implementation of this flow type. Values of $\eta_{f}=-\Omega_{12}^{f} / \Omega_{12}$ are varied from 0 to 2 by 0.5 . Also counter rotation, $\eta_{f}=-2$, is examined. Figure 15 displays $b_{i j}=r_{i j}-\delta_{i j} / 3$ compared against the non-dimensionalized strain magnitude. 

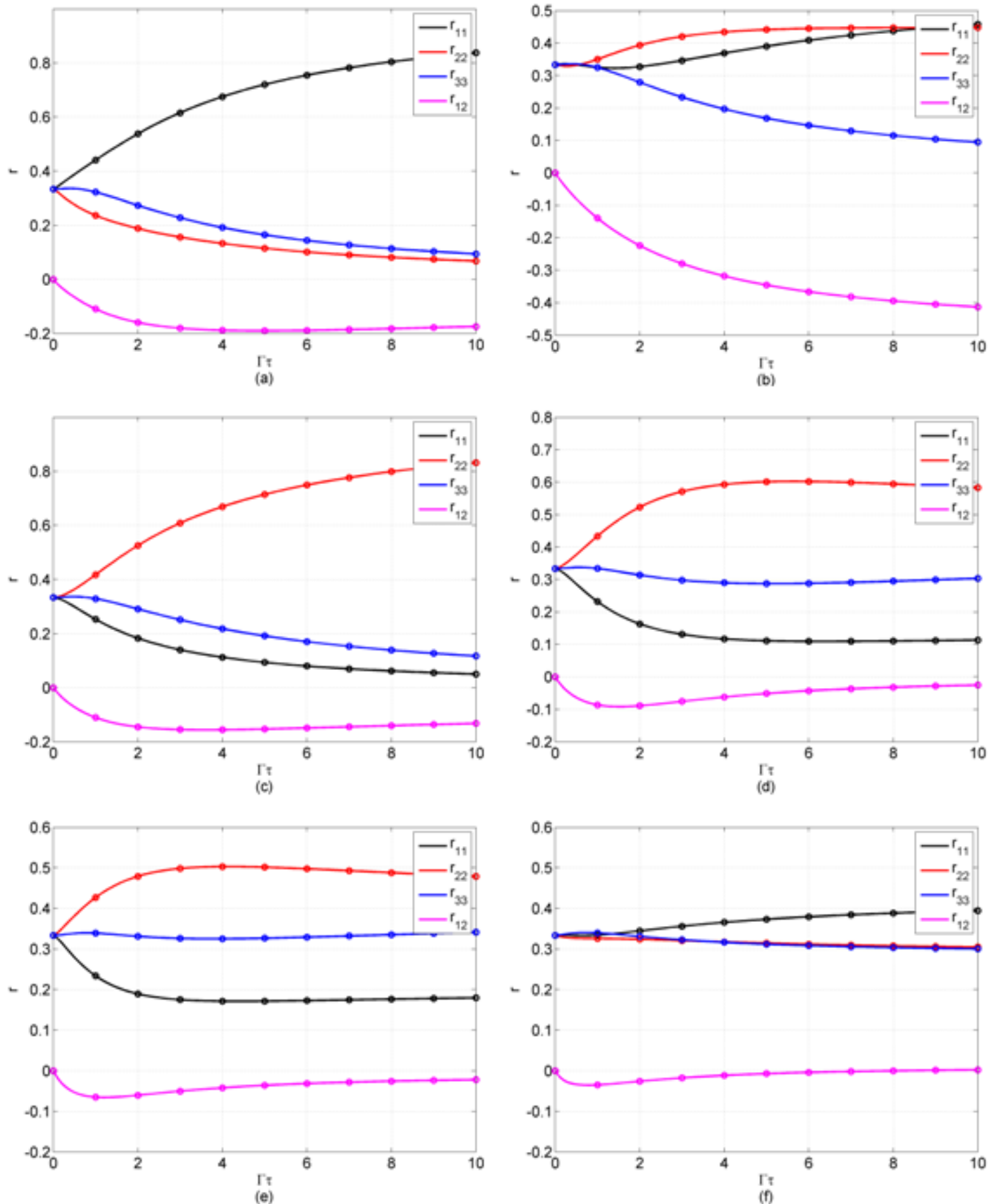

Figure 15. Wall bounded ASBM results under SH with spanwise rotation deformation with no frame rotation $\eta_{f}=0(a), \eta_{f}=0.5(b), \eta_{f}=1.0(c), \eta_{f}=1.5(d), \eta_{f}=2.0(e)$, and $\eta_{f}=-2.0$ (f) compared with Langer's results (o).

\section{Shear Flow (SH) in a Streamwise Rotating Frame}

The mean deformation for shear is given by 


$$
\begin{gathered}
S_{i j}=\left(\begin{array}{lll}
0 & 1 & 0 \\
1 & 0 & 0 \\
0 & 0 & 0
\end{array}\right), \Omega_{i j}= \\
S=\frac{1}{\sqrt{2}}, \Gamma=1
\end{gathered}
$$

In the Haire model, this homogeneous flow was not examined. In the Langer model, however, this homogeneous flow is evaluated to demonstrate its difficulties. Unfortunately, modeling of this flow type does not predict well. This is due to the model being unable to distinguish between the direction of frame rotation. Thus all rotation is seen as stabilizing while in this case it should be destabilizing. Never the less, the model solutions are compared with and without frame rotation of $\Omega_{23}^{f} / \Omega_{12}=-0.5$ below in Figure 16. Again the results are indistinguishable.

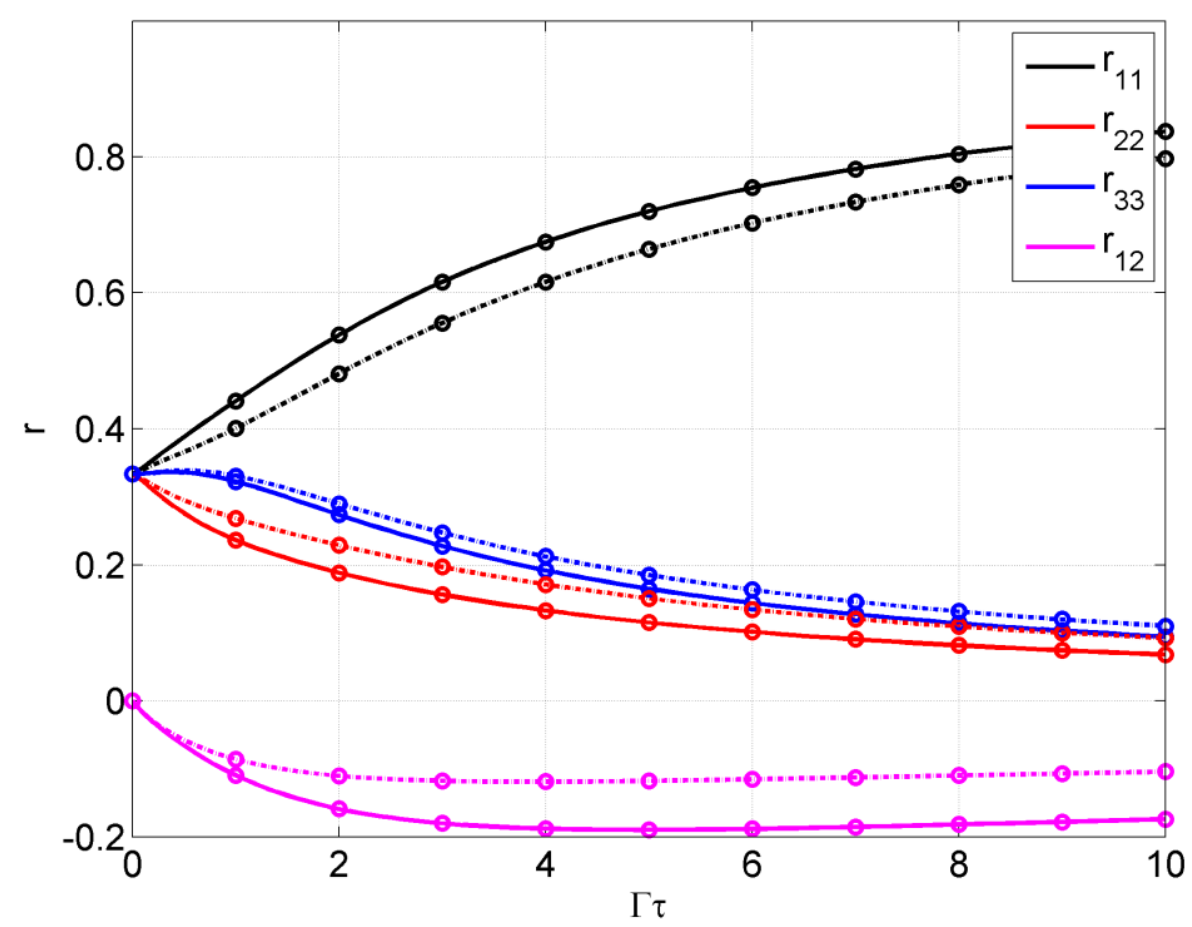

Figure 16. Wall bounded ASBM results under SH with streamwise rotation deformation with no frame rotation $\Omega_{23}^{f} / \Omega_{12}=0$ (solid line), and $\Omega_{23}^{f} / \Omega_{12}=-\mathbf{0 . 5}$ (dashed-dot line) compared with Langer's results (o). 


\section{Realizability}

Cases of axisymmetric contraction, axisymmetric expansion, plain strain, and shear have also been examined on the Anisotropy Invariant (AI) map to ensure that the flows are within physical limits of turbulence. All realistic turbulence must lie within this map. The boundaries are characterized by two states of turbulence. The left line is defined by homogeneous isotropic axisymmetric contraction and the right branch is reached by homogeneous isotropic axisymmetric expansion. The upper limit defines two-component turbulence which is generally reached in the near wall region where the wall-normal component vanishes much faster than others. The three connecting limiting points of the region are also important. The $(0,0)$ point defines isotropic turbulence while the right limit defines the one-component limit. Lastly, the limit of the left branch defines a twocomponent isotropic state. The four homogeneous flow cases are shown below in Figure 17 plotted on the AI map. All flows are realizable. 

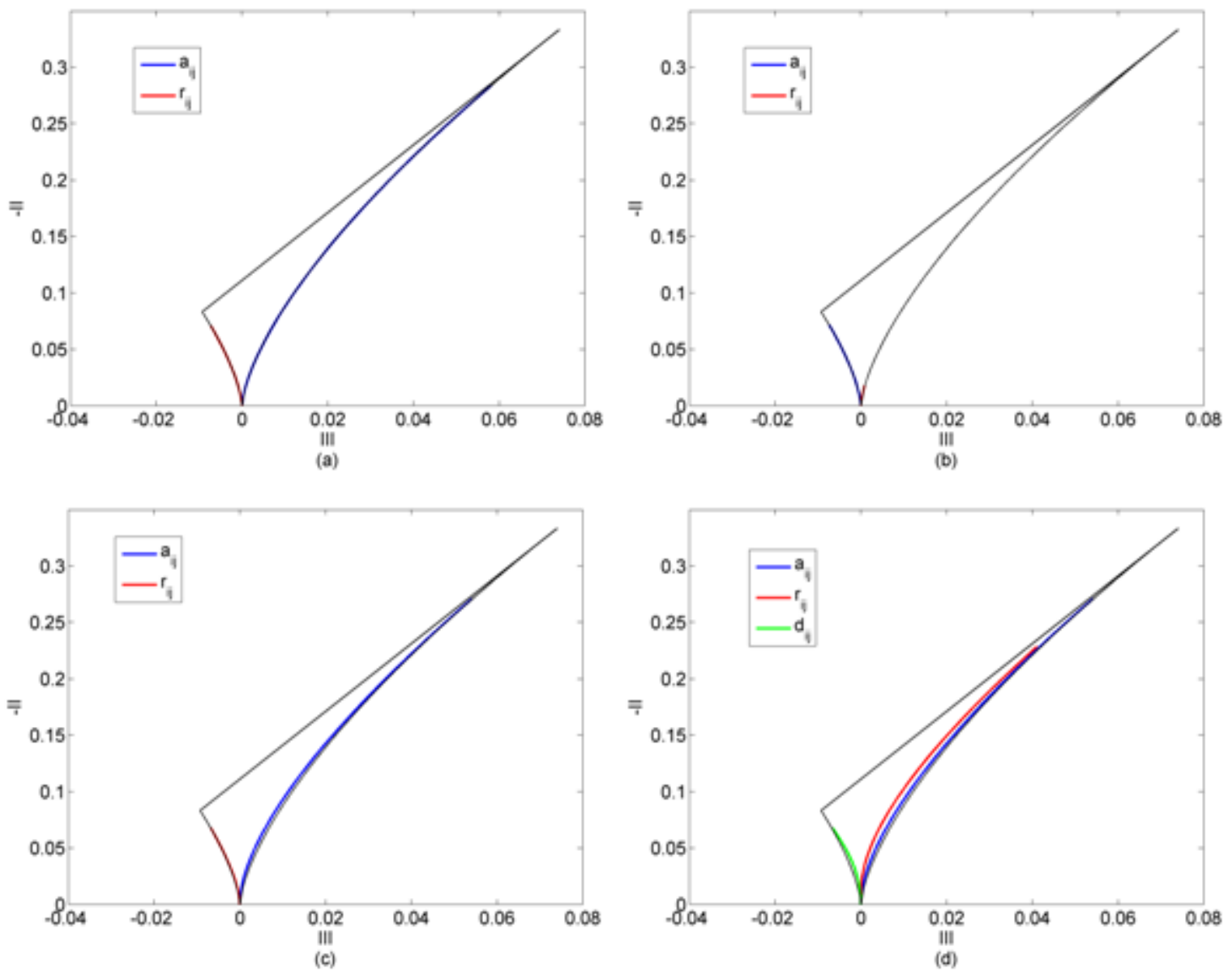

Figure 17. Anisotropy Invariant map for AXC (a), AXE (b), PS (c), and SH (d).

\section{F. ASBM Turbulence Model Coupling}

With the wall bounded ASBM fully defined for a given mean deformation and turbulent time scale, the model can be coupled with various scalar transport equations. Coupling the ASBM with scalar transport equations requires that the scalar transport provide a field distribution of the turbulent kinetic energy along with a means to calculate a turbulent time scale. This required information is available using any of the turbulence model standards, $k-\varepsilon, k-\omega$, or $v^{2}-f$. The model, however, was originally intended to be coupled with scalar transport equations which include both the turbulent kinetic energy, $k$, and the turbulent dissipation rate, $\varepsilon$. This makes coupling schemes with the $k-\varepsilon$ and $v^{2}-f$ 
models a primary choice. Given a field distribution of the mean deformation and the turbulent time scale, the ASBM can be used to determine the Reynolds stresses throughout the field. These Reynolds stresses are used to couple the transport equations and the ASBM. Coupling schemes for each of the models will be denoted as $k-\varepsilon$-ASBM, and $v^{2}-f-A S B M$.

\section{1. $k-\varepsilon-A S B M$}

The $k-\varepsilon$-ASBM couples the ASBM with the standard $k-\varepsilon$ model developed by Launder and Sharma. The standard $k-\varepsilon$ model has been modified slightly in order to include the calculation of Reynolds stresses from the ASBM and thus the anisotropy of turbulence. Besides this modification, the model remains the same. The model equations are displayed below.

$$
\begin{gathered}
\frac{\partial(\rho k)}{\partial t}+\frac{\partial\left(\rho k u_{i}\right)}{\partial x_{i}}=P_{k}+G_{b}-\rho \varepsilon-Y_{M}+\frac{\partial}{\partial x_{j}}\left[\left(\mu+\frac{\mu_{t}}{\sigma_{k}}\right) \frac{\partial k}{\partial x_{j}}\right] \\
\frac{\partial(\rho \varepsilon)}{\partial t}+\frac{\partial\left(\rho \varepsilon u_{i}\right)}{\partial x_{i}}=C_{1 \varepsilon} \frac{\varepsilon}{k}\left(P_{k}+C_{3 \varepsilon} G_{b}\right)-C_{2 \varepsilon} \rho \frac{\varepsilon^{2}}{k}+\frac{\partial}{\partial x_{j}}\left[\left(\mu+\frac{\mu_{t}}{\sigma_{k}}\right) \frac{\partial \varepsilon}{\partial x_{j}}\right]
\end{gathered}
$$

where $P_{k}$ is the generation of turbulence kinetic energy due to mean velocity gradients, $G_{b}$ is the generation of turbulence kinetic energy due to buoyancy, and $Y_{M}$ accounts decreasing spreading rates of turbulence kinetic energy in compressible flows. $P_{k}, G_{b}$ for an ideal gas, and $Y_{M}$ are defined below. The effect of buoyancy has been neglected throughout this analysis however.

$$
\begin{gathered}
P_{k}=-\rho \overline{u_{i}^{\prime} u_{j}^{\prime}} \frac{\partial u_{j}}{\partial x_{i}} \equiv-\rho R_{i j} \frac{\partial u_{j}}{\partial x_{i}} \\
G_{b}=-g_{i} \frac{\mu_{t}}{\rho \operatorname{Pr}_{t}} \frac{\partial \rho}{\partial x_{i}}
\end{gathered}
$$




$$
Y_{M}=2 \rho \varepsilon \frac{k}{a^{2}}
$$

The turbulent viscosity is also calculated based on the original formulation of the $k-\varepsilon$ model.

$$
\mu_{t}=\rho C_{\mu} \frac{k^{2}}{\varepsilon}
$$

The model constants have been calibrated based on experiments by Launder and Sharma. Their values are displayed below.

$$
C_{1 \varepsilon}=1.44 \quad C_{2 \varepsilon}=1.92 \quad C_{3 \varepsilon}=1.92 \quad C_{\mu}=0.09 \quad \sigma_{k}=1.0 \quad \sigma_{\varepsilon}=1.3
$$

2. $v^{2}-f-A S B M$

The $v^{2}$-f-ASBM couples the ASBM with the standard $v^{2}-f$ model developed by Durbin. Since the $v^{2}-f$ model does not require wall functions and already provides damping, this coupling is very synergistic in concept. The velocity scale $v^{2}$, a measure of velocity fluctuation normal to streamlines, is used to damp turbulence transport near inhomogeneities while the elliptic relaxation function models non-local effects. This provides an accurate base for the ASBM to function from in which it can include anisotropic affects of turbulence. The $v^{2}-f$ model has been modified slightly in order to include the calculation of Reynolds stresses from the ASBM and thus the anisotropy of turbulence. Besides this modification, the model remains the same. The model equations are displayed below.

$$
\begin{gathered}
\frac{\partial(\rho k)}{\partial t}+\frac{\partial\left(\rho k u_{i}\right)}{\partial x_{i}}=P_{k}-\rho \varepsilon+\frac{\partial}{\partial x_{j}}\left[\left(\mu+\frac{\mu_{t}}{\sigma_{k}}\right) \frac{\partial k}{\partial x_{j}}\right] \\
\frac{\partial(\rho \varepsilon)}{\partial t}+\frac{\partial\left(\rho \varepsilon u_{i}\right)}{\partial x_{i}}=\frac{C_{1 \varepsilon} P_{k}-C_{2 \varepsilon} \rho \varepsilon}{T}+\frac{\partial}{\partial x_{j}}\left[\left(\mu+\frac{\mu_{t}}{\sigma_{\varepsilon}}\right) \frac{\partial \varepsilon}{\partial x_{j}}\right]
\end{gathered}
$$




$$
\begin{gathered}
\frac{\partial\left(\rho \overline{v^{2}}\right)}{\partial t}+\frac{\partial\left(\rho \overline{v^{2}} u_{i}\right)}{\partial x_{i}}=\rho k f-6 \rho \overline{v^{2}} \frac{\varepsilon}{k}+\frac{\partial}{\partial x_{j}}\left[\left(\mu+\frac{\mu_{t}}{\sigma_{k}}\right) \frac{\partial \overline{v^{2}}}{\partial x_{j}}\right] \\
L^{2} \frac{\partial^{2} f}{\partial x_{k} \partial x_{k}}-f=\frac{1}{T}\left(C_{1}-1\right)\left(\frac{\overline{v^{2}}}{k}-\frac{2}{3}\right)-C_{2} \frac{P_{k}}{\rho k}
\end{gathered}
$$

where $P_{k}$ is again the production of turbulence kinetic energy due to the mean flow velocity gradients as expressed in Eq. 194.

$$
P_{k}=-\rho \overline{u_{i}^{\prime} u_{j}^{\prime}} \frac{\partial u_{j}}{\partial x_{i}} \equiv-\rho R_{i j} \frac{\partial u_{j}}{\partial x_{i}}
$$

$T$ is a measure of the turbulence time scale. The turbulent time scale cannot become less than the Kolmogorov scale and thus $T$ is defined as follows,

$$
T=\max \left[\frac{k}{\varepsilon}, C_{T} \sqrt{\frac{\mu}{\rho \varepsilon}}\right]
$$

where the first term is a measure of the turbulent time scale and the second term is a measure of the Kolmogorov scale. The turbulence length scale is defined similarly again requiring that the scale is larger than that of the Kolmogorov scale. It is defined as follows.

$$
L=C_{L} \max \left[\frac{k^{3 / 2}}{\varepsilon}, C_{\eta}\left(\frac{\mu^{3}}{\rho^{3} \varepsilon}\right)^{1 / 4}\right]
$$

The turbulence viscosity is defined by the velocity scale $v^{2}$ and the turbulent time scale $T$.

$$
\mu_{t}=\rho C_{\eta} \overline{v^{2}} T
$$

Finally, the model constants have been calibrated for coupling with the ASBM. The result of this calibration is shown below. 


$$
\begin{gathered}
C_{\mu}=0.2 \quad \sigma_{k}=1 \quad C_{1}=1.6 \quad C_{2}=0.3 \\
C_{T}=6 \quad C_{L}=0.23 \quad C_{\eta}=60
\end{gathered}
$$




\section{Model Implementation}

The turbulence models discussed in the previous sections, $\zeta-f, k-\varepsilon-\mathrm{ASBM}$, and $v^{2}-f$ ASBM, have been implemented in FLUENT 12 utilizing the User-Defined Function (UDF) capabilities. The following sections detail the UDF theory and the models' implementation.

\section{A. User-Defined Fuctions, Scalars, and Memory}

General CFD solvers such as FLUENT are meant to handle a wide range of solution strategies as well as complex problems. The software, however, cannot accommodate all needs of users. To further the functionality of the software, FLUENT has built in a method for the user to perform custom calculations during preprocessing, post processing, and even during iteration. The custom calculations are called User-Defined Functions (UDFs). UDFs are calculation routines, programmed in $\mathrm{C}$, which can either be hooked to the flow solver or executed independently using calculated variables from the solver or user input. The UDFs act as any $\mathrm{C}$ code implementation and can of course use any standard $\mathrm{C}$ function if the proper header file is included. In addition to standard $\mathrm{C}$ code functionality, FLUENT has a multitude of built in $\mathrm{C}$ functions within the UDF header file which access flow variables and also allow for their modification. It is important to note, however, that not all variables can be modified. These built in functions, called FLUENT macros, allow customized boundary conditions, access to material properties and flow variables throughout the domain, modification of reaction rates, and more. For implementation of turbulence models, customization of boundary 
conditions, adjustment of computed values every iteration, modification of turbulence viscosity, and use of both User-Defined Scalars (UDS) and User-Defined Memory (UDM) are necessary.

User-Defined Scalars are FLUENT's method of solving generic transport equations utilizing the solver's capabilities. The generic transport equation is of the form

$$
\frac{\partial\left(\rho \phi_{k}\right)}{\partial t}+\frac{\partial}{\partial x_{i}}\left(\begin{array}{cc}
F_{i} \phi_{k} & -\underbrace{\Gamma_{k} \frac{\partial \phi_{k}}{\partial x_{i}}}_{\text {convection }}
\end{array}\right)=\underbrace{S_{\phi_{k}}}_{\text {diffusion }} \text { source }
$$

where $\phi_{k}$ is the UDS, $k$ is the UDS index, $F_{i}$ is a user-defined flux function, $\Gamma_{k}$ is a userdefined diffusivity, and $S_{\phi_{k}}$ is a user-defined source term. Each of the convection, diffusion, and source terms can be customized for a specific transport equation. In addition, these terms can be modified for existing transport equations such the turbulent kinetic energy while using the built in $k$ - $\varepsilon$ model. Since FLUENT calculates the gradient of a UDS automatically, it can also be useful to store calculated values as a UDS without solving the transport equation. Calculated values can also be stored as a User-Defined Memory (UDM) which simply allows the variable to be accessed in different FLUENT macros.

\section{B. User-Defined Function Architecture}

FLUENT UDFs can be implemented in two ways. The first method, denoted an interpreted UDF, is to interpret the UDF source file at runtime. The source code is compiled in an intermediate, architecture-independent machine code using a C preprocessor. The disadvantage of this method is a penalty in performance due to the runtime compiling and also the fact that only a limited subset of FLUENT macros is 
available. It does, however, allow the UDF to be shared seamlessly with different FLUENT versions and even operating systems. The second method, denoted a compiled UDF, is compiled using the same method as the FLUENT executable itself. This method allows for more complex UDFs to be implemented and also has no performance penalty. All turbulence model implementations have been read as compiled UDFs.

The use of UDFs with the FLUENT solver has limited ability to modify the solver method. Each of the macros involved with adding functionality to the solver is executed at a specific time in the iteration process. The solver's architecture including UDF macro functions for each iteration is displayed below in Figure 18.

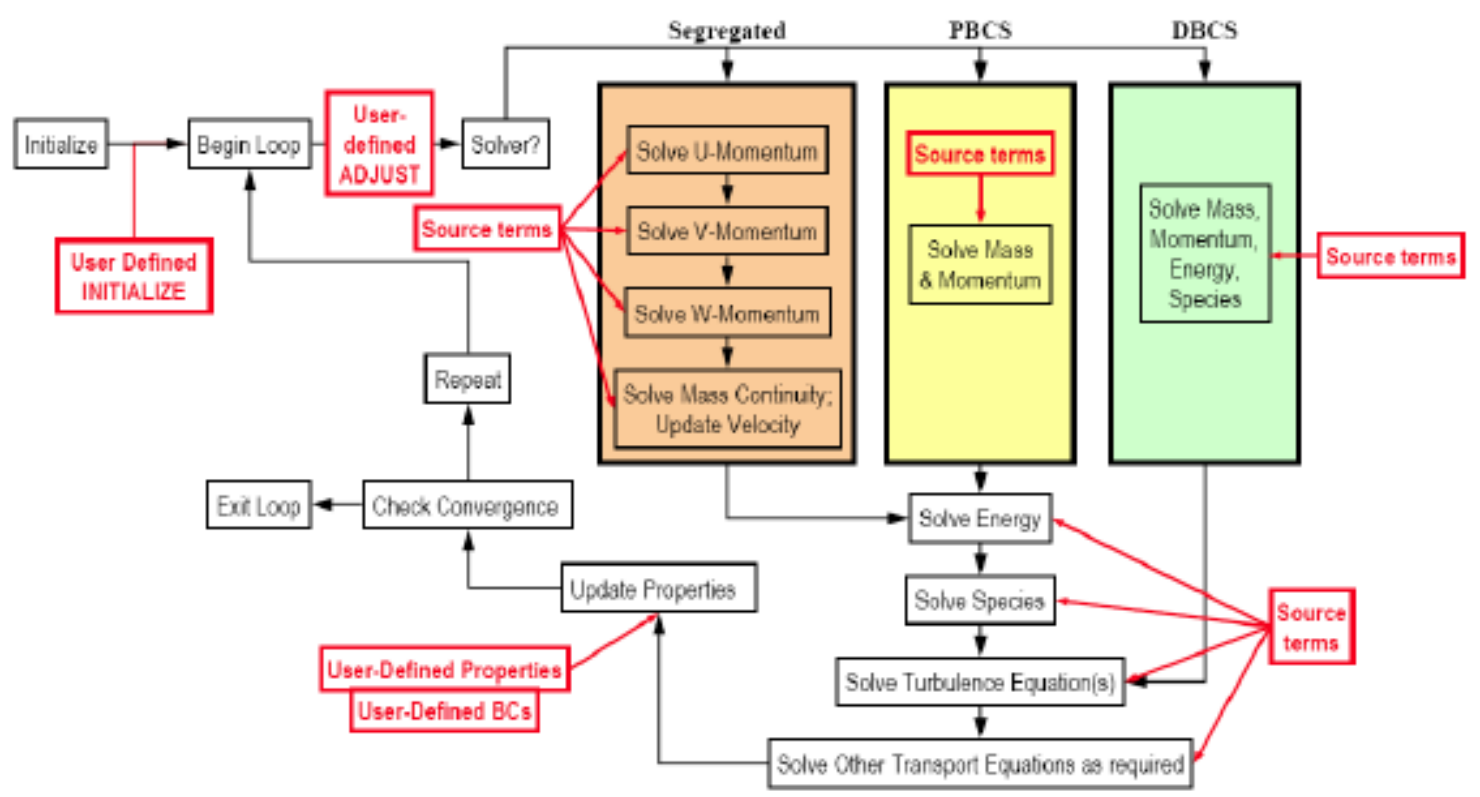

Figure 18. Architecture for user access to the FLUENT solver.

Applying a compiled UDF to FLUENT requires six general steps. These general steps will, of course, vary greatly for each implementation.

1. Create the UDF source code

2. Create a FLUENT case

3. Compile the UDF 


\section{Load the UDF}

\section{Attach UDF to the FLUENT solver \\ 6. Solve}

\section{Implementation of the $\zeta-f$ model}

The $\zeta-f$ turbulence model was written for use in FLUENT via user-defined functions. The transport equations for each of the four variables were written in the format of Eq. 207 and solved with the FLUENT solver. The turbulent kinetic energy $k$, turbulent dissipation rate $\varepsilon$, the normalized velocity scale $\zeta$, and the elliptic relaxation factor $f$ were transported according to this equation. The defining flux, diffusion, and source terms are displayed below in Table 3 for each of the transport variables.

Table 3. User-Defined Function terms for the $\zeta-f$ turbulence model.

\begin{tabular}{|c|c|c|c|c|}
\hline $\begin{array}{c}\text { Transported } \\
\text { Variable, } \phi_{k}\end{array}$ & $\begin{array}{c}\text { Index, } \\
k\end{array}$ & $\begin{array}{c}\text { Flux } \\
\text { Function, } \\
F_{i}\end{array}$ & $\begin{array}{c}\text { Source, } \\
S_{\phi_{k}}\end{array}$ & $\begin{array}{c}\text { Diffusivity, } \\
\Gamma_{k}\end{array}$ \\
\hline $\begin{array}{c}\text { Turbulent } \\
\text { Kinetic Energy, } \\
k\end{array}$ & 0 & $\dot{m}=\rho u_{i}$ & $P_{k}-\frac{\rho^{2} C_{\mu} \zeta k^{2}}{\mu_{t}}$ & $\mu+\frac{\mu_{t}}{\sigma_{k}}$ \\
\hline $\begin{array}{c}\text { Turbulent } \\
\text { Dissipation Rate, } \\
\varepsilon\end{array}$ & 1 & $\dot{m}=\rho u_{i}$ & $\frac{\left(C_{\varepsilon 1}^{\prime} P_{k}-\rho C_{\varepsilon 2} \varepsilon\right)}{T}$ & $\mu+\frac{\mu_{t}}{\sigma_{\varepsilon}}$ \\
\hline $\begin{array}{c}\text { Normalized } \\
\text { Velocity Scale, } \\
\zeta\end{array}$ & 2 & $\dot{m}=\rho u_{i}$ & $\rho f-\frac{\zeta}{k}\left(P_{k}+\rho \varepsilon\right)$ & $\mu+\frac{\mu_{t}}{\sigma_{\zeta}}$ \\
\hline $\begin{array}{c}\text { Elliptic } \\
\text { Relaxation } \\
\text { Factor, } \\
f\end{array}$ & 3 & 0 & $\frac{1}{L^{2}}\left\{\frac{1}{T}\left[\left(C_{1}-1+C_{2} \frac{P_{k}}{\rho \varepsilon}\right)\left(\zeta-\frac{2}{3}\right)\right.\right.$ & -1 \\
\hline
\end{tabular}

For the turbulent kinetic energy source term, the second term is modified from the original transport equation in order for the source to be a function of the transport variable. This is done in order to improve stability of the scheme. When the source is a 
function of the transport variable, FLUENT is able to treat the source implicitly as opposed to explicitly which helps make the model more robust.

Examining the source and diffusivity term for the elliptic relaxation factor as compared to the transport equation defined earlier, the length scale has been divided through onto the source. While the length scale squared is a diffusive term, it is outside of the Laplacian and must be treated accordingly as compared to the general transport equation that FLUENT solves. This also requires that the diffusivity be set to negative one to mimic the general transport equation. Since both the turbulent length scale and time scale are in the denominator of the source term, it is especially important to ensure they do not become zero and create a singularity. The limits of the Kolmogorov scales proposed earlier ensure this.

The implementation is complete by modifying the turbulent viscosity used within FLUENT. This couples the $\zeta-f$ turbulence model with the mean flow in the frame work of an eddy-viscosity turbulence model.

Lastly, the initial conditions for the transport variables can have a large effect on the stability. For the turbulent kinetic energy and the turbulent dissipation rate, a well established method is to specify the turbulent intensity and the viscosity ratio and back out both $k$ and $\varepsilon$. Due to the zero wall boundary condition for the elliptic relaxation factor, it is also reasonable to initialize $f$ as zero throughout the domain. For the normalized velocity scale, the equilibrium value of $2 / 3$ is reasonable. The initial conditions as well as the wall boundary conditions are shown below in

Table 4. 
Table 4. $\zeta-f$ initial and wall boundary conditions.

\begin{tabular}{|c|c|c|c|c|}
\cline { 2 - 5 } \multicolumn{1}{c|}{} & $k$ & $\varepsilon$ & $\zeta$ & $f$ \\
\hline $\begin{array}{c}\text { Initial } \\
\text { Conditions }\end{array}$ & $\frac{3}{2}(I U)^{2}$ & $\frac{\rho C_{\mu} k^{2}}{\mu_{\text {ratio }} \mu}$ & $2 / 3$ & 0 \\
\hline $\begin{array}{c}\text { Wall Boundary } \\
\text { Conditions }\end{array}$ & 0 & $\frac{2 v k_{p}}{y_{p}^{2}}$ & 0 & 0 \\
\hline
\end{tabular}

Here $U$ is the velocity magnitude, $I$ is the turbulent intensity, and $\mu_{\text {ratio }}$ is the viscosity ratio. $C_{\mu}$ is the $k-\varepsilon$ model value of 0.09 . The model constants for the $\zeta-f$ turbulence model are summarized again below in Table 5 .

Table 5. Calibrated $\zeta-f$ model constants.

\begin{tabular}{|c|c|c|c|c|c|c|c|c|c|c|c|c|}
\hline$\mu_{t}$ & $k$ & \multicolumn{4}{|c|}{$\varepsilon$} & $\zeta$ & \multicolumn{2}{|c|}{$f$} & \multicolumn{2}{|c|}{$T$} & \\
\hline$\overline{C_{\mu}}$ & $\sigma_{k}$ & $\overline{C_{\varepsilon 1}^{\prime}}$ & $C_{\varepsilon 1}$ & $C_{\varepsilon 2}$ & $\overline{\sigma_{\varepsilon}}$ & $\overline{\sigma_{\zeta}}$ & $\overline{C_{1}}$ & $\overline{C_{2}}$ & $\overline{C_{T}}$ & $C_{\tau}$ & $\overline{C_{L}}$ & $\overline{C_{\eta}}$ \\
\hline 0.22 & 1.0 & $C_{\varepsilon I}(1+0.012) / \zeta$ & 1.4 & 1.9 & 1.3 & 1.2 & 1.4 & 0.65 & 0.6 & 6.0 & 0.28 & 65 \\
\hline
\end{tabular}

\section{Implementation of the $k$ - $\varepsilon$-ASBM}

The implementation of the $k$ - $\varepsilon$-ASBM varies from the implementation of the $\zeta-f$ model. The $k-\varepsilon$-ASBM is built onto the existing standard $k-\varepsilon$ model within FLUENT. This means that no additional transport equations are required to be solved. The existing transport equation source terms are modified, however. The ASBM Reynolds stress also needs to be calculated within an adjust function. Table 6 below displays the adjusted source terms for the standard $k-\varepsilon$ model. These source terms and the adjust function are implemented using a UDF. 


\section{Table 6. $k$ - $\varepsilon$-ASBM Source Terms}

\begin{tabular}{|c|c|}
\hline Transported Variable, $\phi_{k}$ & Source, \\
$S_{\phi_{k}}$
\end{tabular}

The adjust function calculates the Reynolds stresses in each cell using the ASBM and the Newton-Raphson method described earlier. The Reynolds stresses are then used to calculate the production of turbulent kinetic energy which is replaced in the source term presented above. The blockage parameter is calculated using a UDS. The flux, diffusivity, and source terms are shown below in Table 7.

Table 7. Blockage parameter user-defined scalar definition.

\begin{tabular}{|c|c|c|c|c|}
\hline $\begin{array}{c}\text { Transported } \\
\text { Variable, } \phi_{k}\end{array}$ & $\begin{array}{c}\text { Index, } \\
k\end{array}$ & $\begin{array}{c}\text { Flux } \\
\text { Function, } \\
F_{i}\end{array}$ & $\begin{array}{c}\text { Source, } \\
S_{\phi_{k}}\end{array}$ & $\begin{array}{c}\text { Diffusivity, } \\
\Gamma_{k}\end{array}$ \\
\hline $\begin{array}{c}\text { Blockage } \\
\text { Parameter, } \Phi\end{array}$ & 0 & 0 & $\Phi / L^{2}$ & -1 \\
\hline
\end{tabular}

Just as with the elliptic relaxation factor, the diffusive length scale squared has been moved to the source side of the equation. This makes it especially important to ensure that $L$ does not ever reach zero. This is again achieved by limiting the length scale using the Kolmogorov scale.

The Reynolds stresses calculated using the ASBM are also stored as UDS in order to have access to the gradients throughout the flow field. This is only important when trying to couple the ASBM with the mean flow without using the Boussinesq approximation. In FLUENT, however, it is not possible to modify the mean flow coupling directly since there is no macro for the Boussinesq approximation. As a result, 
the Boussinesq approximation must also be calculated using macros that are available. The resulting approximation of the Boussinesq approximation is stored as a UDS as well in order to have access to the gradients. The mean flow calculation is then adjusted by the addition of source terms. The ASBM Reynolds stress gradients are added while the approximated Boussinesq assumption gradients are subtracted. It is extremely important to calculate both of these quantities in each face as well as each cell to ensure smoother gradients. The source terms are summarized below in Table 8 .

Table 8. Velocity source for mean flow coupling.

\begin{tabular}{|c|c|}
\hline $\begin{array}{c}\text { Transported } \\
\text { Variable } \\
\phi_{k}\end{array}$ & $\begin{array}{c}\text { Source, } \\
S_{u_{i}}\end{array}$ \\
\hline $\begin{array}{c}\text { Velocity } \\
, u_{i}\end{array}$ & $\frac{\partial\left(-\rho \overline{u_{i} u_{j}}\right)}{\partial x_{j}}-\frac{\partial}{\partial x_{j}}\left(\mu_{t}\left(\frac{\partial u_{i}}{\partial x_{j}}+\frac{\partial u_{j}}{\partial x_{i}}\right)-\frac{2}{3}\left(\rho k+\mu_{t} \frac{\partial u_{k}}{\partial x_{k}}\right) \delta_{i j}\right)$ \\
\hline
\end{tabular}

It is also important to note that while FLUENT is limited to modification of the flow in this manner, previous implementations of the ASBM in different flow solvers have utilized a similar method in order to improve stability. Since the ASBM Reynolds stresses cannot be treated implicitly, this method relies on the implicit treatment of the Boussinesq approximation for stability. Unfortunately, the ASBM was not coupled to the mean flow in this study due to the limits of FLUENT's data access macros. Without direct access to the Boussinesq approximation and its gradients, the previously mentioned approximation does not cancel out properly using the source term near developing flow. This results in stability issues and eventually inaccurate prediction of the turbulent variables and thus the flow. 


\section{E. Implementation of the $v^{2}-f$-ASBM}

The $v^{2}-f$-ASBM is implemented similarly to both the $k$ - $\varepsilon$-ASBM and the $\zeta-f$ model. It was written via FLUENT user-defined functions and relies on the transport of five variables, four for the $v^{2}-f$ model and one for the ASBM blockage, as well as the calculation of the ASBM Reynolds stresses. The transport equations for each of the five variables were written in the format of Eq. 207 and solved with the FLUENT solver. The turbulent kinetic energy $k$, turbulent dissipation rate $\varepsilon$, the velocity scale $v^{2}$, the elliptic relaxation factor $f$, and the blockage parameter $\Phi$ were transported according to this equation. The defining flux, diffusion, and source terms are displayed below in Table 9 for each of the transport variables.

Table 9. User-Defined Function terms for the $v^{2}-f$-ASBM turbulence model.

\begin{tabular}{|c|c|c|c|c|}
\hline $\begin{array}{c}\text { Transported } \\
\text { Variable, } \phi_{k}\end{array}$ & $\begin{array}{c}\text { Index, } \\
k\end{array}$ & $\begin{array}{c}\text { Flux } \\
\text { Function, } \\
F_{i}\end{array}$ & $\begin{array}{c}\text { Source, } \\
S_{\phi_{k}}\end{array}$ & $\begin{array}{c}\text { Diffusivity, } \\
\Gamma_{k}\end{array}$ \\
\hline $\begin{array}{c}\text { Turbulent } \\
\text { Kinetic Energy, } \\
k\end{array}$ & 0 & $\dot{m}=\rho u_{i}$ & $-\rho u_{i} u_{j} \frac{\partial u_{i}}{\partial x_{j}}-\frac{\rho^{2} C_{\mu} v^{2} k}{\mu_{t}}$ & $\mu+\frac{\mu_{t}}{\sigma_{k}}$ \\
\hline $\begin{array}{c}\text { Turbulent } \\
\text { Dissipation Rate, } \\
\varepsilon\end{array}$ & 1 & $\dot{m}=\rho u_{i}$ & $\frac{\left(-C_{\varepsilon 1}^{\prime} \rho \overline{u_{i} u_{j}} \frac{\partial u_{i}}{\partial x_{j_{k}}}-\rho C_{\varepsilon 2} \varepsilon\right.}{T}$ & $\mu+\frac{\mu_{t}}{\sigma_{\varepsilon}}$ \\
\hline $\begin{array}{c}\text { Velocity Scale, } \\
v^{2}\end{array}$ & 2 & $\dot{m}=\rho u_{i}$ & $\frac{\rho k f-6 \rho v^{2}}{T}$ & $\mu+\frac{\mu_{t}}{\sigma_{k}}$ \\
\hline $\begin{array}{c}\text { Elliptic } \\
\text { Relaxation } \\
\text { Factor, } \\
f\end{array}$ & 3 & 0 & $-\frac{f}{L^{2}}-\frac{1}{T L^{2}} \frac{\left(C_{1}-6\right) v^{2}}{k}$ & 1 \\
\hline $\begin{array}{c}\text { Blockage } \\
\text { Parameter, } \\
\Phi\end{array}$ & 4 & 0 & $\left.-\frac{2}{3}\left(C_{1}-1\right)\right]+\frac{-C_{2} \rho \overline{u_{i} u_{j}} \frac{\partial u_{i}}{\partial x_{j}}}{\rho k L^{2}}$ & \\
\hline
\end{tabular}


For the turbulent kinetic energy source term, the second term is modified from the original transport equation just as with the $\zeta-f$ model in order for the source to be a function of the transport variable. This is done in order to improve stability of the scheme. The implicit treatment makes the model more robust as well.

Both the elliptic relaxation factor and the blockage parameter require that the length scale never reach zero to avoid a singularity. The time scale has this same requirement for the elliptic relaxation factor. This is again accomplished by limiting the values to the Kolmogorov scales. It is also important to note that the length scale used for the blockage parameter is defined differently than the length scale defined for the elliptic relaxation factor.

The implementation is complete by modifying the turbulent viscosity used within FLUENT. This couples the $v^{2}-f$ turbulence model with the mean flow in the frame work of an eddy-viscosity turbulence model.

Just as with the $\zeta-f$ model the initial conditions for the transport variables can have a large effect on the stability. The initial conditions for the $v^{2}-f$-ASBM can be treated very similarly. For the turbulent kinetic energy and the turbulent dissipation rate, a well established method is to specify the turbulent intensity and the viscosity ratio and back out both $k$ and $\varepsilon$. Due to the zero wall boundary condition for the elliptic relaxation factor, it is also reasonable to initialize $f$ as zero throughout the domain as well. For the velocity scale, the equilibrium value of $2 / 3 k$ can be used with the initial value of $k$. Lastly, the initial condition for the blockage parameter $\Phi$ is set to zero which is the equation's solution value throughout most of the domain. The initial conditions as well as the wall boundary conditions are shown below in Table 10. 
Table 10. $v^{2}-f$ initial and wall boundary conditions.

\begin{tabular}{|c|c|c|c|c|c|}
\cline { 2 - 6 } \multicolumn{1}{c|}{} & $k$ & $\varepsilon$ & $v^{2}$ & $f$ & $\Phi$ \\
\cline { 2 - 5 } & $\frac{3}{2}(I U)^{2}$ & $\frac{\rho C_{\mu} k^{2}}{\mu_{\text {ratio }} \mu}$ & $2 / 3 k$ & 0 & 0 \\
\hline $\begin{array}{c}\text { Initial } \\
\text { Conditions }\end{array}$ & $\frac{2 v k_{p}}{y_{p}^{2}}$ & 0 & 0 & 1 \\
\hline $\begin{array}{c}\text { Wall } \\
\text { Coundary }\end{array}$ & 0 & & & \\
\hline
\end{tabular}

Here $U$ is the velocity magnitude, $I$ is the turbulent intensity, and $\mu_{\text {ratio }}$ is the viscosity ratio. $C_{\mu}$ is the $k-\varepsilon$ model value of 0.09 .

The ASBM portion of the model is generally only utilized after a converged solution for the $v^{2}-f$ model has been reached. This is to improve stability and ensure convergence. Therefore the model implementation includes the original source terms that have not been modified. The model constants, however, are still modified and are again summarized below in Table 11.

Table 11. $v^{2}-f$-ASBM model constants

\begin{tabular}{|c|c|c|c|c|c|c|c|c|c|c|c|c|c|}
\hline $\boldsymbol{\mu}_{\boldsymbol{t}}$ & $\boldsymbol{k}$ & \multicolumn{3}{|c|}{$\boldsymbol{\varepsilon}$} & $\boldsymbol{v}^{2}$ & \multicolumn{2}{c|}{$\boldsymbol{f}$} & \multicolumn{2}{c|}{$\boldsymbol{T}$} & \multicolumn{2}{c|}{$\boldsymbol{L}_{\boldsymbol{v} 2 f}$} & \multicolumn{2}{c|}{$\boldsymbol{L}_{\text {ASBM }}$} \\
\hline$C_{\mu}$ & $\sigma_{k}$ & $C^{\prime}{ }_{\varepsilon 1}$ & $C_{\varepsilon 1}$ & $C_{\varepsilon 2}$ & $\sigma_{\varepsilon}$ & $\sigma_{k}$ & $C_{1}$ & $C_{2}$ & $C_{T}$ & $C_{L}$ & $C_{\eta}$ & $C_{L}$ & $C_{\eta}$ \\
\hline 0.25 & 1.0 & $C_{\varepsilon 1}\left(1+0.05 \sqrt{k / v^{2}}\right)$ & 1.4 & 2.1 & 1.3 & 1.0 & 1.4 & 0.3 & 6.0 & 0.23 & 70 & 0.8 & 50 \\
& & & & & & & & & & & \\
\hline
\end{tabular}




\section{Results}

Three validation cases have been performed for the application of the $\zeta-f$ model and ASBM including the flat plate in turbulent subsonic flow, backward facing step and the S3H4 2D hill. For each of the validation cases, Spalart-Allmaras, $k-\varepsilon, k-\omega, k-\omega-\mathrm{SST}$, and

the $v^{2}-f$ model have been used for comparison. The $v^{2}-f$ model used for comparison was written using FLUENT user-defined functions and validated previously. The implemented $\zeta-f$ model and ASBM were also written using FLUENT user-defined functions. The model constants of each model have been calibrated accordingly. Built in models of Spalart-Allmaras, $k-\varepsilon, k-\omega$, and $k-\omega$-SST were used for their evaluation. The computational data for each validation case is compared against experimental data and theoretical data when available.

\section{A. Flat plate in turbulent, subsonic flow}

The $\zeta-f$ model and $v^{2}-f-A S B M$ along with Spalart-Allmaras, $k-\varepsilon, k-\omega, k-\omega-S S T$, and $k$ - $\omega$ were used to solve turbulent subsonic flow over a flat plate. The Reynolds number at the end of the plate was $1 \mathrm{e} 7$ to allow adequate boundary layer formation at the data station of $\operatorname{Re}_{\mathrm{x}}=4.6 \times 10^{6}$. The skin friction coefficient generated using each of the models was compared with a theoretical relationship for turbulent flat plate flow and experimental data. The theoretical relationship is shown below.

$$
C_{f}=\frac{0.027}{\left(\operatorname{Re}_{x}\right)^{1 / 7}}
$$

Figure 19 below displays the comparison of the skin friction coefficients obtained from each model. Each model used seems to under predict the skin friction slightly with 
the $k-\omega$ models result in the worst correlation compared to the theoretical relationship. The $\zeta-f$ model predicts well especially at the start of the plate. It does, however, deviate further as the Reynolds number increases. This occurs even more prominently if Popovac's model constants are used. While this effect could be avoided completely by modifying model constants in a different manner, this results in worse prediction of boundary layer profile. The $v^{2}-f$-ASBM displays the opposite effect. This is due to the difficulty the model has during boundary layer formation and seems to propagate down stream even after stability issues have been resolved. The model prediction is still adequate especially far from the plate edge.

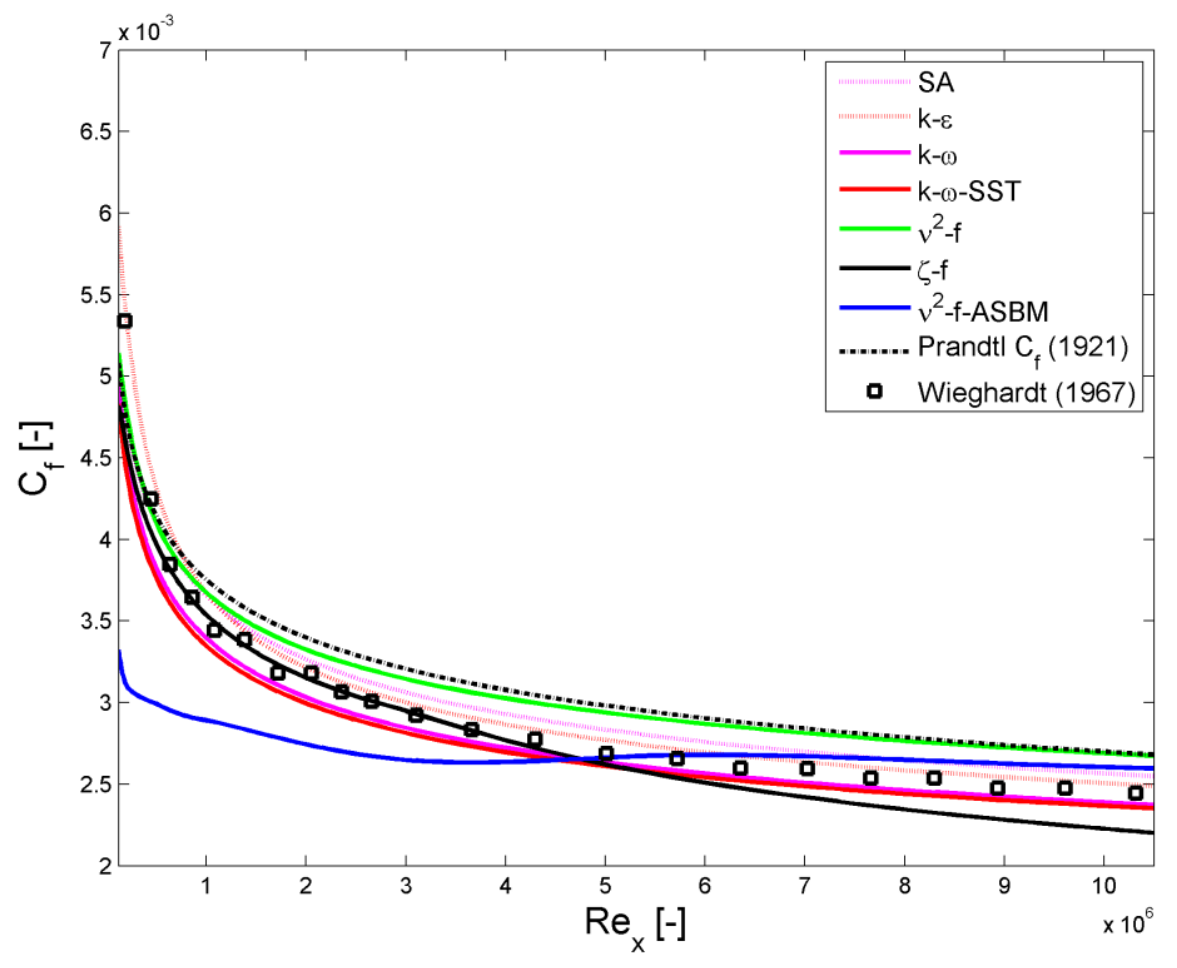

Figure 19. Skin fiction coefficient distribution for a turbulent flat plate in subsonic flow.

Boundary layer velocity profiles for the turbulent flat plate along with experimental data by Wieghardt are also shown below in Figure 20. It is clear that each of the turbulence models captures the general trend of the velocity profile. The $\zeta-f$ model 
predicts the boundary profile very well matching Spaldings law and the Wieghardt data. The $v^{2}-f$-ASBM produces an acceptable profile although it does not match as well as the $\zeta-f$ model. It is important to note that even on such a simple validation case, it was difficult to produce acceptable results. The length scale used, particularly when the Kolmogorov scales begin to dominate, has a very large effect on the log layer constant $B$. The convergence of the model is also very slow and the model results vary even when residuals are no longer dropping. Figure 21 below displays the results of the two implemented models alone.

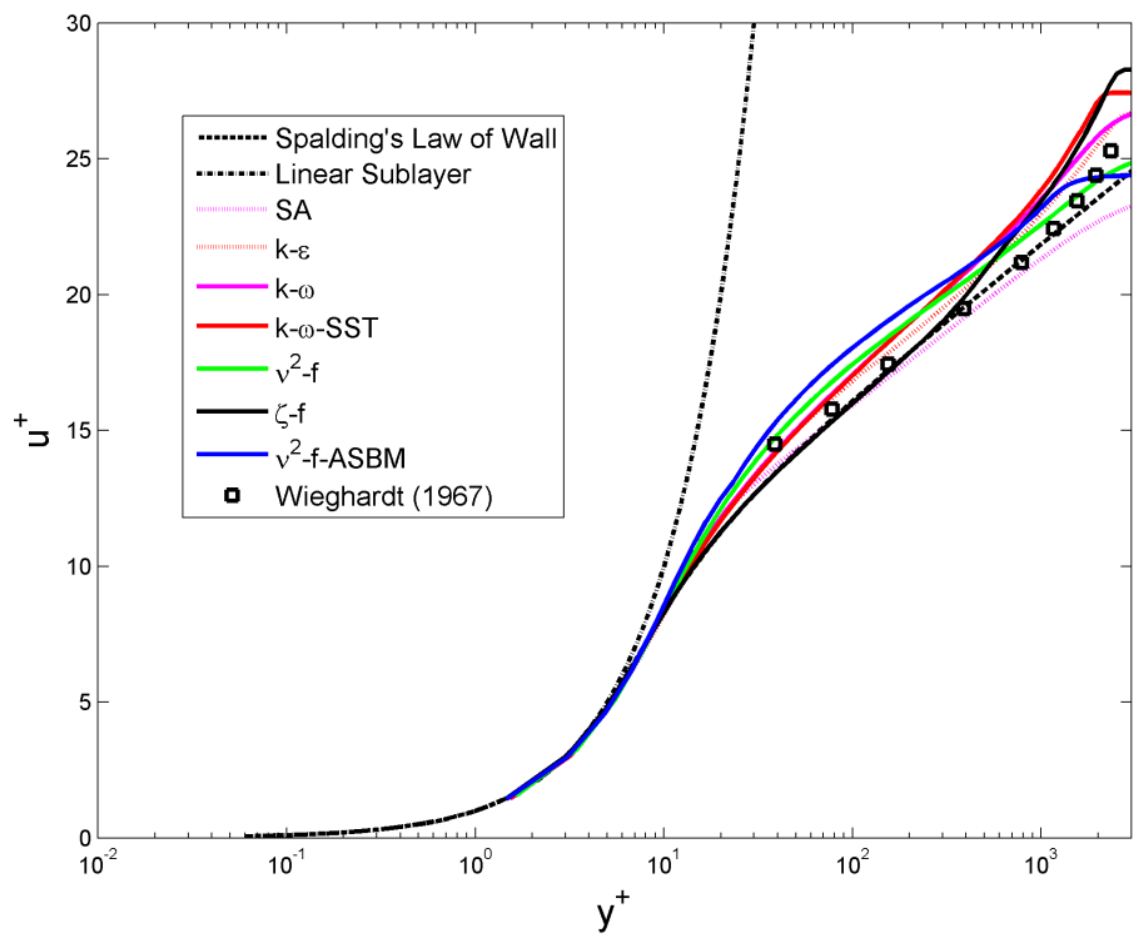

Figure 20. Boundary layer velocity profiles of a turbulent flat plate produced by various turbulence models compared with experimental data. 


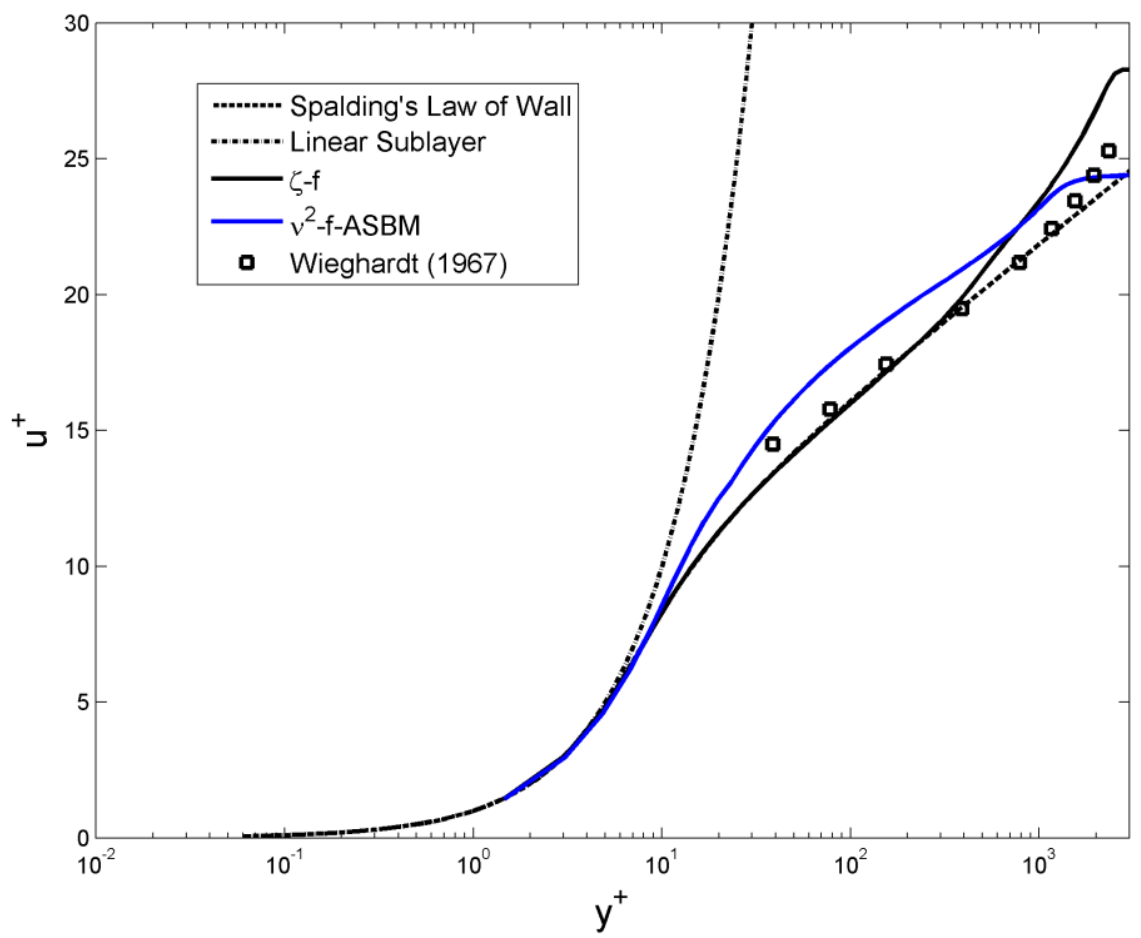

Figure 21. Boundary layer velocity profiles of a turbulent flat plate produced by $\zeta-f$ and $v^{2}-f-$ ASBM compared with experimental data.

The Reynolds stress data was also examined in order to compare the performance of the ASBM to experimental data by Klebanoff. Reynolds stresses calculated using the Boussinesq approximation, the ASBM as a post processing tool, and the semicoupled ASBM are shown below. Post processing is displayed for the $k-\varepsilon$ model below in Figure 22. The ASBM predicts the Reynolds stresses more accurately for both normal stress components. All stresses, however, are under predicted but have the proper shape. The Boussinesq approximation does not predict the wall normal stress well which is expected due to its formulation. 


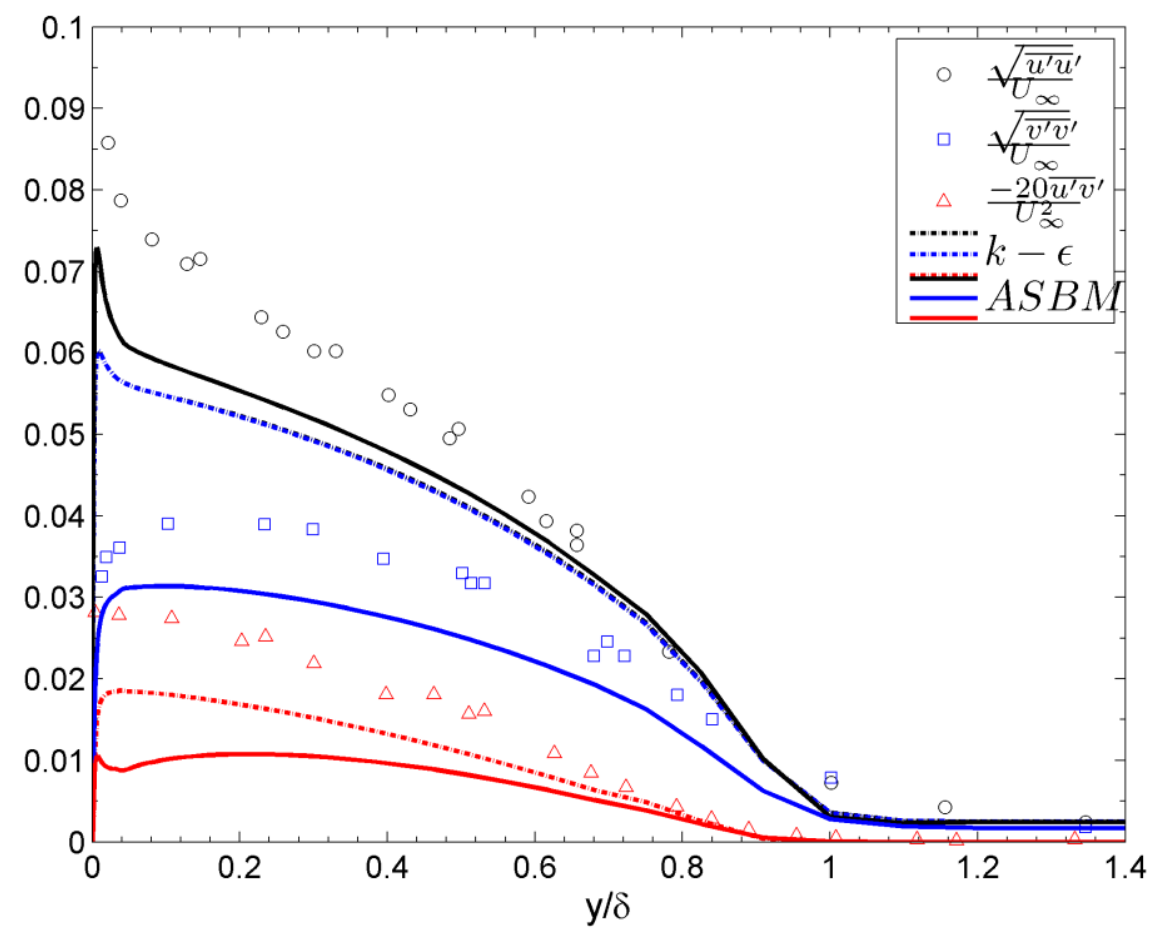

Figure 22. Reynolds stresses at $\operatorname{Re}_{\mathrm{x}}=4.2 \times 10^{6}$ using the Boussinesq approximation and ASBM compared against experimental data of Klebanoff.

For the semicoupled $v^{2}-f$-ASBM the Reynolds stresses are more accurately predicted. The data for the coupled model is displayed below in Figure 23. The Reynolds stresses are still under predicted but seem to merely be off by a scale factor. The shape and distribution is very accurate. A fully coupled model should predict even more accurate Reynolds stresses. 


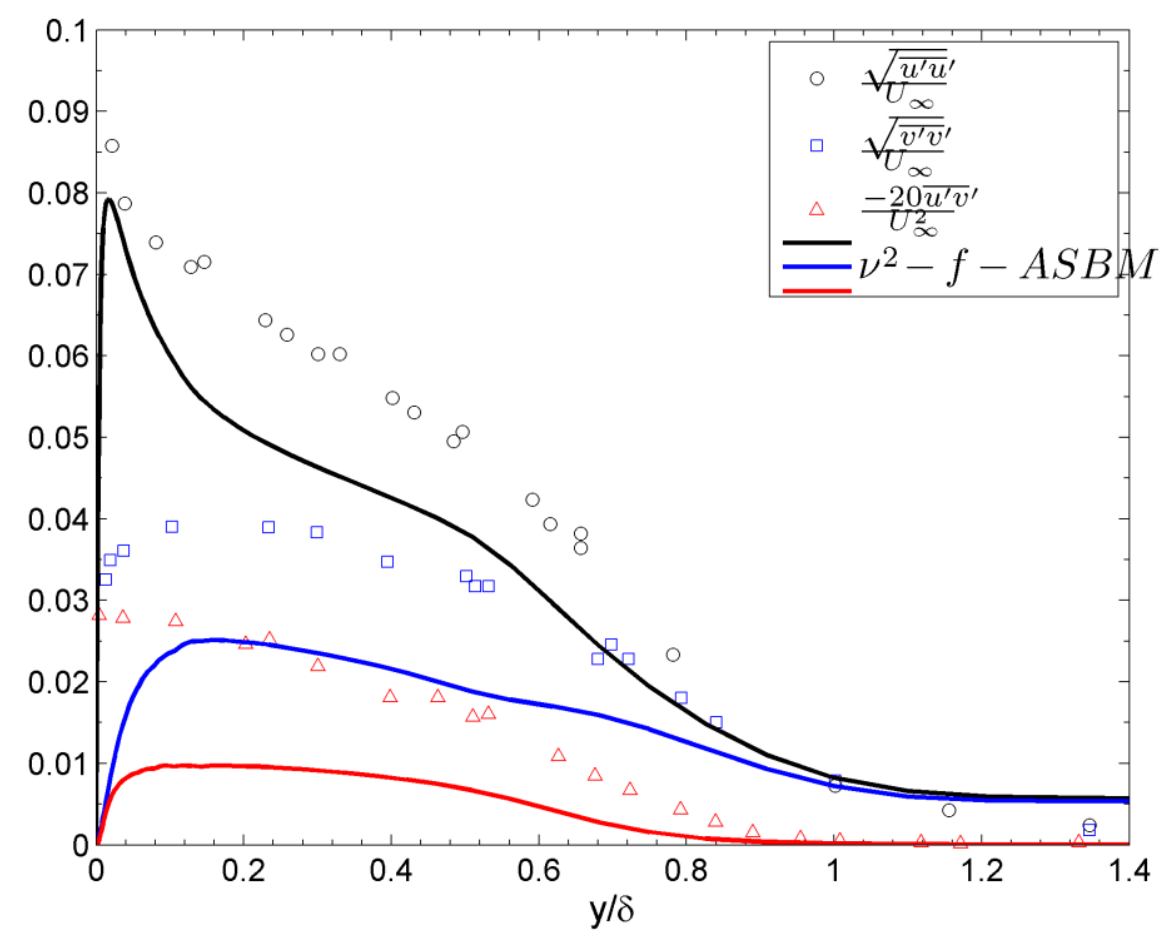

Figure 23. Reynolds stresses at $\operatorname{Re}_{\mathrm{x}}=4.2 \times 10^{6}$ using the $v^{2}$ - $f$-ASBM compared against experimental data of Klebanoff.

Overall, the two models, $\zeta-f$ model and $v^{2}-f$-ASBM, accurately predict flow characteristics of a turbulent flat plate. The $\zeta-f$ model in particular reproduced the boundary layer profile very accurately and generally seemed to predict better than models using less equations, i.e. SA, $k-\varepsilon, k-\omega$. It even produced better predictions than the $k-\omega$ SST. As compared to the $v^{2}-f$ model, the $\zeta-f$ model did predict more accurate skin friction coefficients based on experiment and produced boundary layer profiles closer to experiment. The performance of the $v^{2}-f$-ASBM, however, was comparable to the twoequation models used for comparison. Its prediction of Reynolds stresses, even as just a post processing tool, was more accurate which is promising. To obtain better results, a fully coupled model seems necessary. This is discussed in the Future Work section. 


\section{B. Backward Facing Step}

The canonical backward facing step problem exemplifies turbulence models' abilities to capture separation and reattachment phenomena. Each of the turbulence models discussed as comparison models as well as the implemented $\zeta$-f model and $v^{2}-f$-ASBM were evaluated in this flow type. Specifically, the separation pattern, reattachment length, velocity profiles, and Reynolds stress data has been examined.

The cases were solved with FLUENT's pressure based solver. The pressure-velocity coupling scheme was utilized in order to reach a converged solution. The solution required low courant numbers during the separation bubble formation for all turbulence models used. After initial formation using a first order upwind discretization scheme, a second order upwind scheme was used for all solutions. The solutions were allowed to converge well past dropping residuals in order to ensure proper solutions in a somewhat unsteady flow type.

As discussed earlier, a step height Reynolds number of 5000 was used for all flow calculations and the mesh was created accordingly. The computational domain consists of an inlet section of length $3 h$ and a height of $5 h$ where $h$ is the step height. A sudden expansion of $h$ occurs after the inlet section. This outlet section has a height of $6 h$ and extends for a length of $40 h$ to ensure that the outlet boundary condition has no effect on the separation and reattachment region. In the vertical direction there are 128 cells of which 70 are placed within the step height. The cells are bunched toward the wall with a growth ratio of 1.01-1.025 to ensure that the first cell has a $y^{+}$less than 1.0. 
The $\zeta-f$ model was initialized from a fully converged $k$ - $\varepsilon$ solution and the $v^{2}-f$-ASBM was initialized from a fully converged $v^{2}-f$ solution for stability purposes. The stability of both models is discussed more in depth in a later section.

The separation bubble of each of the comparison turbulence models is displayed below in Figure 24 and Figure 25. The Spalart-Allmaras (SA) model in particular fails to predict a proper recirculation region. Also, each of the models predicts the secondary recirculation region. The secondary recirculation region is extremely exaggerated in the standard $k-\omega$ model and the $k-\omega$-SST model, however.

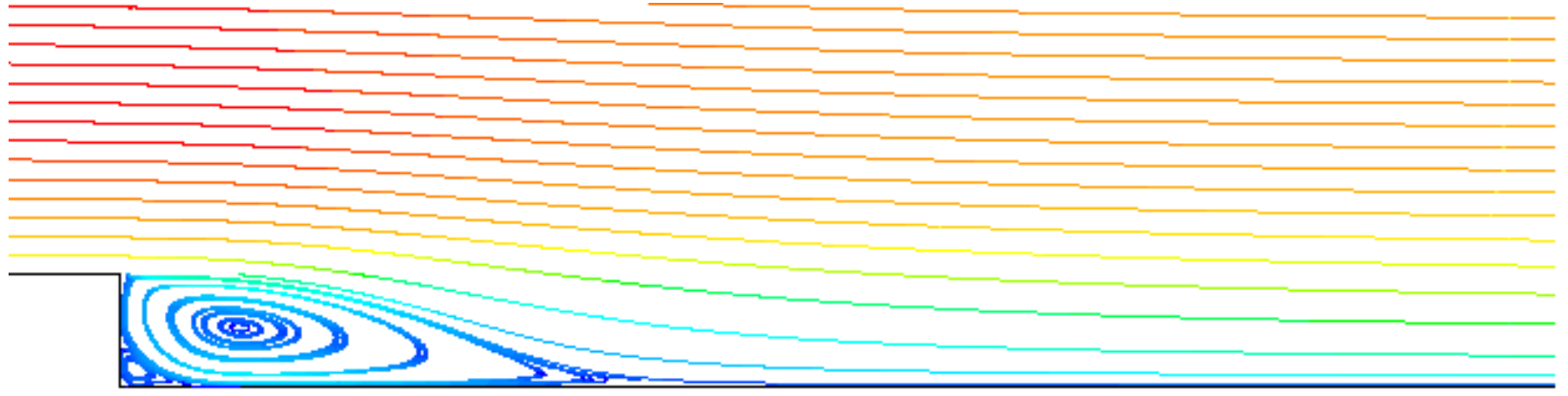

(a)

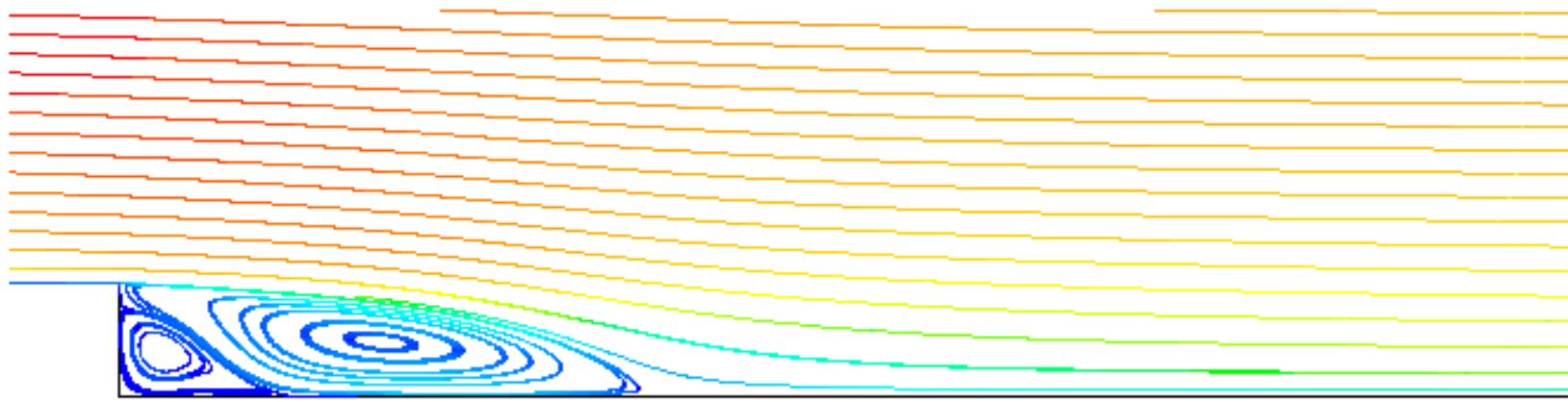

(b)

Figure 24. Separation region for the $k-\varepsilon$ model (a), and the $k-\omega$ model (b). 


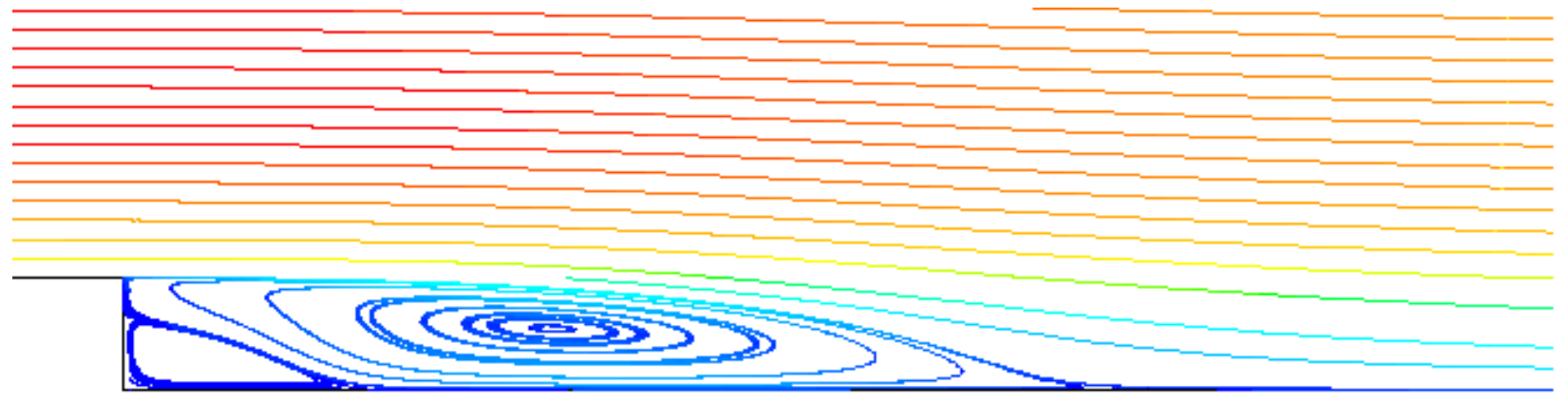

(a)

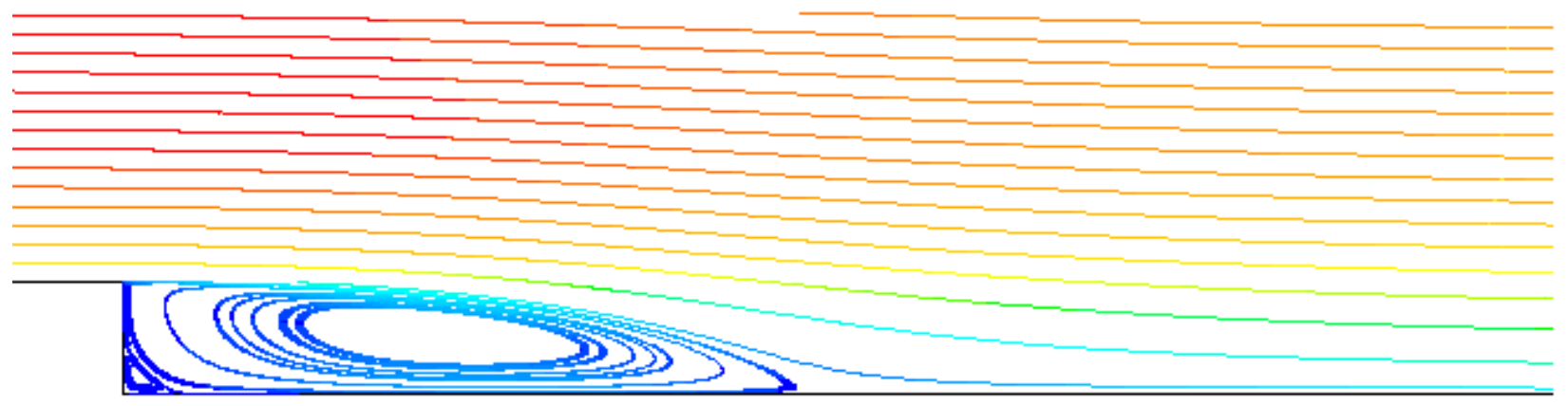

(b)

Figure 25. Separation region for the $k$ - $\omega$-SST model (a), and the $v^{2}-f$ model (b).

The recirculation region of the $\zeta-f$ model and $v^{2}-f$-ASBM are presented below in Figure 26 and Figure 27 respectively. The recirculation region flow characteristics are predicted accurately for both implemented models. Also, both predict the secondary recirculation region accurately without exaggerated size. The region is resolved well.

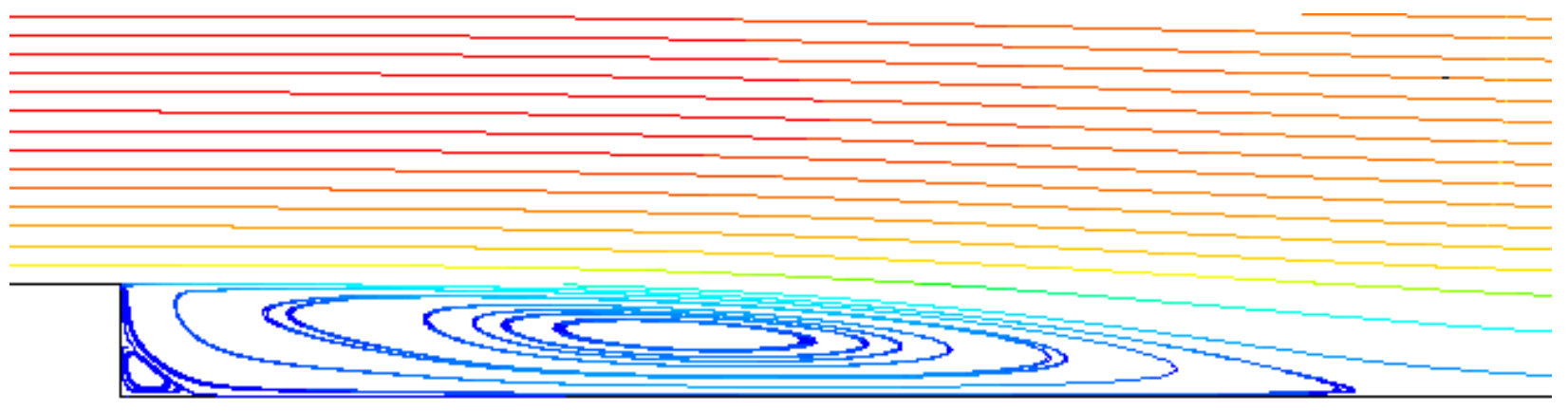

Figure 26. Separation region for the $\zeta-f$ model. 


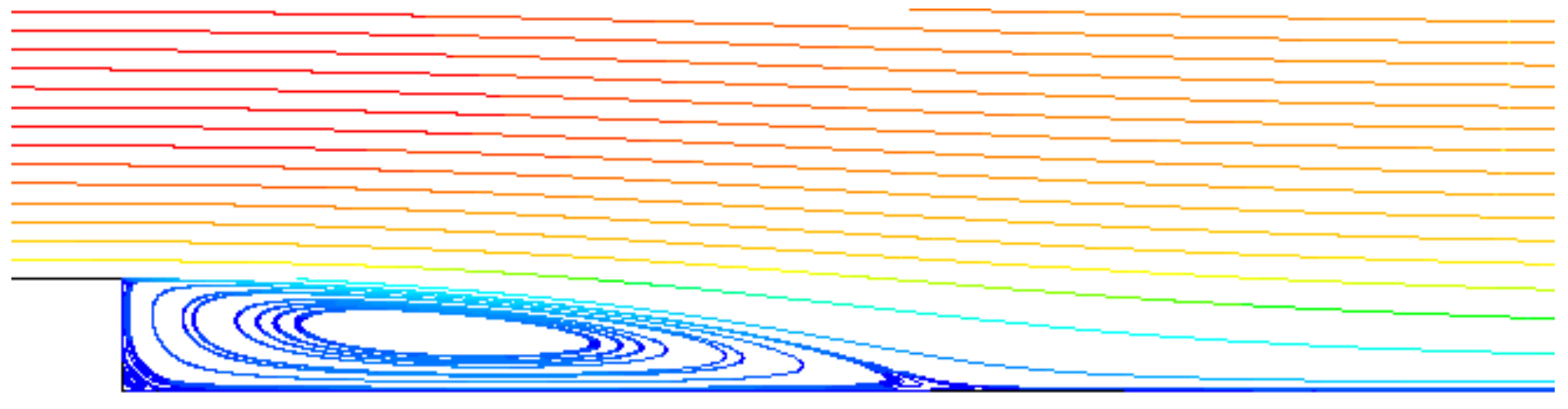

Figure 27. Separation region for the $v^{2}$-f-ASBM.

The separation region is generally characterized by the reattachment length. The reattachment length is determined by the point at which the wall shear stress recovers from zero. Jovic and Driver determined the reattachment length for $\operatorname{Re}_{h}=5000$ from experimental and DNS data to be $X_{r}=6.00 h$ with the experimental data having an uncertainty of $0.15 h$. The reattachment length of both the comparison models and the implemented models are displayed below in Table 12.

Table 12. Comparison of reattachment length to experimental data.

\begin{tabular}{|c|c|c|}
\hline \multirow{2}{*}{ Model } & $\begin{array}{c}\text { Reattachment Length } \\
\left(X_{r} / h\right)\end{array}$ & $\begin{array}{c}\text { Percent Difference } \\
(\%)\end{array}$ \\
\hline Experiment & 6.00 & 0.00 \\
\hline SA & 2.60 & -56.67 \\
\hline$k-\varepsilon$ & 5.25 & -12.50 \\
\hline$k-\omega$ & 5.18 & -13.67 \\
\hline$k-\omega-$ SST & 9.24 & 54.00 \\
\hline$v^{2}-f$ & 6.54 & 9.00 \\
\hline$\zeta-f$ & 11.69 & 94.83 \\
\hline$v^{2}-f$-ASBM & 7.20 & 20.00 \\
\hline
\end{tabular}

Of the models used, the $v^{2}-f$ model predicted the reattachment length most accurately. It also accurately predicted the secondary separation bubble. The $v^{2}$-f-ASBM extended the region slightly while maintaining accurate prediction of the secondary bubble. The $\zeta-f$ model over predicted the reattachment length significantly but still produced an accurate secondary bubble. The $k-\omega$ model predicts accurately but has a largely exaggerated 
secondary recirculation region. The $k-\omega$-SST secondary bubble is largely exaggerated as well. It is interesting to note the difference between performance of the $\zeta-f$ model and the $v^{2}-f$ model since the $\zeta-f$ model is mathematically nearly identical to the $v^{2}-f$ model.

The velocity profiles throughout the separation region can also be examined and compared against experiment. The streamwise velocity profiles for $v^{2}-f, \zeta-f$, and $v^{2}-f$ ASBM compared against experimental data are shown below in Figure 28, Figure 29, and Figure 30. The other models will not be examined further due to poor prediction compared to the $v^{2}-f$ model.

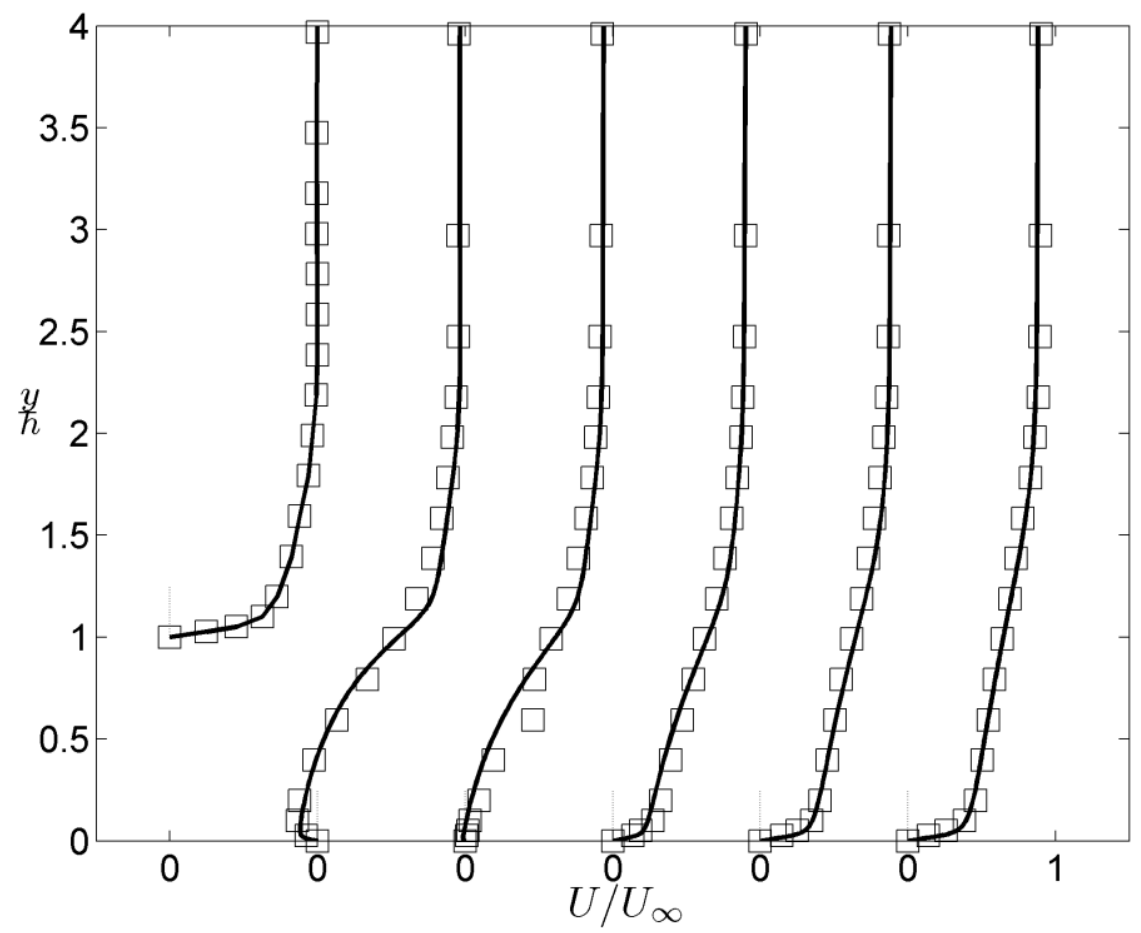

Figure 28. Streamwise velocity profiles at $x / h=-3,4,6,10,15$, and 19 for $v^{2}-f$ (solid line) compared against experimental data $(\square)$. 


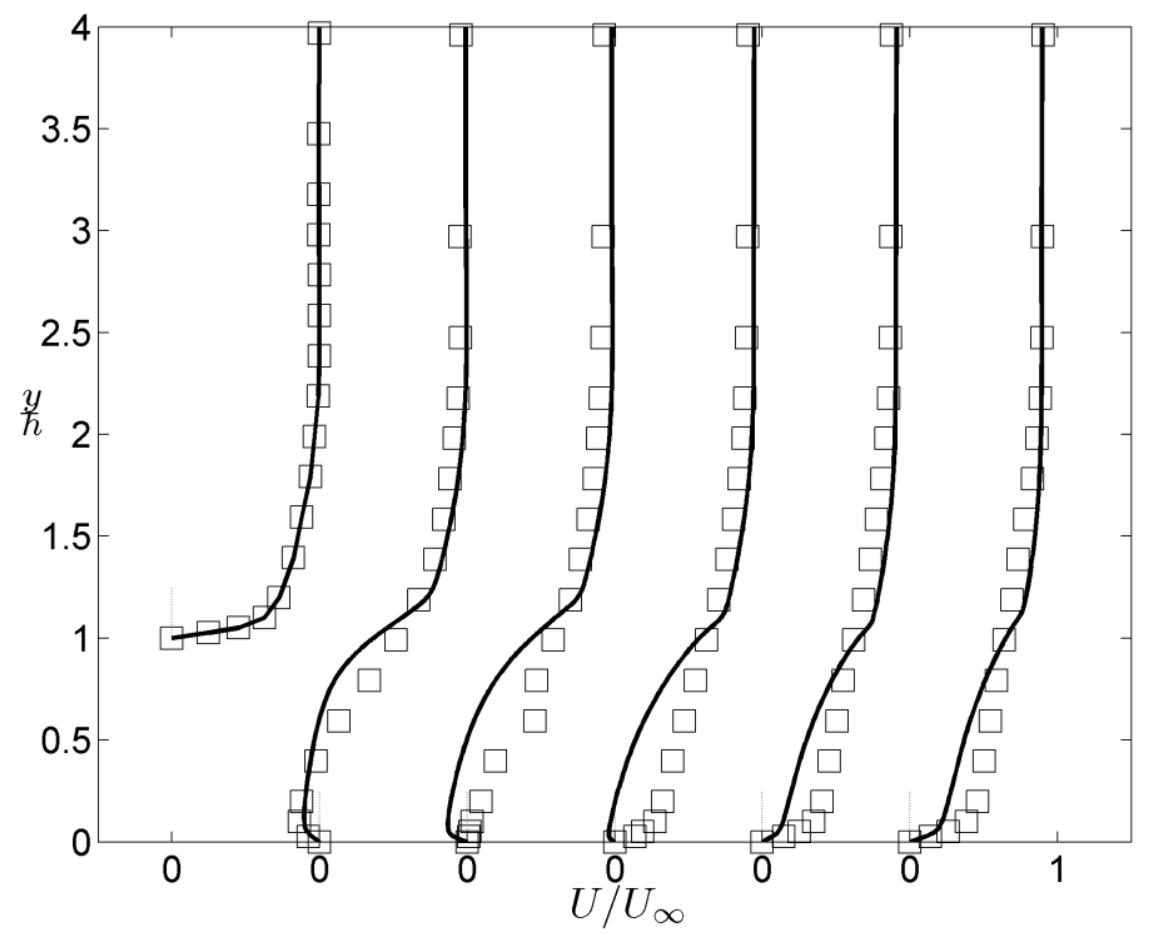

Figure 29. Streamwise velocity profiles at $x / h=-3,4,6,10,15$, and 19 for $\zeta-f$ (solid line) compared against experimental data $(\square)$.

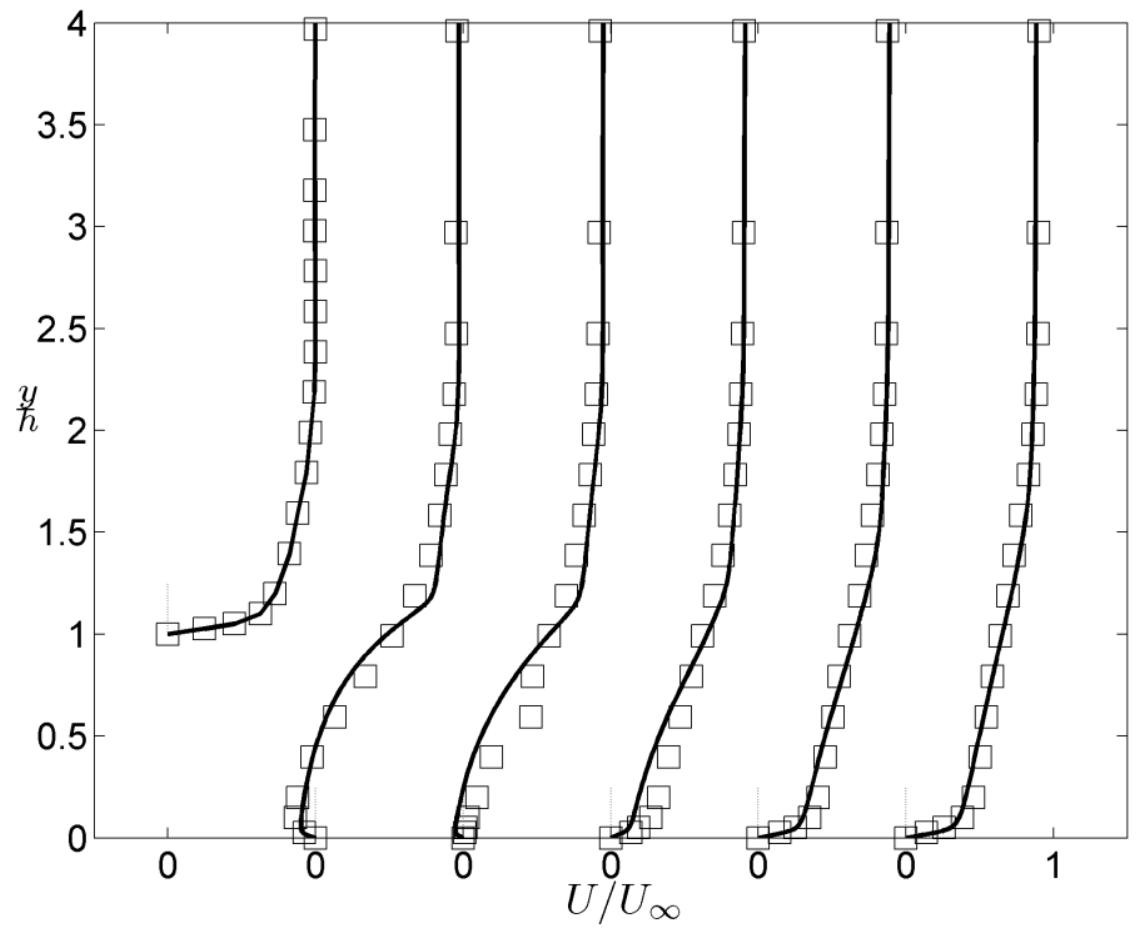

Figure 30. Streamwise velocity profiles at $x / h=-3,4,6,10,15$, and 19 for $v^{2}$-f-ASBM (solid line) compared against experimental data $(\square)$. 
The velocity profiles for each of three models match well with the experimental data. The $v^{2}-f$ model results in the most accurate prediction which is to be expected based on reattachment length data. In contrast, the $\zeta-f$ model predicts the reversed flow near the step very well but creates too large of a stagnation region. The $v^{2}-f$-ASBM predicts very similarly to the $v^{2}-f$ model which is expected due to its formulation. In the shear layer between the separated region and mean flow, each of the models predicts well as compared with the experimental data. Prediction of the shear region is slightly worse for the $\zeta-f$ model, however.

The Reynolds stresses can also be examined at the sample stations as predicted by the Boussinesq approximation used in the $v^{2}-f$ model, the ASBM as a post processing tool on the $v^{2}-f$ model, and the semicoupled $v^{2}-f$-ASBM. The Reynolds normal stresses and the Reynolds shear stress are shown for each of the prediction methods in Figure 31, Figure 32, and Figure 33. 

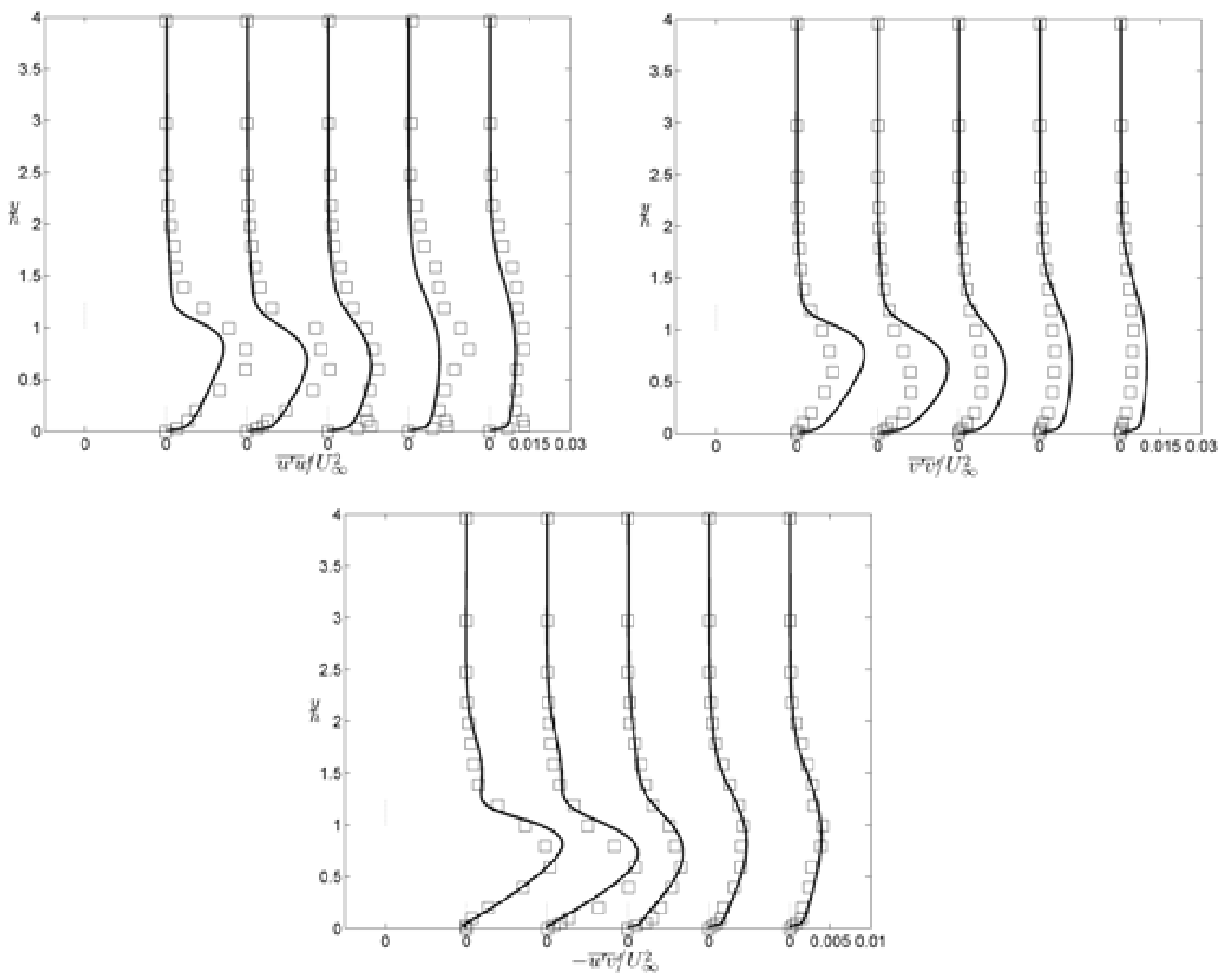

Figure 31. Backward facing step Reynolds stresses determined by the Boussinesq approximation with the $v^{2}-f$ model (solid lines) compared against experimental data ( $\square$ ). 

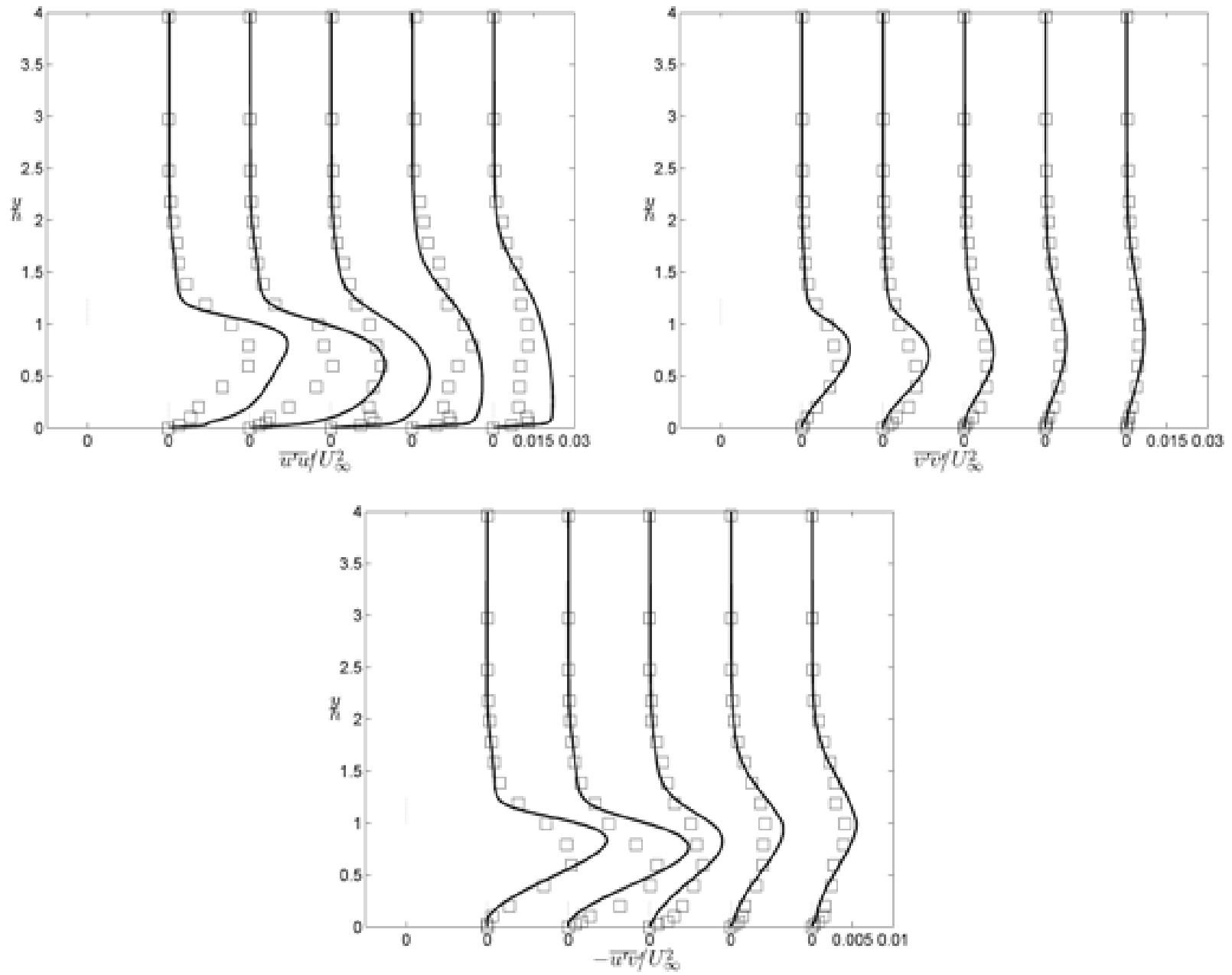

Figure 32. Backward facing step Reynolds stresses determined by the ASBM with the $v^{2}-f$ model (solid lines) compared against experimental data ( $\square$ ). 

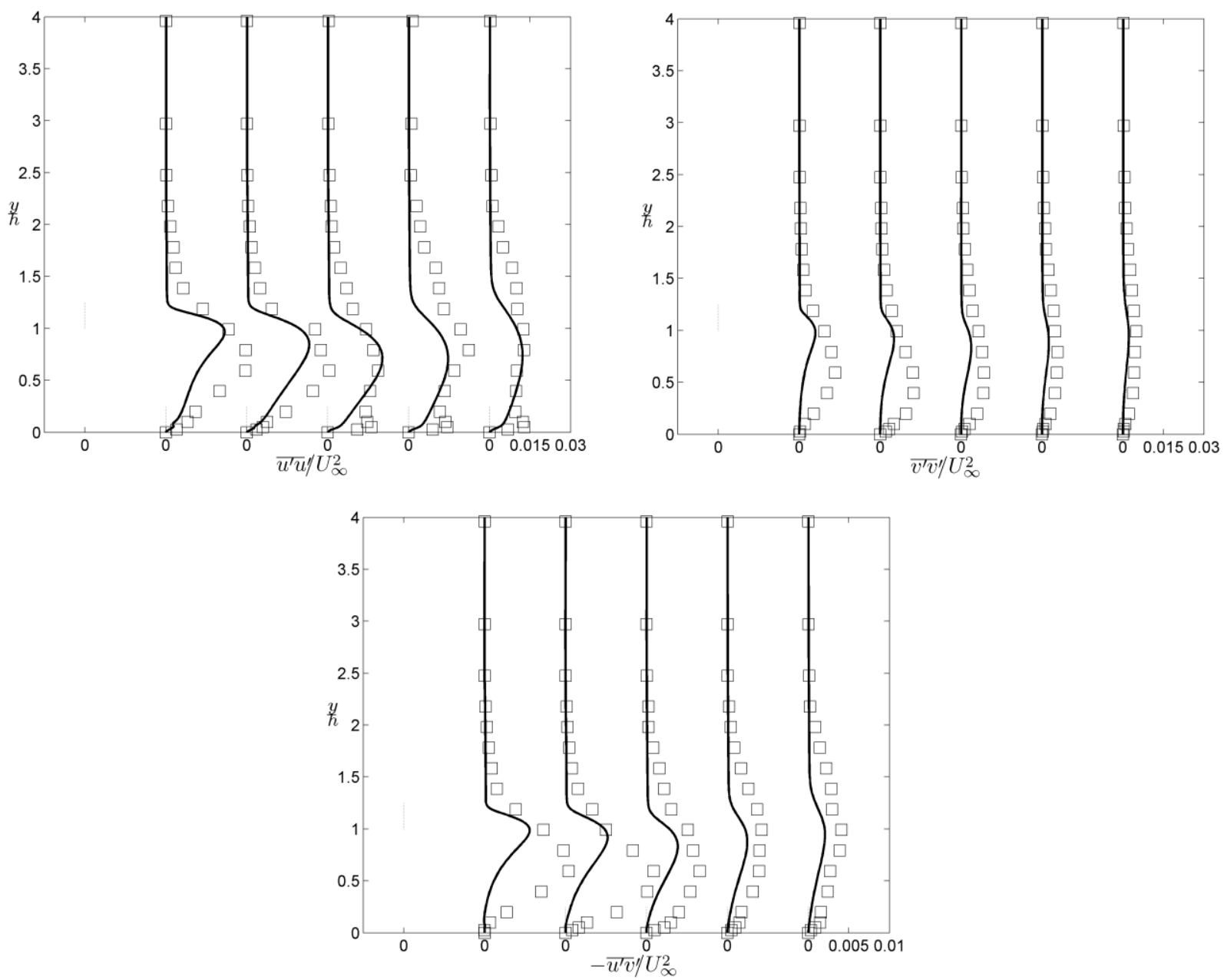

Figure 33. Backward facing step Reynolds stresses determined by the $v^{2}$-f-ASBM model (solid lines) compared against experimental data $(\square)$.

The Reynolds stresses are generally predicted poorly in complex flow regimes using the Boussinesq approximation. It is apparent in Figure 31 that the streamwise Reynolds stress is under predicted near to the step while the wall normal Reynolds stress is over predicted. As the flow becomes more and more isotropic toward the reattachment point, the Reynolds stress predictions become better. In the shear layer between the recirculation region and mean flow, the normal Reynolds stresses are predicted poorly as well. The shear Reynolds stress is predicted surprisingly well. Using the ASBM as a post processing tool produced larger values for the streamwise and wall normal Reynolds stresses than the Boussinesq approximation. For the wall normal direction the prediction 
is slightly better. The streamwise values are off by a similar scale, however. The shear layer is predicted more accurately with the ASBM. The shear component is surprisingly slightly less accurate for the ASBM. When the ASBM is coupled with the $v^{2}-f$ model, the predictive capabilities of the model seem to increase for the streamwise Reynolds stresses especially in the shear region. The stresses are generally under predicted throughout the recirculation region, however. This is partially due to the less accurate reattachment length for the semicoupled model. It seems that in order to accurately predict the Reynolds stresses using the ASBM, full coupling of the model with the mean flow would be necessary. Overall, however, the predictive abilities of the standard $v^{2}-f$ model seem better than that of the semicoupled model. The ASBM does, however, predict the Reynolds stresses more accurately when used as a post processing tool.

\section{S3H4 2D Hill}

The S3H4 2D hill is a sinusoidal hill represented by the following equation.

$$
y=\frac{H}{2}\left\{1+\cos \left[\left(\frac{\pi}{2}\right)\left(\frac{25 x}{H}\right)\right]\right\}
$$

where the length is 25 times the height. This geometry is consistent with the geometry defined by Kim et al. A boundary layer height Reynolds number of 7 was used for CFD to mimic the experiment. The geometry and case is further described in section 1.B.3.

The cases were solved with FLUENT's pressure based solver with a similar set up to the backward facing step case. The pressure-velocity coupling scheme was utilized in order to reach a converged solution. Low courant numbers were used until the flow was well defined over the hill. After initial formation using a first order upwind discretization scheme, a second order upwind scheme was used for all solutions. 
Experimental velocity profile data around the hill was compared against the velocity profiles generated using each of the comparison turbulence models and the implemented turbulence models. This comparison is displayed below in Figure 34 for the comparison models. The experimental data does not show a separation region but the SpalartAllmaras and $v^{2}-f$ model do. 

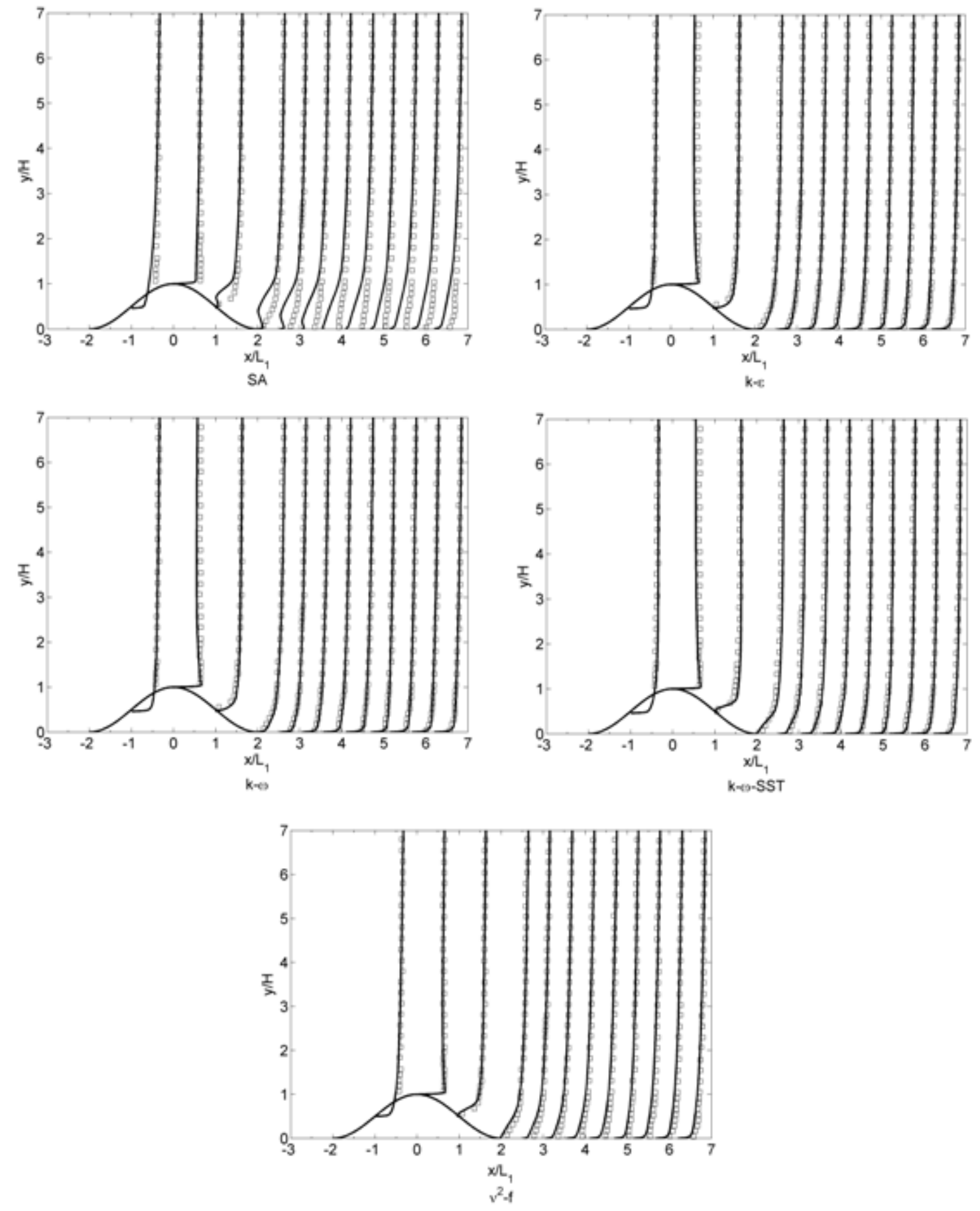

Figure 34. S3H4 2D hill velocity profiles for various turbulence models (solid line) compared against experimental data $(\square)$.

The two equation comparison models perform well on the $\mathrm{S} 3 \mathrm{H} 4$ hill while the others show separation where it should not be present. Similar to the $v^{2}-f$ model, the $\zeta-f$ model 
and $v^{2}$-f-ASBM show a separation region. The separation region for the two implemented models is slightly enlarged compared to the $v^{2}-f$ results. It is interesting to note that the models also increased the separation length as compared to the $v^{2}-f$ model in the backward facing step case as well. Unfortunately this separation is not desired in this case. Since both models are based on the $v^{2}-f$ model, it is not surprising, however, that similar difficulties in capturing the high streamline curvature would exist. The $\zeta_{-} f$ model's and $v^{2}$-f-ASBM's performance is sub par for this validation case. The model results are shown below in Figure 35 and Figure 36.

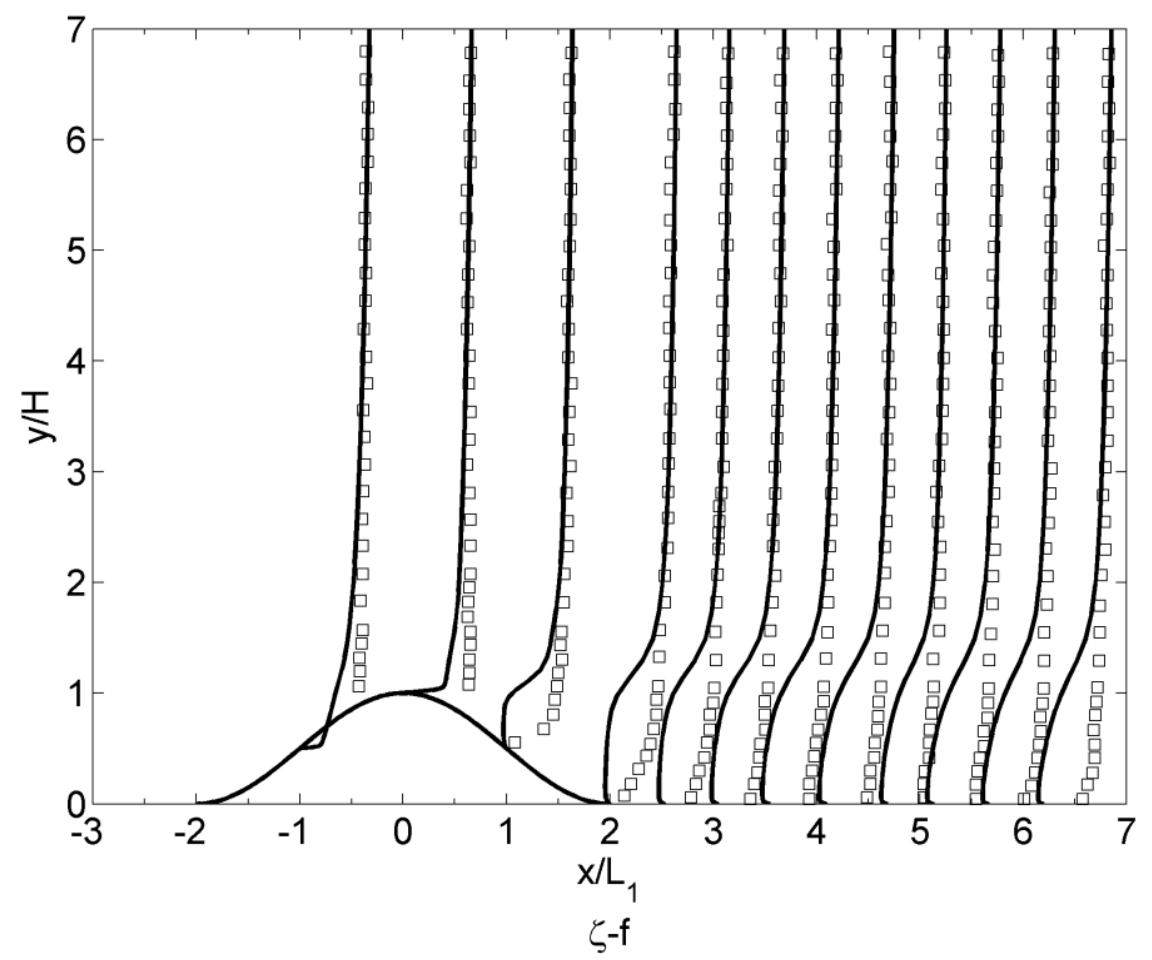

Figure 35. S3H4 2D hill velocity profiles for the $\zeta-f$ model (solid line) compared against experimental data ( $\square$ ). 


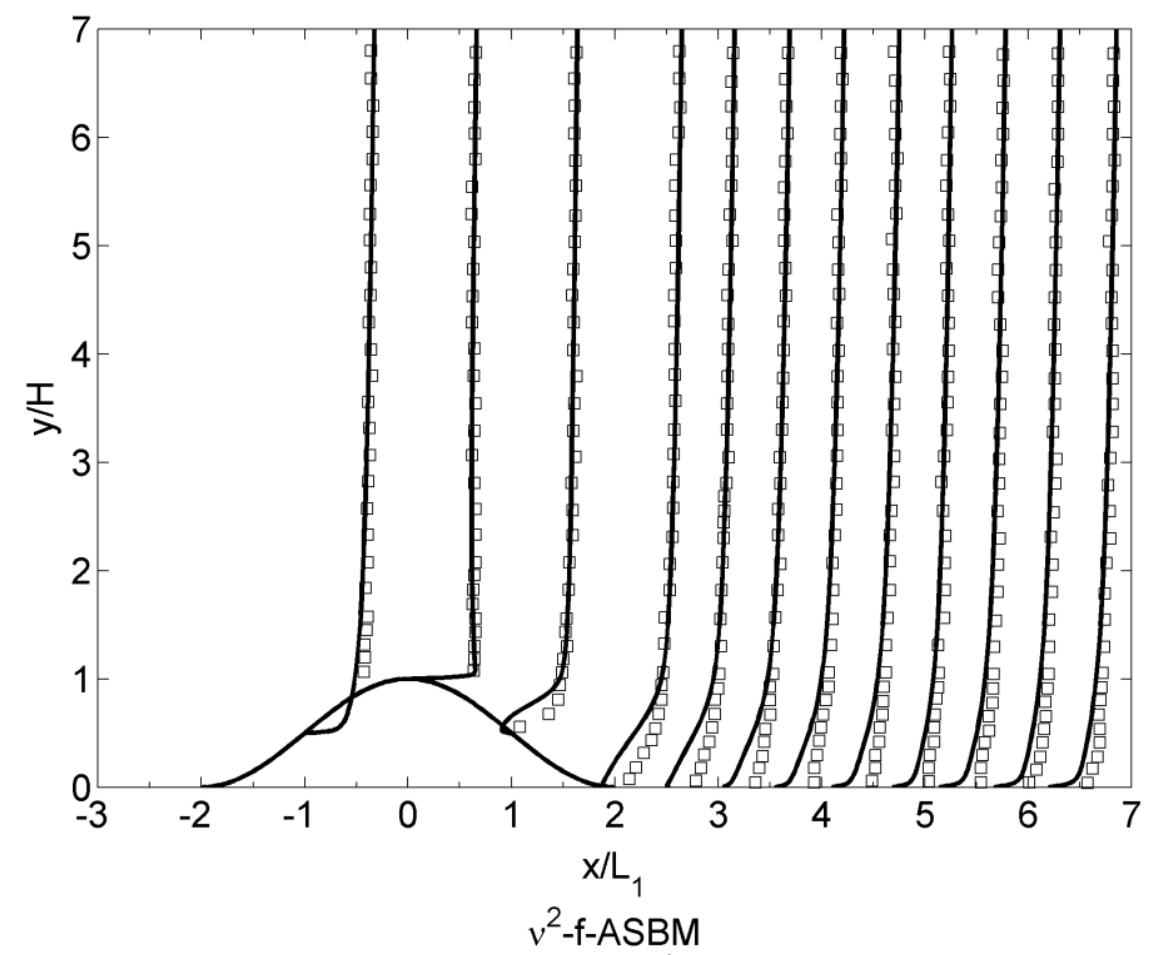

Figure 36. S3H4 2D hill velocity profiles for the $v^{2}-f$-ASBM (solid line) compared against experimental data $(\square)$.

\section{Stability and Robustness}

Both of the implemented models require additional computing power as compared to most general application turbulence models. This is due to the addition of transport equations for the case of the $\zeta_{-} f$ model and also the addition of a computationally expensive algebraic calculation for the ASBM. As compared to built in two equation models in FLUENT, the $\zeta-f$ model takes between $20-30 \%$ more computing time while the $v^{2}$-f-ASBM requires 50-60\% more computing time per iteration. The $\zeta-f$ model, however, was created to improve stability and robustness while the ASBM is still in development stages.

The $\zeta-f$ model is meant to perform similarly to the $v^{2}-f$ model with increased stability and robustness. Part of the robustness scheme meant to improve the $\zeta-f$ model was the zero wall boundary condition. The $v^{2}-f$ model used also has a zero wall boundary 
condition, however. In order to compare stability, the residuals of x-velocity, $y$-velocity, and continuity are compared for the $\zeta-f$ model and $v^{2}-f$ model as computed during flat plate calculation. The residual plots are displayed below in Figure 37 and Figure 38.

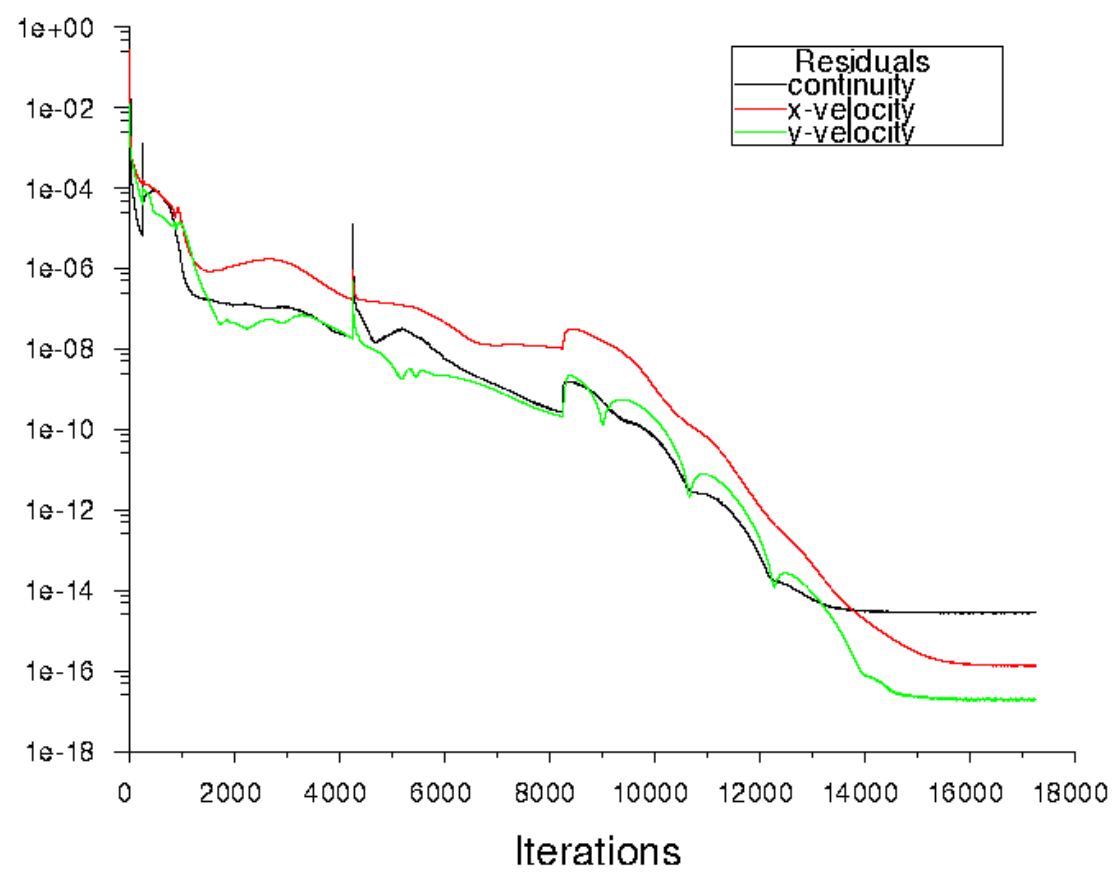

Figure 37. Residuals of $v^{2}-f$ model for turbulent flat plate.

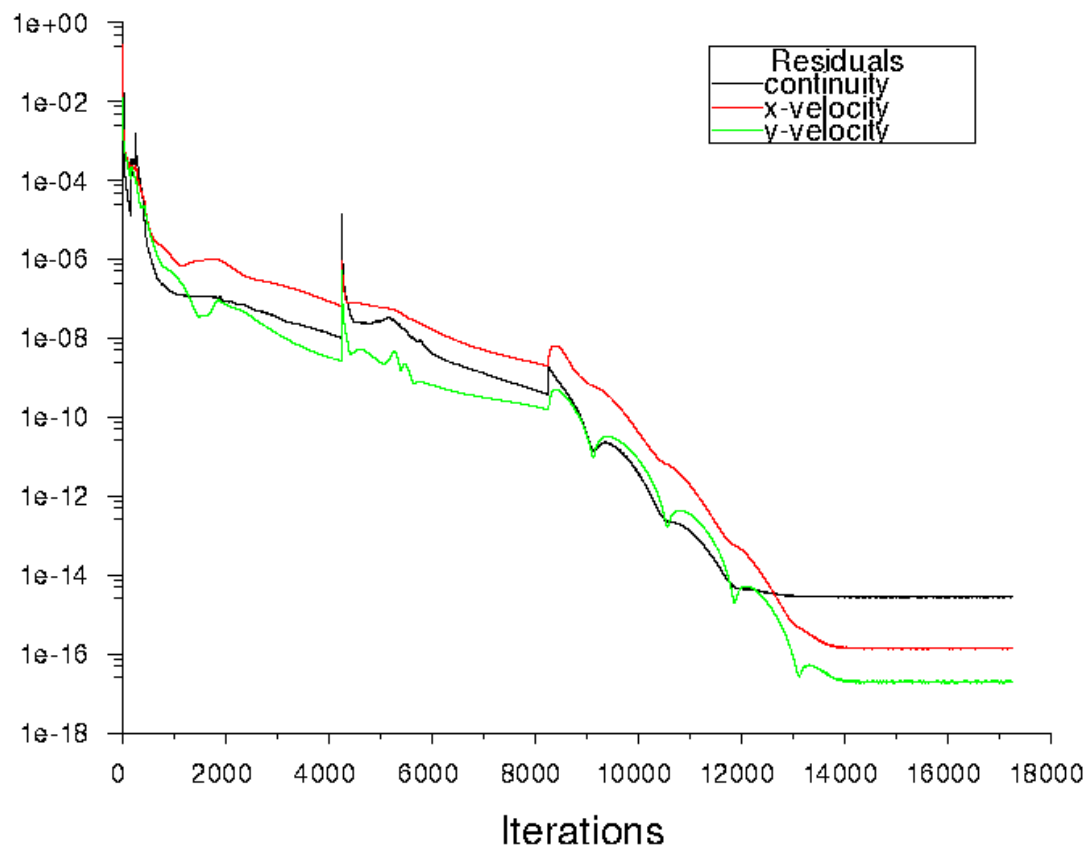

Figure 38. Residuals of $\zeta-f$ model for turbulent flat plate. 
Examining the residual plots, it is apparent that the residuals of the $\zeta-f$ model are lower and also drop faster than that of the $v^{2}-f$ model. This is most apparent with the $\mathrm{x}$ velocity residual after under relaxation factors have been raised at about 8000 iterations. All of the residuals also level out sooner for the $\zeta-f$ model. The improvement is small but still apparent. While improvement is shown for the flat plate, it is important to note that during calculation of the backward facing step, the $\zeta-f$ model had no noticeable improvement over the $v^{2}-f$ model. Stability benefits were not apparent for that test case. Overall, the $\zeta-f$ model shows similar characteristics for stability and robustness as the $v^{2}-f$ model which was used.

Stability and robustness of the ASBM, however, are lacking. Aside from the extra computational expense, the ASBM, and all algebraic models for that matter, have no memory with regard to flow characteristics. Thus response to velocity gradients is instantaneous and purely reactive. As a result, the model has problems resolving flow features where velocity gradients are changing rapidly. This is especially apparent near the inlet during flow formation. Thus resolving the flow features requires special attention during calculation to avoid divergence. Unfortunately, this is a deterrent for use in complex flows where the benefit of the model might outweigh its computational expense. In addition to robustness issues with the model, the elliptic relaxation function used for the blockage parameter has stability issues within the FLUENT solver.

The elliptic relaxation function's stability, a Helmholtz equation, is highly dependent on the length scale used. More specifically, the stability decreases rapidly when the Kolmogorov scale limit is decreased, i.e. decreasing $C_{v}$. This is synonymous with increasing the wave number of the Helmholtz equation at the Kolmogorov limit. 
Solving a Helmholtz equation at large wave numbers is considered a difficult task in scientific computing and proves difficult with the FLUENT solver. To obtain a solution for the elliptic relaxation function with small values of $C_{v}\left(C_{v}<5\right)$, the under relaxation factor must be dropped below $10^{-2}$. This of course results in slow convergence. To properly use the ASBM with this stability problem, the elliptic relaxation function must be allowed to converge independently to avoid computation expense. After convergence, the additional transport equations of the coupled turbulence model and the ASBM can be implemented. Alternatively, the mesh can be resolved further (well beyond the requirements of the base turbulence model). This, however, creates a much higher computational strain than the other method. 


\section{Conclusion}

Both the $\zeta-f$ model and the ASBM have been implemented in FLUENT and also validated on multiple canonical cases. The $\zeta-f$ model produced improved results for the flat plate as compared to all two-equation or less turbulence models and showed similar predictive capabilities to the $v^{2}-f$ model. The recirculation region in the backward facing step, however, was greatly over predicted. It had difficulties predicting attached flow past the S3H4 2D hill just as the $v^{2}-f$ model. This, however, was expected due to its basis on the $v^{2}-f$ model. The model was also more stable than the $v^{2}-f$ model during calculation of the turbulent flat plate but showed no noticeable improvement in robustness for the more complex backward facing step. The semicoupled (linear eddy viscosity model based) $v^{2}$-f-ASBM's predictive capabilities was comparable to the two equation models for the turbulent flat plate case. It performed well for the backward facing step but reduced accuracy as compared to the $v^{2}-f$ model. The model did, however, have problems predicting the $\mathrm{S} 3 \mathrm{H} 42 \mathrm{D}$ hill just as the with the $v^{2}-f$ model. Reynolds stress prediction was generally improved over a flat plate although it seems that coupling the ASBM with the mean flow would be necessary for greater accuracy. This was even more evident in the backward facing step case. The ASBM did present stability issues as well as implementation issues within FLUENT's user defined functions. 


\section{Future Work}

This paper provides the basis for the validation of the ASBM on gradually more

complicated flows. So far, work has been done implementing the $k-\varepsilon-A S B M$ and the $v^{2}-f$ -ASBM turbulence models defined as a user defined function for the flow solver FLUENT. The work presented does not couple the ASBM with the mean flow, however. This coupling should provide more accurate prediction of flow. A significant amount of work has been committed and the frame work for implementation has been coded. Stability and divergence issues have made this implementation difficult, however. It seems that FLUENT's user-defined functions have no method to adjust the mean flow coupling smoothly. Approximating the Boussinesq hypothesis gradients within a small margin throughout the whole flow regime has proven difficult. Small inconsistencies between the approximation and gradients used by FLUENT introduce improper flow characteristics and eventually leads to divergence. Preliminary results of turbulent flat plate flow for the fully coupled $k-\varepsilon$-ASBM are presented below in Figure 39 . This solution is not fully converged and only first order. If it is iterated further, pockets of enlarged turbulent kinetic energy form near the inlet where larger flow gradients are present. It does, however, show promising results. The laminar sublayer and overlap layer match well with theoretical data. In the log layer, however, the slope is not predicted accurately. The Reynolds stresses can also be examined and are shown in Figure 40. Since the flow is coupled with the ASBM, results are more indicative of the ASBM's predictive capabilities. The Reynolds stresses are predicted quite well. 


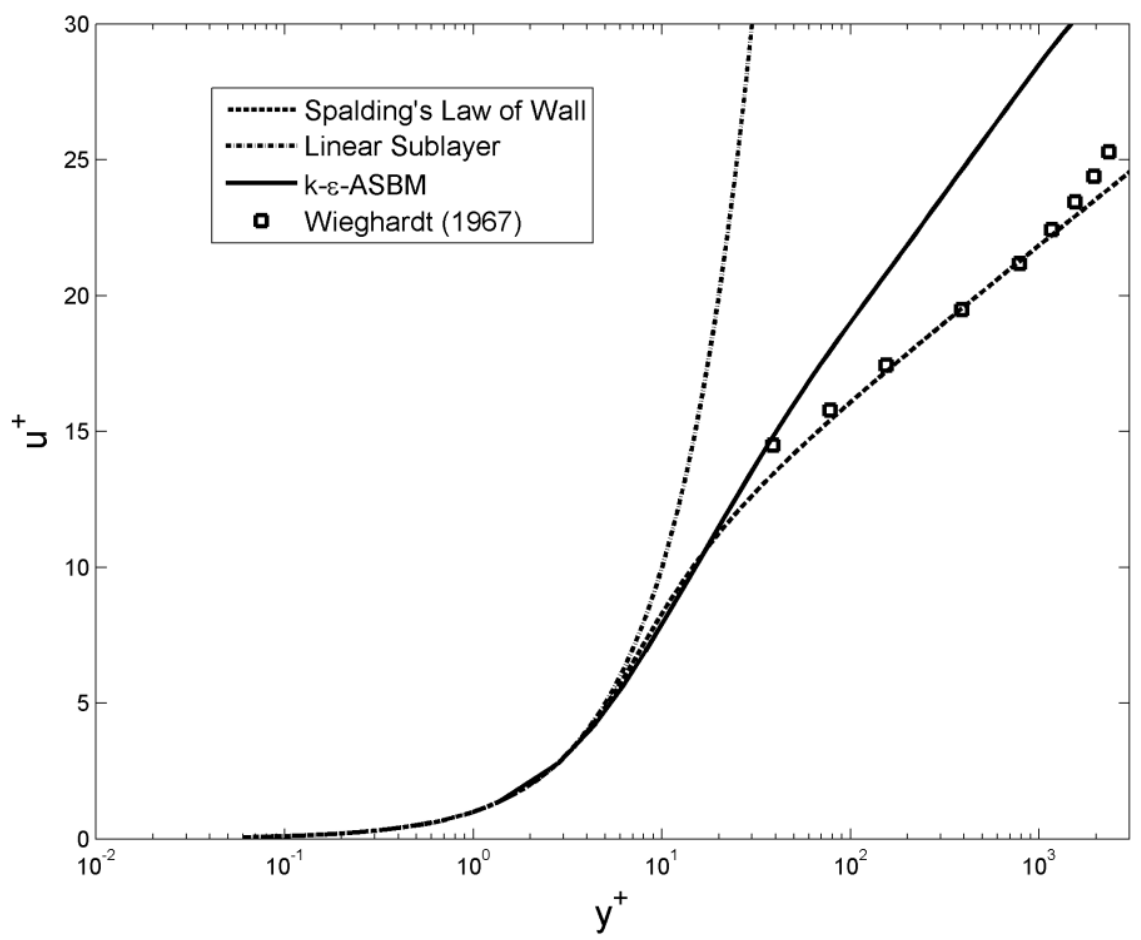

Figure 39. Boundary layer velocity profiles of a turbulent flat plate produced by $k-\varepsilon-A S B M$ compared with experimental data.

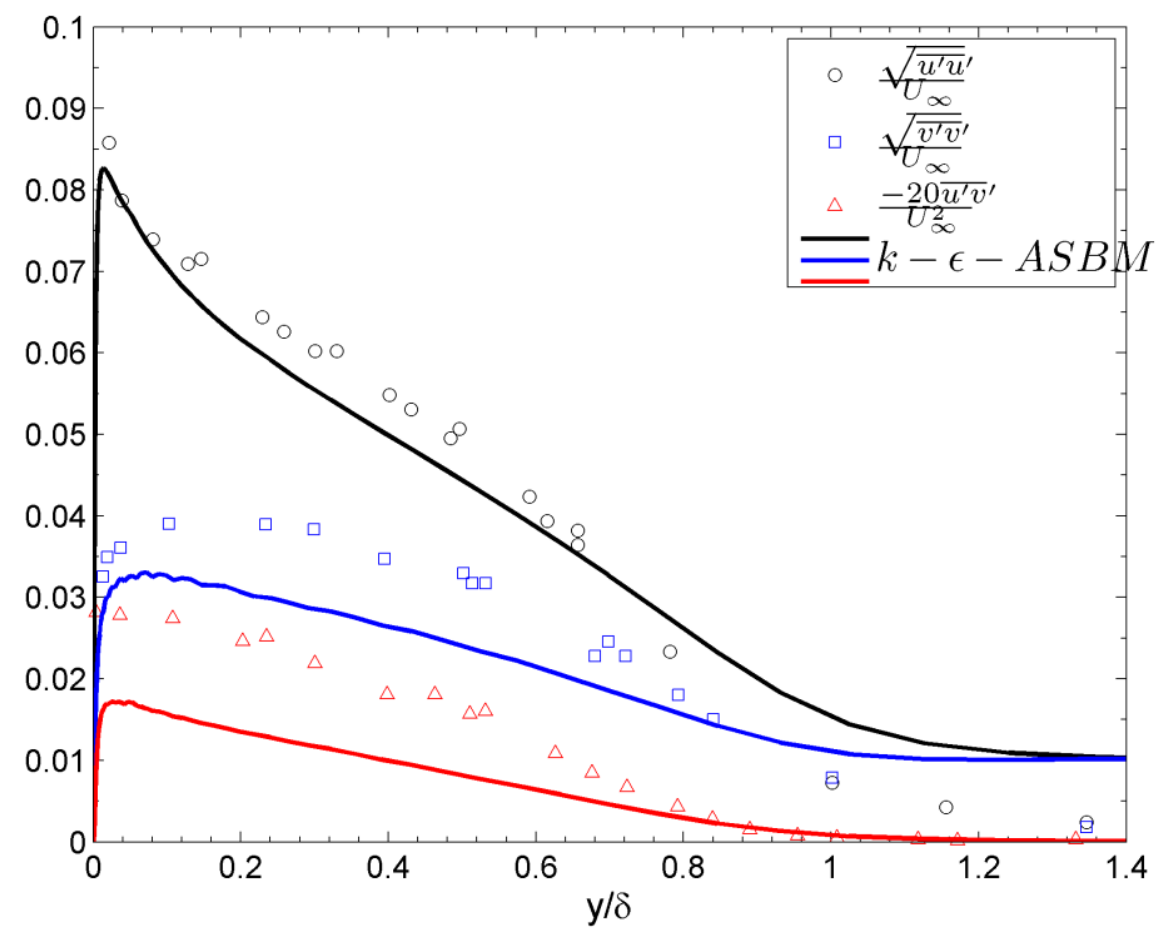

Figure 40. Reynolds stresses at $\operatorname{Re}_{\mathrm{x}}=4.2 \times 10^{6}$ using the fully coupled $k$ - $\varepsilon$-ASBM compared against experimental data of Klebanoff. 
Future work will include attempts to fully couple the ASBM based on this initial promising work. Improvements in FLUENT's UDF macros may be necessary to fully couple the ASBM, however. 


\section{References}

Akylas, E., Langer, C. A., Kassinos, S. C., and Demosthenous, E., "On the linear stability of turbulent plane strain flow in a rotating frame.” Physics of Fluids, Vol, 19, No. 7, 2007.

ANSYS $^{\circledR}$ FLUENT 12 User Guide, ANSYS, Inc.

Baldwin, B.S., and Lomax, H., "Thin layer approximation and algebraic-model for separated turbulent flows." AIAA, pg. 78-257, 1978.

Bardina, J.E., Huang, P.G., and Coakley, T.J., "Turbulence modeling validation, testing, and development.” NASA TM 110446, 1997.

Bell, B., "Turbulent flow case studies." Fluent Services Center, 2003.

Benton, J.J., "Evaluation of $v^{2}-f$ and ASBM turbulence models for transonic aerofoil RAE2822.”ERCOFTAC Series 14, 2011.

Borello, D. and Orlandi, P., "DNS scrutiny of the $\zeta-f$ elliptic relaxation eddy-viscosity model in channel flows with a moving wall.” Flow Turbulence Combust, vol. 86, pg. 295-309, 2011.

Breuer, M., Ray, S., Kumar, V., Jovanovic, J. and Durst, F., “Anisotropy invariant Reynolds stress model and its application to fully developed channel flows.” Proc. Appl. Math. Mech., vol. 5, pg. 537-538, 2005.

Davidson, L., Nielsen, P.V., and Sveningsson, A., "Modifications of the $v^{2}-f$ model for computing the flow in a 3d wall jet. Turbulence Heat and Mass Transfer. vol. 4, 2003.

Durbin, P.A., "Near-wall turbulence closure without damping functions." Theoretical and Computational Fluid Dynamics, 3:1-13, 1991.

Durbin, P.A., "Separated flow computations with the $k-\varepsilon-v^{2}$ model." AIAA Journal, vol. 33, no. $4,1995$. 
Haire, S. L., and Reynolds, W. C., "Toward an affordable two-equation structure-based turbulence model.” Technical Report TF-84, Mechanical Engineering Department, Stanford University, 2003.

Hanjalic, K., Popovac, M., and Hadziabdic, M., “A robust near-wall elliptic-relaxation eddyviscosity turbulence model for CFD.” Int. J. Heat Fluid Flow, vol. 25, pg. 1047-1051, 2004.

Heschl, Ch., Sanz, W., and Klanatsky, P., "Implementation and comparison of different turbulence models for three dimensional wall jets with FLUENT." TU Graz, Institute for Thermal Turbomachinery and Machine Dynamics, 2005.

Iaccarino, G., "Predictions of a turbulent separated flow using commercial CFD codes." Journal of Fluid Engineering. vol. 123, pg. 819-828, 2001.

Jovic, S. and Driver, D. M., "Backward-facing tep easurements at low Reynolds number, $\operatorname{Re}_{h}$ =5000.”NASA Technical Memorandum 108807, 1994.

Kalitzin G., Iaccarino G., Kassinos S. C. and Langer C. A., "Combining eddy-viscocity models and the algebraic structure-based Reynolds stress closure." Proceedings of the Summer Program 2004, Center for Turbulence Research, NASA/Stanford Univ, 2004.

Kalitzin, G., Iaccarino, G., and Langer, C. A., "Combining eddy-viscosity models and the algebraic structure-based Reynolds stress closure.” Center for Turbulence Research, NASA/Stanford Univ, 2004.

Kassinos S. C. and Reynolds W. C., "A structure-based model for the rapid distortion of homogeneous turbulence." Technical Report TF-61, Mechanical Engineering Department, Stanford University, 1994.

Kassinos S. C. and Reynolds W. C., "A structure-based model with stropholysis effects." Annual Research Briefs 1998, Center for Turbulence Research, NASA/Stanford Univ, 1998.

Kassinos S. C. and Reynolds W. C., "Advances in structure-based turbulence modeling." Annual Research Briefs 1997, Center for Turbulence Research, NASA/Stanford Univ, 1997. 
Kassinos S. C., Langer C. A., Kalitzin G. and Iaccarino G., "A simplified structure-based model using standard turbulence scale equations: computation of rotating wall-bounded flows." International Journal of Heat and Fluid Flow, vol. 27, no. 4, pg. 653-660, 2006.

Kassinos, S.C., Langer, C.A., Haire, S.L., and Reynolds, W.C., “Structure-based turbulence modeling for wall-bounded flows." International Journal of Heat and Fluid Flow, vol. 21, pg. $559-605,2000$.

Kassinos, S.C., Reynolds, W.C., and Rogers, M.M., "One-point turbulence structure tensors." Journal of Fluid Mechanics, vol. 428, pg. 213-248, 2001.

Kim, H. G., Lee, C. M., Lim, H. C., and Kyong, N. H., “An experimental and numerical study on the flow over two-dimensional hills," Journal of Wind Engineering and Industrial Aerodynamics 66, 1997.

Kim, J.Y., Ghajar, A., Tang, C., and Foutch, G., "Comparison of near-wall treatment methods for high Reynolds number backward-facing step flow.”, International Journal of Computational Fluid Dynamics, 19, 7, 493-500, 2005.

Klebanoff, P. S., "Characteristics of turbulence in a boundary layer with zero pressure gradient.” NACA TM 1247, 1953.

Kundu, P.K., Fluid Mechanics. Academic Press, London, 1990.

Langer, C. A., and Reynolds, W. C., “A new algebraic structure-based turbulence model for rotating wall-bounded flows." Technical Report TF-85, Mechanical Engineering Department, Stanford University, 2003.

Launder, B.E., and Sharma, B.I., “Application of the energy-dissipation model of turbulence to the calculation of flow near a spinning disc." Letters in Heat and Mass Transfer, vol. 1, pg. 131-138, 1974.

Launder, B.E., and Spalding, D.B., "The numerical computation of turbulent flows." Computer Methods in applied Mechanics and Engineering, vol. 3, pg. 269-289, 1974. 
Lien, F.S., Kalitzin, G., and Durbin, P.A., "RANS modeling for compressible and transitional flows." Proceedings of the Summer Program 1998, Center for Turbulence Research, NASA/Stanford Univ, 1998.

Lumley, J.L., and Newman, G.R., "The return to isotropy of homogeneous turbulence.” J. Fluid Mech., vol. 82, part 1, pg. 161-178, 1977.

Menter, F. R., “Two-equation eddy-viscosity turbulence models for engineering applications.” AIAA Journal, vol. 32, no. 8, 1994.

Naot, D., Shavit, A., and Wolfshtein, M., "Interactions between components of the turbulence velocity correlation tensor due to pressure fluctuations.” Israel Journal of Technology, vol. 8, pg. 259-269, 1970.

O’Sullivan, J.P., Pecnik, R., and Iaccarino, G., "Investigating turbulence in wind flow over complex terrain.” Center for Turbulence Research, NASA/Stanford Univ, 2010.

Pirkl, L., and Bodnar, T., "Numerical simulation of turbulent flow around sinusoidal hills." Colloquium Fluid Dynamics, 2007.

Popovac, M., "Modeling and simulations of turbulence and heat transfer in wall-bounded flows.” Ph. D. Thesis, Delft University of Technology, 2006.

Popovac, M., "Robust eddy viscosity turbulence modeling with elliptic relaxation for external building flow analysis.” Fourth National Conference IBSPSA-USA, 2010.

Popovac, M., Hanjalic, K., “Compound wall treatment for RANS computation of complex turbulent flows and heat transfer." Flow Turbulence Combust, vol 78, pg. 177-202, 2007.

Press, William H. Numerical Recipes in C: the Art of Scientific Computing. 2nd ed. Cambridge, Cambridgeshire, Cambridge UP, pg. 362-393, 1992.

Radhakrishnan H., Pecnik R., G., Iaccarino G. and Kassinos S. C., "Computation of separated turbulent flows using the ASBM model." Proceedings of the Summer Program 2008, Center for Turbulence Research, NASA/Stanford Univ, 2008. 
Reynolds, W.C., Langer, C.A., Kassinos, S.C., " Structure and scales in turbulence modeling." Physics of Fluids, 14, 7, 2002.

Spalart, P.R., and Allmaras, S.R., “A one-equation turbulence model for aerodynamic flows.” AIAA Journal 1992-0439, 1992.

Speziale, C., Sarkar, S., and Gatski, T., "Modeling the pressure-strain correlation of turbulence: an invariant system dynamic approach.” Journal of Fluid Mechanics, vol. 227, pg. 245-272, 1991.

Storm, T.M., "Assessing the $v^{2}-f$ turbulence models for circulation control applications." Masters thesis, California Polytechnic State University San Luis Obispo, 2010.

Tannehill, John C., Dale A. Anderson, and Richard H. Pletcher. Computational Fluid Mechanics and Heat Transfer. 2nd ed. Washington, DC: Taylor \& Francis, 1997.

White, Frank M., Viscous Fluid Flow. $3^{\text {rd }}$ ed., Boston McGraw-Hill, 2006.

Wieghardt, K., and Tillmann, W., “On the Turbulent Friction Layer for Rising Pressure,” NACA TM 1314, 1951.

Wilcox, D.C., "Formulation of the $k$ - $\omega$ turbulence model revisited." AIAA Journal, vol. 46, no. 11, pg. 2823-2838, 2008.

Wilcox, D.C., "Reassessment of the scale-determining equation for advanced turbulence models.” AIAA Journal, vol. 26, no. 11, pg. 1299-1310, 1988

Wilcox, D.C., Turbulence Modeling for CFD, $3^{\text {rd }}$ ed., DCW Industries, California, 2006.

Wong, Y.S., and Li, G., "Exact finite difference schemes for solving Helmholtz equation at any wavenumber." International Journal of Numerical Analysis and Modeling Series B, vol. 2, no. 1, pg. 91-108, 2011. 